\title{
Pyrrolopyrimidine vs imidazole-phenyl- thiazole scaffolds in nonpeptidic dimerization inhibitors of Leishmania infantum trypanothione reductase
}

\section{Supporting Information}

Alejandro Revuelto, Marta Ruiz-Santaquiteria, Héctor de Lucio, Ana Gamo, Alejandra A. Carriles, Kilian Jesús Gutiérrez, Pedro A. Sánchez-Murcia, Juan A. Hermoso, Federico Gago, María-José Camarasa, Antonio Jiménez-Ruiz, and Sonsoles Velázquez*

\section{Table of contents}

\section{Chemistry}

Synthesis of intermediates $\mathbf{1 2 a}$-g for pyrrolopyrimidines $\mathbf{2 a - i}$ S2-S6

Synthesis of intermediates 18-20, 24, 32-35, 36a-f and 37e,f for

imidazole-phenyl-thiazole 3a-f

Extra references.

Table S1. Experimental water solubility and other physicochemical parameters of imidazole-phenyl-thiazole compounds 3a-f

Figure S1. Absorption and emission spectra of compound 3d.

Protein X-ray crystal structures

Table S2. Crystallographic data for Li-TryR:2f complex

Figure S2. Electron density for $\mathbf{2 f}$ ligand. S20

Figure S3. Structural changes in $L i$-TryR upon $\mathbf{2 f}$ binding. S20

Figure S4. Comparison of active site occupancy in $L i$-TryR by $\mathbf{2 f}$ and other described $T b$-TryR inhibitors

\section{Computational studies}

Figure S5. Pharmacophore model for the series 2 compounds S22

Figure S6. Calculated binding energies $\left(\mathrm{kcal} \mathrm{mol}^{-1}\right)$ per residue and component contributions for the binding of $\mathbf{3 f}$ and peptide $\mathbf{1 a}$ to $L i$-TryR monomer. S23 
Extra references

${ }^{1} \mathrm{H}$ and ${ }^{13} \mathrm{C}$ NMR spectra of selected compounds.. 


\section{Synthesis of intermediates for the preparation of pyrrolopyrimidines $2 \mathrm{a}-\mathrm{h}$}

\section{General procedure for the synthesis of 2-alkylamine-4,6-dichloropyrimidine-5-}

carbaldehydes and 4-alkylamine-2,6-dichloropyrimidine-5-carbaldehyde intermediates (12a-g and 13a-g). To a solution of commercially available 2,4,6-trichloropyrimidine-5carbaldehyde (1 g, $4.73 \mathrm{mmol})$ in dry DCM $(20 \mathrm{~mL})$ at $-78{ }^{\circ} \mathrm{C}$ under an Argon atmosphere, a solution of the appropriate amine $(4.29 \mathrm{mmol})$ and DIEA $(8.6 \mathrm{~mL})$ in dry DCM $(8 \mathrm{~mL})$ was slowly added. The reaction mixture was stirred at $-78{ }^{\circ} \mathrm{C}$ for $3 \mathrm{~h}$ and washed with water $(2 \times 20 \mathrm{~mL})$ and brine $(20 \mathrm{~mL})$. The organic layer was dried $\left(\mathrm{Na}_{2} \mathrm{SO}_{4}\right)$, filtered and evaporated to dryness. The regioisomeric crude mixture of 2-alkyl-4,6-dichloropyrimidine5-carbaldehyde 12a-g and 4-alkyl-2,6-dichloropyrimidine-5-carbaldehyde 13a-g was separated by flash chromatography performed in a Biotage ${ }^{\circledR}$ (hexane:ethylacetate, 9:1).

2-(sec-Butylamino)-4,6-dichloropyrimidine-5-carbaldehyde and 4-(sec-butylamino)2,6-dichloropyrimidine-5-carbaldehyde (12a and 13a) Following the general procedure 2,4,6-trichloropyrimidine-5-carbaldehyde was reacted with sec-butylamine $(0.43 \mathrm{~mL}, 4.29$ mmol). The final crude was purified by Biotage ${ }^{\circledR}$. The fastest moving fractions afforded $155 \mathrm{mg}(15 \%)$ of the minor regioisomer compound $\mathbf{1 2 a}$ as a yellow solid. TLC Rf (hexane:ethylacetate, 95:5): 0.14; HPLC-MS (MS, positive) (2\% to $95 \%$ of acetonitrile in 15 min $):$ Rt $=9.40 \min , m / z 248.2\left[\mathrm{M}\left({ }^{35} \mathrm{Cl}^{35} \mathrm{Cl}\right)+\mathrm{H}\right]^{+}, 250.3\left[\mathrm{M}\left({ }^{35} \mathrm{Cl}^{37} \mathrm{Cl}\right)+\mathrm{H}\right]^{+}, 252.1$ $\left[\mathrm{M}\left({ }^{37} \mathrm{Cl}^{37} \mathrm{Cl}\right)+\mathrm{H}\right]^{+} ;{ }^{1} \mathrm{H}$ NMR $\left(400 \mathrm{MHz}, \mathrm{CDCl}_{3}\right) \delta(\mathrm{ppm}): 10.25$ (s, 1H, CHO), 5.67 (bs, $1 \mathrm{H}, \mathrm{NH}), 4.11(\mathrm{~m}, 1 \mathrm{H}, \mathrm{CH}), 1.48-1.69\left(\mathrm{~m}, 2 \mathrm{H}, \mathrm{CH}_{2}\right), 1.24\left(\mathrm{~d}, J=6.6 \mathrm{~Hz}, 3 \mathrm{H}, \mathrm{CH}_{3} \mathrm{CH}\right)$, $0.96\left(\mathrm{t}, J=7.4 \mathrm{~Hz}, 3 \mathrm{H}, \mathrm{CH}_{3} \mathrm{CH}_{2}\right) ;{ }^{13} \mathrm{C} \mathrm{NMR}\left(100 \mathrm{MHz}, \mathrm{CDCl}_{3}\right) \delta(\mathrm{ppm}): 185.1(\mathrm{CHO})$, 165.2, 163.8, $160.0\left(3 \mathrm{C}_{\mathrm{Ar}}\right), 114.1\left(\underline{\mathrm{C}}_{\mathrm{Ar}} \mathrm{CHO}\right), 49.5\left(\mathrm{CH}_{2}\right), 29.5(\mathrm{CH}), 20.2\left(\mathrm{CH}_{3}\right), 10.3$ $\left(\mathrm{CH}_{3}\right)$.

The slowest moving fractions gave $860 \mathrm{mg}(81 \%)$ of the major regioisomer compound 13a as a colorless oil. TLC Rf (hexane: ethylacetate, 95:5): 0.39; HPLC-MS (ES, positive) 
( $2 \%$ to $95 \%$ of acetonitrile in $15 \mathrm{~min}$ ): $\left.\mathrm{Rt}=10.20 \mathrm{~min}, \mathrm{~m} / z 248.2\left[\mathrm{M}^{35} \mathrm{Cl}^{35} \mathrm{Cl}\right)+\mathrm{H}\right]^{+}, 250.3$ $\left[\mathrm{M}\left({ }^{35} \mathrm{Cl}^{37} \mathrm{Cl}\right)+\mathrm{H}\right]^{+}, 252.2\left[\mathrm{M}\left({ }^{37} \mathrm{Cl}^{37} \mathrm{Cl}\right)+\mathrm{H}\right]^{+} ;{ }^{1} \mathrm{H} \mathrm{NMR}\left(400 \mathrm{MHz}, \mathrm{CDCl}_{3}\right) \delta(\mathrm{ppm}): 10.29$ (s, 1H, CHO), $9.20(\mathrm{bs}, 1 \mathrm{H}, \mathrm{NH}), 4.30(\mathrm{~m}, 1 \mathrm{H}, \mathrm{CH}), 1.60\left(\mathrm{dq}, J=7.4,6.4 \mathrm{~Hz}, 2 \mathrm{H}, \mathrm{CH}_{2}\right)$, $1.23\left(\mathrm{~d}, J=6.6 \mathrm{~Hz}, 3 \mathrm{H}, \mathrm{CH}_{3} \mathrm{CH}\right), 0.93\left(\mathrm{t}, J=7.5 \mathrm{~Hz}, 3 \mathrm{H}, \mathrm{C}_{3} \mathrm{CH}_{2}\right) ;{ }^{13} \mathrm{C} \mathrm{NMR}(100 \mathrm{MHz}$, $\left.\mathrm{CDCl}_{3}\right) \delta(\mathrm{ppm}): 190.7(\mathrm{CHO}), 166.3,162.8,161.2\left(3 \mathrm{C}_{\mathrm{Ar}}\right), 106.4\left(\underline{\mathrm{C}}_{\mathrm{Ar}} \mathrm{CHO}\right), 48.9\left(\mathrm{CH}_{2}\right)$, $29.4(\mathrm{CH}), 20.1\left(\mathrm{CH}_{3}\right), 10.3\left(\mathrm{CH}_{3}\right)$.

4,6-Dichloro-2-(isobutylamino)pyrimidine-5-carbaldehyde and 2,4-dichloro-6(isobutylamino)pyrimidine-5-carbaldehyde (12b and 13b). 4,6-trichloropyrimidine-5carbaldehyde was treated with isobutylamine $(0.43 \mathrm{~mL}, 4.29 \mathrm{mmol})$ according to the general procedure. The fastest moving fractions afforded $147 \mathrm{mg}(14 \%)$ of the minor regioisomer compound $\mathbf{1 2 b}$ as a yellow solid. TLC Rf (hexane: ethylacetate, 95:5): 0.12; HPLC-MS (ES, positive) ( $2 \%$ to $95 \%$ of acetonitrile in $15 \mathrm{~min}): \mathrm{Rt}=9.47 \mathrm{~min}, \mathrm{~m} / z 248.2$ $\left[\mathrm{M}\left({ }^{35} \mathrm{Cl}^{35} \mathrm{Cl}\right)+\mathrm{H}\right]^{+}, 250.2\left[\mathrm{M}\left({ }^{35} \mathrm{Cl}^{37} \mathrm{Cl}\right)+\mathrm{H}\right]^{+}, 252.2\left[\mathrm{M}\left({ }^{37} \mathrm{Cl}^{37} \mathrm{Cl}\right)+\mathrm{H}\right]^{+} ;{ }^{1} \mathrm{H} \mathrm{NMR}(400 \mathrm{MHz}$, $\left.\mathrm{CDCl}_{3}\right) \delta(\mathrm{ppm}): 10.22(\mathrm{~s}, 1 \mathrm{H}, \mathrm{CHO}), 6.17(\mathrm{bs}, 1 \mathrm{H}, \mathrm{NH}), 3.34(\mathrm{dd}, J=6.2,6.8 \mathrm{~Hz}, 2 \mathrm{H}$, $\left.\mathrm{CH}_{2}\right), 1.90(\mathrm{~m}, 1 \mathrm{H}, \mathrm{CH}), 0.95\left(\mathrm{~d}, J=6.7 \mathrm{~Hz}, 6 \mathrm{H}, 2 \mathrm{CH}_{3}\right) ;{ }^{13} \mathrm{C} \mathrm{NMR}\left(100 \mathrm{MHz}, \mathrm{CDCl}_{3}\right) \delta$ (ppm): $185.1(\mathrm{CHO}), 165.2,163.7,160.6\left(3 \mathrm{C}_{\mathrm{Ar}}\right), 114.1\left(\underline{\mathrm{C}}_{\mathrm{Ar}} \mathrm{CHO}\right), 49.3\left(\mathrm{CH}_{2}\right), 28.4(\mathrm{CH})$, $20.1\left(2 \mathrm{CH}_{3}\right)$.

The slowest moving fractions gave $820 \mathrm{mg}(77 \%)$ of the major regioisomer compound $\mathbf{1 3 b}$ as a colorless oil. TLC Rf (hexane/ethylacetate, 95:5): 0.30. HPLC-MS (ES, positive) (2\% to $95 \%$ of acetonitrile in $15 \mathrm{~min}): \mathrm{Rt}=10.18 \mathrm{~min}, \mathrm{~m} / z 248.2\left[\mathrm{M}\left({ }^{35} \mathrm{Cl}^{35} \mathrm{Cl}\right)+\mathrm{H}\right]^{+}, 250.3$ $\left[\mathrm{M}\left({ }^{35} \mathrm{Cl}^{37} \mathrm{Cl}\right)+\mathrm{H}\right]^{+}, 252.2\left[\mathrm{M}\left({ }^{37} \mathrm{Cl}^{37} \mathrm{Cl}\right)+\mathrm{H}\right]^{+} ;{ }^{1} \mathrm{H}$ NMR $\left(400 \mathrm{MHz}, \mathrm{CDCl}_{3}\right) \delta(\mathrm{ppm}): 10.31$ (s, 1H, CHO), $9.40(\mathrm{bs}, 1 \mathrm{H}, \mathrm{NH}), 3.42\left(\mathrm{dd}, J=6.8,5.8 \mathrm{~Hz}, 2 \mathrm{H}, \mathrm{CH}_{2}\right), 1.94(\mathrm{~m}, 1 \mathrm{H}, \mathrm{CH})$, $0.98\left(\mathrm{~d}, J=6.7 \mathrm{~Hz}, 6 \mathrm{H}, 2 \mathrm{CH}_{3}\right) ;{ }^{13} \mathrm{C} \mathrm{NMR}\left(100 \mathrm{MHz}, \mathrm{CDCl}_{3}\right) \delta(\mathrm{ppm}): 190.8(\mathrm{CHO})$, 166.2, 162.9, $162.0\left(3 \mathrm{C}_{\mathrm{Ar}}\right), 106.6\left(\mathrm{C}_{\mathrm{Ar}}-\mathrm{CHO}\right), 48.8\left(\mathrm{CH}_{2}\right), 28.3(\mathrm{CH}), 20.2\left(2 \mathrm{CH}_{3}\right)$. 
dichloropyrimidine-5-carbaldehyde (12c and 13c). Following the general procedure 2,4,6trichloropyrimidine-5-carbaldehyde was reacted with benzylamine $(0.43 \mathrm{~mL}, 4.29 \mathrm{mmol})$. The fastest moving fractions afforded $186 \mathrm{mg}(15 \%)$ of the minor regioisomer compound 12c as a yellow solid. TLC Rf (hexane:ethyl acetate, 95:5): 0.18. HPLC-MS (ES, positive) ( $2 \%$ to $95 \%$ of acetonitrile in $15 \mathrm{~min}): \mathrm{Rt}=9.43 \mathrm{~min}, \mathrm{~m} / z 282.2\left[\mathrm{M}\left({ }^{35} \mathrm{Cl}^{35} \mathrm{Cl}\right)+\mathrm{H}\right]^{+}, 284.2$ $\left[\mathrm{M}\left({ }^{35} \mathrm{Cl}^{37} \mathrm{Cl}\right)+\mathrm{H}\right]^{+}, 286.3\left[\mathrm{M}\left({ }^{37} \mathrm{Cl}^{37} \mathrm{Cl}\right)+\mathrm{H}\right]^{+} ;{ }^{1} \mathrm{H} \mathrm{NMR}\left(400 \mathrm{MHz}, \mathrm{CDCl}_{3}\right) \delta(\mathrm{ppm}): 10.25$ (s, 1H, CHO), 7.26-7.40 (m, 5H, Ar), $6.42(\mathrm{bs}, 1 \mathrm{H}, \mathrm{NH}), 4.72\left(\mathrm{~d}, J=6.0 \mathrm{~Hz}, 2 \mathrm{H}, \mathrm{CH}_{2}\right) ;{ }^{13} \mathrm{C}$ NMR (100 MHz, $\left.\mathrm{CDCl}_{3}\right) \delta(\mathrm{ppm}): 185.0(\mathrm{CHO}), 165.3,163.9,160.2,\left(3 \mathrm{C}_{\mathrm{Ar}}\right), 136.7\left(\mathrm{C}_{\mathrm{Ar}}\right)$, $129.1\left(2 \mathrm{CH}_{\mathrm{Ar}}\right), 128.2\left(\mathrm{CH}_{\mathrm{Ar}}\right), 127.9\left(2 \mathrm{CH}_{\mathrm{Ar}}\right), 114.6\left(\underline{\mathrm{C}}_{\mathrm{Ar}}-\mathrm{CHO}\right), 46.0\left(\mathrm{CH}_{2}\right)$.

The slowest moving fractions gave $833 \mathrm{mg}(69 \%)$ of the major regioisomer compound 13c as a colorless oil. TLC Rf (hexane:ethyl acetate, 95:5): 0.34. HPLC-MS (ES, positive) (2\% to $95 \%$ of acetonitrile in $15 \mathrm{~min}): \mathrm{Rt}=9.98 \mathrm{~min}, \mathrm{~m} / z 282.3\left[\mathrm{M}\left({ }^{35} \mathrm{Cl}^{35} \mathrm{Cl}\right)+\mathrm{H}\right]^{+}, 284.2$ $\left[\mathrm{M}\left({ }^{35} \mathrm{Cl}^{37} \mathrm{Cl}\right)+\mathrm{H}\right]^{+}, 286.1\left[\mathrm{M}\left({ }^{37} \mathrm{Cl}^{37} \mathrm{Cl}\right)+\mathrm{H}\right]^{+} ;{ }^{1} \mathrm{H}$ NMR $\left(400 \mathrm{MHz}, \mathrm{CDCl}_{3}\right) \delta(\mathrm{ppm}): 10.31$ (s, 1H, CHO), $9.60(\mathrm{bs}, 1 \mathrm{H}, \mathrm{NH}), 7.28-7.40(\mathrm{~m}, 5 \mathrm{H}, \mathrm{Ar}), 4.78\left(\mathrm{~d}, J=5.7 \mathrm{~Hz}, 2 \mathrm{H}, \mathrm{CH}_{2}\right) ;{ }^{13} \mathrm{C}$ NMR (100 MHz, $\left.\mathrm{CDCl}_{3}\right) \delta(\mathrm{ppm}): 190.6(\mathrm{CHO}), 166.3\left(3 \mathrm{C}_{\mathrm{Ar}}\right), 163.0,161.7,136.5\left(\mathrm{C}_{\mathrm{Ar}}\right)$, $129.1\left(2 \mathrm{CH}_{\mathrm{Ar}}\right), 128.2\left(\mathrm{CH}_{\mathrm{Ar}}\right), 128.0\left(2 \mathrm{CH}_{\mathrm{Ar}}\right), 106.9\left(\mathrm{C}_{\mathrm{Ar}}-\mathrm{CHO}\right), 45.4\left(\mathrm{CH}_{2}\right)$.

4,6-Dichloropyrimidine-2-(phenylethylamino)-5-carbaldehyde 2,4dichloropyrimidine-6-(phenylethylamino)-5-carbaldehyde (12d and 13d). According to the general procedure 2,4,6-trichloropyrimidine-5-carbaldehyde was treated with phenylethylamine $(0.46 \mathrm{~mL}, 4.29 \mathrm{mmol})$. The fastest moving fractions afforded $245 \mathrm{mg}$ $(20 \%)$ of the minor regioisomer compound $\mathbf{1 2 d}$ as a yellow solid. Mp: $141-143{ }^{\circ} \mathrm{C} ;{ }^{1} \mathrm{H}$ $\operatorname{NMR}\left(400 \mathrm{MHz}, \mathrm{CDCl}_{3}\right) \delta(\mathrm{ppm}): 10.17(\mathrm{~s}, 1 \mathrm{H}, \mathrm{CHO}), 7.26-7.13\left(\mathrm{~m}, 5 \mathrm{H}, 5 \mathrm{CH}_{\mathrm{Ar}}\right), 6.10(\mathrm{~s}$, $1 \mathrm{H}, \mathrm{NH}), 3.73\left(\mathrm{q}, J=6.8 \mathrm{~Hz}, 2 \mathrm{H}, \mathrm{CH}_{2}-\mathrm{NH}\right), 2.86\left(\mathrm{t}, J=6.9 \mathrm{~Hz}, 2 \mathrm{H}, \mathrm{CH}_{2}-\mathrm{Ph}\right) ;{ }^{13} \mathrm{C} \mathrm{NMR}$ 
$\left(100 \mathrm{MHz}, \mathrm{CDCl}_{3}\right) \delta(\mathrm{ppm}): 183.8(\mathrm{CHO}), 164.1\left(\mathrm{C}_{\mathrm{pir}}\right), 162.6\left(\mathrm{C}_{\mathrm{pir}}\right), 159.2\left(\mathrm{C}_{\mathrm{pir}}\right), 136.8$ $\left(\mathrm{C}_{\mathrm{Ar}}\right), 127.7\left(4 \mathrm{CH}_{\mathrm{Ar}}\right), 125.9\left(\mathrm{CH}_{\mathrm{Ar}}\right), 113.2\left(\mathrm{C}_{\mathrm{pir}}-\mathrm{CHO}\right), 42.0\left(\mathrm{C}_{2}-\mathrm{NH}\right), 34.2\left(\mathrm{C}_{2}-\mathrm{Ph}\right)$.

The slowest moving fractions gave $650 \mathrm{mg}(52 \%)$ of the major regioisomer compound 13d as a yellow solid. Mp: $116-118{ }^{\circ} \mathrm{C} ;{ }^{1} \mathrm{H}$ NMR (400 MHz, $\left.\mathrm{CDCl}_{3}\right) \delta(\mathrm{ppm}): 10.26(\mathrm{~s}, 1 \mathrm{H}$, CHO), 9.32 (bs, 1H, NH), 7.20-7.36 (m, 5H, 5CHAr), 3.84 (q, J = 7.0 Hz, 2H, $\mathrm{CH}_{2}-\mathrm{NH}$ ), $2.94\left(\mathrm{t}, J=7.1 \mathrm{~Hz}, 2 \mathrm{H}, \mathrm{C}_{2}-\mathrm{Ph}\right) ;{ }^{13} \mathrm{C} \mathrm{NMR}\left(100 \mathrm{MHz}, \mathrm{CDCl}_{3}\right) \delta(\mathrm{ppm}): 190.4(\mathrm{CHO})$, $166.0\left(\mathrm{C}_{\text {pir }}\right), 162.8\left(\mathrm{C}_{\text {pir }}\right), 161.7\left(\mathrm{C}_{\text {pir }}\right), 137.9\left(\mathrm{C}_{\mathrm{Ar}}\right), 128.8\left(4 \mathrm{CH}_{\mathrm{Ar}}\right), 126.9\left(\mathrm{CH}_{\mathrm{Ar}}\right), 106.7$ $\left(\mathrm{C}_{\mathrm{pir}}-\mathrm{CHO}\right), 42.8\left(\mathrm{C}_{2}-\mathrm{NH}\right), 35.2\left(\mathrm{C}_{2}-\mathrm{Ph}\right)$.

4,6-Dichloro-2-(3-phenylpropylamino)pyrimidine-5-carbaldehyde and 2,4-dichloro-6-(3phenylpropylamino)pyrimidine-5-carbaldehyde (12e and 13e). 2,4,6-trichloropyrimidine5-carbaldehyde was treated with phenylpropylamine $(0.46 \mathrm{~mL}, 4.29 \mathrm{mmol})$ according to the general procedure. The fastest moving fractions afforded $183 \mathrm{mg}(12 \%)$ of the minor regioisomer compound 12e as a yellow solid. Mp: 107-109 ${ }^{\circ} \mathrm{C} ;{ }^{1} \mathrm{H}$ NMR (400 MHz, $\left.\mathrm{CDCl}_{3}\right) \delta(\mathrm{ppm}): 10.18(\mathrm{~s}, 1 \mathrm{H}, \mathrm{CHO}), 7.24-7.10\left(\mathrm{~m}, 5 \mathrm{H}, 5 \mathrm{CH}_{\mathrm{Ar}}\right), 5.99(\mathrm{bs}, 1 \mathrm{H}, \mathrm{NH}), 3.50-$ $3.45\left(\mathrm{q}, J=6.8 \mathrm{~Hz}, 2 \mathrm{H}, \mathrm{CH}_{2}-\mathrm{NH}\right), 2.64\left(\mathrm{t}, J=7.6 \mathrm{~Hz}, 2 \mathrm{H}, \mathrm{CH}_{2}-\mathrm{Ph}\right), 1.94-1.87(\mathrm{~m}, 2 \mathrm{H}$, $\left.\mathrm{CH}_{2}-\mathrm{CH}_{2}-\mathrm{CH}_{2}\right) ;{ }^{13} \mathrm{C} \mathrm{NMR}\left(100 \mathrm{MHz}, \mathrm{CDCl}_{3}\right) \delta(\mathrm{ppm}): 183.9(\mathrm{CHO}), 164.1\left(\mathrm{C}_{\mathrm{pir}}-\mathrm{NH}\right)$, $162.6\left(\mathrm{C}_{\mathrm{pir}}-\mathrm{Cl}\right), 159.3\left(\mathrm{C}_{\mathrm{pir}}-\mathrm{Cl}\right), 139.7\left(\mathrm{C}_{\mathrm{Ar}}\right), 127.5\left(2 \mathrm{CH}_{\mathrm{Ar}}\right), 127.3\left(2 \mathrm{CH}_{\mathrm{Ar}}\right), 125.2\left(\mathrm{CH}_{\mathrm{Ar}}\right)$, $113.1\left(\mathrm{C}_{\text {pir }}-\mathrm{CHO}\right), 40.41\left(\mathrm{CH}_{2}-\mathrm{NH}\right), 31.9\left(\mathrm{CH}_{2}-\mathrm{Ph}\right), 29.6\left(\mathrm{CH}_{2}-\underline{\mathrm{CH}_{2}}-\mathrm{CH}_{2}\right)$.

The slowest moving fractions gave $890 \mathrm{mg}(67 \%)$ of the major regioisomer compound $13 \mathbf{e}$ as a white solid. Mp: 89-91 ${ }^{\circ} \mathrm{C} ;{ }^{1} \mathrm{H}$ NMR $\left(400 \mathrm{MHz}, \mathrm{CDCl}_{3}\right) \delta(\mathrm{ppm}): 10.22$ (s, 1H, CHO), $9.27(\mathrm{~s}, 1 \mathrm{H}, \mathrm{NH}), 7.24-7.11\left(\mathrm{~m}, 5 \mathrm{H}, 5 \mathrm{CH}_{\mathrm{Ar}}\right), 3.53\left(\mathrm{q}, J=6.9 \mathrm{~Hz}, 2 \mathrm{H}, \mathrm{CH}_{2}-\mathrm{NH}\right), 2.64(\mathrm{t}, J=$ $\left.7.5 \mathrm{~Hz}, 2 \mathrm{H}, \mathrm{CH}_{2}-\mathrm{Ph}\right), 1.97-1.88\left(\mathrm{~m}, 2 \mathrm{H}, \mathrm{CH}_{2}-\mathrm{CH}_{2}-\mathrm{CH}_{2}\right) ;{ }^{13} \mathrm{C} \mathrm{NMR}\left(100 \mathrm{MHz}, \mathrm{CDCl}_{3}\right) \delta$ (ppm): $189.5(\mathrm{CHO}), 165.0\left(\mathrm{C}_{\mathrm{pir}}-\mathrm{NH}\right), 161.8\left(\mathrm{C}_{\mathrm{pir}}-\mathrm{Cl}\right), 160.7\left(\mathrm{C}_{\mathrm{pir}}-\mathrm{Cl}\right), 139.6\left(\mathrm{C}_{\mathrm{Ar}}\right), 127.50$ $\left(2 \mathrm{CH}_{\mathrm{Ar}}\right), 127.3\left(2 \mathrm{CH}_{\mathrm{Ar}}\right), 125.2\left(\mathrm{CH}_{\mathrm{Ar}}\right), 105.6\left(\mathrm{C}_{\mathrm{pir}}-\mathrm{CHO}\right), 39.8\left(\underline{\mathrm{CH}}_{2}-\mathrm{NH}\right), 31.9\left(\underline{\mathrm{CH}}_{2}-\mathrm{Ph}\right)$, $29.3\left(\mathrm{CH}_{2}-\mathrm{CH}_{2}-\mathrm{CH}_{2}\right)$. 
4,6-Dichloro-2-[(2-naphthalen-2-yl)ethylamino]pyrimidine-5-carbaldehyde and 2,4dichloro-6-[(2-naphthalen-2-yl)ethylamino]pyrimidine-5-carbaldehyde (12f and 13f). Following the general procedure 2,4,6-trichloropyrimidine-5-carbaldehyde was reacted with 2-naphthylethylamine $(0.46 \mathrm{~mL}, 4.29 \mathrm{mmol})$. The fastest moving fractions afforded $142 \mathrm{mg}(10 \%)$ of the desired compound $\mathbf{1 2 f}$ as a yellow solid. Mp: 194-196 ${ }^{\circ} \mathrm{C} ;{ }^{1} \mathrm{H}$ NMR (400 MHz, $\left.\mathrm{CDCl}_{3}\right) \delta(\mathrm{ppm}): 10.03(\mathrm{~s}, 1 \mathrm{H}, \mathrm{CHO}), 9.21(\mathrm{t}, J=5.6 \mathrm{~Hz}, 1 \mathrm{H}, \mathrm{NH}), 7.89-7.82$ $\left(\mathrm{m}, 3 \mathrm{H}, 3 \mathrm{CH}_{\mathrm{Ar}}\right), 7.74\left(\mathrm{~s}, 1 \mathrm{H}, \mathrm{CH}_{\mathrm{Ar}}\right), 7.50-7.40\left(\mathrm{~m}, 3 \mathrm{H}, 3 \mathrm{CH}_{\mathrm{Ar}}\right), 3.68(\mathrm{q}, J=6.7 \mathrm{~Hz}, 2 \mathrm{H}$, $\left.\mathrm{C}_{2}-\mathrm{NH}\right), 3.03$ (t, $J=7.1 \mathrm{~Hz}, 2 \mathrm{H}, \underline{\mathrm{CH}}_{2}-$ naphthyl); ${ }^{13} \mathrm{C} \mathrm{NMR}\left(100 \mathrm{MHz}, \mathrm{CDCl}_{3}\right) \delta(\mathrm{ppm})$ : $185.1(\mathrm{CHO}), 163.9\left(\mathrm{C}_{\mathrm{pir}}-\mathrm{Cl}\right), 162.8\left(\mathrm{C}_{\mathrm{pir}}-\mathrm{Cl}\right), 160.3\left(\mathrm{C}_{\mathrm{pir}}-\mathrm{NH}\right), 136.9\left(\mathrm{C}_{\mathrm{Ar}}\right), 133.5\left(\mathrm{C}_{\mathrm{Ar}}\right)$, $132.2\left(\mathrm{C}_{\mathrm{Ar}}\right), 128.3,127.9,127.9,127.8,127.3,126.5,125.9,113.1\left(\mathrm{C}_{\mathrm{pir}}-\mathrm{CHO}\right), 43.0\left(\underline{\mathrm{C}}_{2^{-}}\right.$ $\mathrm{NH}), 34.8$ ( $\mathrm{CH}_{2}$-naphthyl).

The slowest moving fractions gave $493 \mathrm{mg}(33 \%)$ of the major regioisomer compound $\mathbf{1 3 f}$ as a white solid. Mp: $126-128{ }^{\circ} \mathrm{C} ;{ }^{1} \mathrm{H}$ NMR $\left(400 \mathrm{MHz}, \mathrm{CDCl}_{3}\right) \delta(\mathrm{ppm}): 10.23(\mathrm{~s}, 1 \mathrm{H}$, CHO), $9.36(\mathrm{~s}, 1 \mathrm{H}, \mathrm{NH}), 7.83-7.79\left(\mathrm{~m}, 3 \mathrm{H}_{\mathrm{Ar}}\right), 7.68\left(\mathrm{~s}, 1 \mathrm{H}_{\mathrm{Ar}}\right), 7.51-7.42\left(\mathrm{~m}, 2 \mathrm{H}_{\mathrm{Ar}}\right), 7.36$ $\left(\mathrm{dd}, J=8.4,1.5 \mathrm{~Hz}, 1 \mathrm{H}_{\mathrm{Ar}}\right), 3.92\left(\mathrm{q}, \mathrm{J}=7.0 \mathrm{~Hz}, 2 \mathrm{H}, \underline{\mathrm{CH}}_{2}-\mathrm{NH}\right), 3.10\left(\mathrm{t}, J=7.1 \mathrm{~Hz}, 2 \mathrm{H}, \mathrm{CH}_{\underline{2}}-\right.$ naphthyl); ${ }^{13} \mathrm{C}$ NMR (100 MHz, $\left.\mathrm{CDCl}_{3}\right) \delta(\mathrm{ppm}): 189.3(\mathrm{CHO}), 164.9\left(\mathrm{C}_{\mathrm{pir}}-\mathrm{Cl}\right), 161.7$ $\left(\mathrm{C}_{\text {pir }}-\mathrm{NH}\right), 160.6\left(\mathrm{C}_{\mathrm{pir}}-\mathrm{Cl}\right), 134.3\left(\mathrm{C}_{\mathrm{Ar}}\right), 132.5\left(\mathrm{C}_{\mathrm{Ar}}\right), 131.3\left(\mathrm{C}_{\mathrm{Ar}}\right), 127.5,126.6,126.5,126.3$, $125.9,125.2,124.6,105.6\left(\underline{\mathrm{C}}_{\mathrm{pir}}-\mathrm{CHO}\right), 41.6\left(\underline{\mathrm{CH}}_{2}-\mathrm{NH}\right), 34.3\left(\underline{\mathrm{CH}}_{2}-\right.$ naphthyl).

2-(([1,1'-Biphenyl]-4-yl)methylamino)-4,6-dichloropyrimidine-5-carbaldehyde and 4(([1,1'-Biphenyl]-4-yl)methylamino)-2,6-dichloropyrimidine-5-carbaldehyde $\mathbf{( 1 2 g}$ and 13g). 2,4,6-trichloropyrimidine-5-carbaldehyde was treated with 2-biphenylmethylamine (0.46 $\mathrm{mL}, 4.29 \mathrm{mmol})$ according to the general procedure. The fastest moving fractions afforded $188 \mathrm{mg}(12 \%)$ of the minor regioisomer compound $\mathbf{1 2 g}$ as a yellow solid. ${ }^{1} \mathrm{H}$ NMR (400 MHz, $\left.\mathrm{CDCl}_{3}\right) \delta: 10.25(\mathrm{~s}, 1 \mathrm{H}, \mathrm{CHO}), 9.52(\mathrm{~s}, 1 \mathrm{H}, \mathrm{NH}), 7.52-7.46\left(\mathrm{~m}, 4 \mathrm{H}_{\mathrm{Ar}}\right)$, $7.37-7.23\left(\mathrm{~m}, 5 \mathrm{H}_{\mathrm{Ar}}\right), 4.65\left(\mathrm{~d}, J=5.7 \mathrm{~Hz}, 2 \mathrm{H}, \mathrm{CH}_{2}\right)$; 
The slowest moving fractions gave $693 \mathrm{mg}(45 \%)$ of the major regioisomer compound $\mathbf{1 3 g}$ as a white solid. ${ }^{1} \mathrm{H}$ NMR (400 MHz, $\left.\mathrm{CDCl}_{3}\right) \delta(\mathrm{ppm}): 10.18(\mathrm{~s}, 1 \mathrm{H}, \mathrm{CHO}), 9.52(\mathrm{~s}, 1 \mathrm{H}$, $\mathrm{NH}), 7.50-7.44\left(\mathrm{~m}, 4 \mathrm{H}_{\mathrm{Ar}}\right), 7.35-7.20\left(\mathrm{~m}, 5 \mathrm{H}_{\mathrm{Ar}}\right), 4.69\left(\mathrm{~d}, J=5.8 \mathrm{~Hz}, 2 \mathrm{H}, \mathrm{CH}_{2}\right)$;

\section{Synthesis of intermediates for the preparation of imidazole-phenyl-thiazole 3a-f}

4-Bromo-2-hydroxybenzonitrile (19). ${ }^{1}$ To a solution of 4-bromo-2-fluoro-benzonitrile 17 (211 mg, $1.06 \mathrm{mmol})$ and 3-hydroxypropanamide (94 mg, 1.06mmol) in dry THF (20 mL) under argon atmosphere, a solution of 0.5 M KHDMS $(2.11 \mathrm{ml}, 1.06 \mathrm{mmol})$ in toluene is added at $0{ }^{\circ} \mathrm{C}$. The reaction mixture is stirred at room temperature overnight and the solvent is evaporated at dryness. The crude is dissolved in DCM $(30 \mathrm{~mL})$, washed with $\mathrm{HCl} 0.1 \mathrm{~N}(3 \times 30 \mathrm{~mL})$, dried $\left(\mathrm{Na}_{2} \mathrm{SO}_{4}\right)$, filtered and evaporated. The residue is purified by Biotage $^{\circledR}$ (from $0 \% \mathrm{MeOH}$ to $65 \% \mathrm{MeOH}$ ) to give $73 \mathrm{mg}$ (35\% yield) of 19 as a white solid. ${ }^{1} \mathrm{H}$ NMR (DMSO-d 6 , $\left.300 \mathrm{MHz}\right) \delta(\mathrm{ppm}): 11.59$ (bs, 1H, OH), 7.58 (d, $J=8.3 \mathrm{~Hz}$, 2H, Ar), 7.18 (s, 1H, Ar), 7.15 (dd, $J=8.3 \mathrm{~Hz}, J=1.0 \mathrm{~Hz}, 1 \mathrm{H}, \mathrm{Ar}$ ); MS (ESI, positive) $m / z$ : $199.0[\mathrm{M}+\mathrm{H}]^{+}$

3-(5-Bromo-2-cyanophenoxy)propanamide (18). A solution of 3-hydroxypropanamide (313 $\mathrm{mg}, 3.51 \mathrm{mmol})$ and 4-bromo-2-fluorobenzonitrilo $17(0.639 \mathrm{~g}, 3.51 \mathrm{mmol})$ in dry DMF $(30 \mathrm{~mL})$ is reacted with $\mathrm{Cs}_{2} \mathrm{CO}_{3}(1.26 \mathrm{~g}, 3.86 \mathrm{mmol})$, previously dried with $\mathrm{P}_{2} \mathrm{O}_{5}$ under vacuum, at room temperature for $24 \mathrm{~h}$. Then, the reaction mixture is filtered, washed with ethyl acetate and the filtrates are evaporated to dryness. The residue is washed with water $(2 \times 50 \mathrm{~mL})$ and brine $(2 \times 50 \mathrm{~mL})$. The organic layer is dried $\left(\mathrm{Na}_{2} \mathrm{SO}_{4}\right)$, filtered and evaporated. The final residue is purified by flash column chromatography in silica gel (DCM/MeOH, $50: 1$ to $20: 1)$ to yield $282 \mathrm{mg}$ (30\% yield) of $\mathbf{1 8}$ as a white solid. Mp: 120-126 ${ }^{\circ} \mathrm{C} ;{ }^{1} \mathrm{H}$ NMR (DMSO-d $\left.6,300 \mathrm{MHz}\right) \delta(\mathrm{ppm}): 7.68$ (d, $\left.J=6.9 \mathrm{~Hz}, 2 \mathrm{H}, \mathrm{Ar}\right), 7.54$ (s, 1H, Ar), 7.47 (bs, 1H, CONH$\left.)_{2}\right), 7.31$ (d, $\left.J=6.9 \mathrm{~Hz}, 1 \mathrm{H}, \mathrm{Ar}\right), 6.97$ (bs, 1H, $\mathrm{CONH}_{2}$ ), 
$4.36\left(\mathrm{t}, J=5.8 \mathrm{~Hz}, 2 \mathrm{H}, \mathrm{OCH}_{2}\right), 2.57\left(\mathrm{t}, J=5.8 \mathrm{~Hz}, 2 \mathrm{H}, \mathrm{OCH}_{2} \mathrm{CH}_{2}\right) ;{ }^{13} \mathrm{C}$ NMR (DMSO-d 6 , $75 \mathrm{MHz}) \delta(\mathrm{ppm}): 171.0\left(\mathrm{CONH}_{2}\right), 160.5\left(\mathrm{OC}_{\mathrm{Ar}}\right), 134.9\left(\mathrm{CH}_{\mathrm{Ar}}\right), 128.6\left(\mathrm{C}_{\mathrm{Ar}}\right), 124.2\left(\mathrm{CH}_{\mathrm{Ar}}\right)$, 116.5 $\left(\mathrm{CH}_{\mathrm{Ar}}\right), 115.7(\mathrm{CN}), 99.9\left(\mathrm{C}_{\mathrm{Ar}}\right), 65.9\left(\mathrm{OCH}_{2}\right), 34.4\left(\mathrm{OCH}_{2} \mathrm{CH}_{2}\right)$;

4-Bromo-2-(2-(2-oxoimidazolidinyl)ethoxy)benzonitrile (20). To a solution of 1-(2hydroxyethyl)imidazolidin-2-one (445 mg, $3.25 \mathrm{mmol})$ and 4-bromo-2-fluorobenzonitrile $17(650 \mathrm{mg}, 3.25 \mathrm{mmol})$ in dry DMF $(40 \mathrm{~mL})$ under an argon atmosphere, was added KHMDS $0,5 \mathrm{M}$ in toluene $(7.5 \mathrm{ml}, 3.75 \mathrm{mmol})$ at $0{ }^{\circ} \mathrm{C}$. The reaction mixture wass stirred at room temperature overnight and the solvent wass evaporated to dryness. The residue is dissolved in DCM $(50 \mathrm{~mL})$ and washed with $\mathrm{HCl}(3 \times 30 \mathrm{~mL})$. The aqueous layers are extracted with DCM $(2 \times 50 \mathrm{~mL})$ and the combined organic layers are washed with brine $(50 \mathrm{~mL})$, dried $\left(\mathrm{Na}_{2} \mathrm{SO}_{4}\right)$, filtered and evaporated to dryness. The residue is purified by flash column chromatography in silica gel (DCM/MeOH, $9.75: 0.25)$ to yield $713 \mathrm{mg}$ (71\% yield) of $\mathbf{2 0}$ as a yellow solid. Mp: $165-171{ }^{\circ} \mathrm{C}$; IR (KBr), $v(\mathrm{~cm}-1): 3250$ (NH st), $2227(\mathrm{C} \equiv \mathrm{N}), 1685(\mathrm{C}=\mathrm{O}) ;{ }^{1} \mathrm{H}$ NMR $\left(\mathrm{DMSO}_{6}, 400 \mathrm{MHz}\right) \delta(\mathrm{ppm}): 7.69(\mathrm{~d}, J=8.2 \mathrm{~Hz}$, 1H, Ar), 7.56 (d, $J=1.8 \mathrm{~Hz}, 1 \mathrm{H}, \mathrm{Ar}), 7.32(\mathrm{dd}, J=8.2 \mathrm{~Hz}, J=1.7 \mathrm{~Hz}, 1 \mathrm{H}, \mathrm{Ar}), 6.42$ (bs, $1 \mathrm{H}, \mathrm{NHCON}), 4.26\left(\mathrm{t}, J=5.2 \mathrm{~Hz}, 2 \mathrm{H}, \mathrm{OCH}_{2}\right), 3.57-3.46\left(\mathrm{~m}, 2 \mathrm{H}, \mathrm{CH}_{2} \mathrm{CH}_{2} \mathrm{NHCON}\right), 3.44$ (t, $\left.J=5.2 \mathrm{~Hz}, 2 \mathrm{H}, \mathrm{OCH}_{2} \mathrm{CH}_{2}\right), 3.23\left(\mathrm{t}, J=7.8 \mathrm{~Hz}, 2 \mathrm{H}, \mathrm{CH}_{2} \underline{\mathrm{C}}_{2} \mathrm{NHCON}^{\mathrm{N}}\right) ;{ }^{13} \mathrm{C} \mathrm{NMR}$ $\left(\mathrm{DMSO}_{6}, 75 \mathrm{MHz}\right) \delta(\mathrm{ppm}): 162.1(\mathrm{NHCON}), 160.5\left(\mathrm{OC}_{\mathrm{Ar}}\right), 134.9\left(\mathrm{CH}_{\mathrm{Ar}}\right), 128.6\left(\mathrm{C}_{\mathrm{Ar}}\right)$,

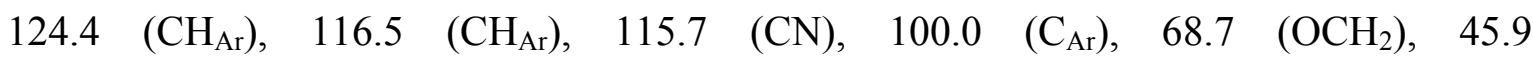
$\left(\underline{\mathrm{CH}}_{2} \mathrm{CH}_{2} \mathrm{NHCON}\right), 42.4\left(\mathrm{OCH}_{2} \underline{\mathrm{CH}}_{2}\right), 37.5\left(\mathrm{CH}_{2} \underline{\mathrm{CH}}_{2} \mathrm{NHCON}\right) ;$ MS (ESI, positive) $\mathrm{m} / z$ : $332.0[\mathrm{M}+\mathrm{Na}]^{+}, 310.0[\mathrm{M}+\mathrm{H}]^{+}$

tert-Butyl 2-((1H-imidazol-2-yl)methylamino)ethylcarbamate (24). To a solution of tertbutyl (2-aminoethyl)carbamate (1.65 g, $10.4 \mathrm{mmol})$ in $\mathrm{MeOH}$ (40 ml), 2-(1H-imidazol-2yl)acetaldehyde 23 (1.03 g, $10.4 \mathrm{mmol})$ was added and the reaction mixture is stirred at room temperature for $1 \mathrm{~h}$. Then, $\mathrm{NaBH}_{4}(0.59 \mathrm{~g}, 15.6 \mathrm{mmol})$ was added at $0{ }^{\circ} \mathrm{C}$. After $1 \mathrm{~h}$, 
the reaction was quenched with a saturated $\mathrm{NH}_{4} \mathrm{Cl}$ solution $(60 \mathrm{ml})$. The reaction mixture was then treated with a saturated $\mathrm{NaHCO}_{3}$ solution until $\mathrm{pH}=8-9$. The solvent was removed at reduced pressure and the residue was purified by Biotage ${ }^{\circledR}$ (from $0 \%$ to $60 \%$ $\mathrm{MeOH})$ to give $1.97 \mathrm{~g}$ (79\% yield) of $\mathbf{2 4}$ as a white solid. $\mathrm{Mp}: 157-159{ }^{\circ} \mathrm{C}$; IR (KBr), $v$ $\left(\mathrm{cm}^{-1}\right)$ : 3222 (NH-st), $1691(\mathrm{C}=\mathrm{O}) ;{ }^{1} \mathrm{H}$ NMR $\left(\mathrm{DMSO}_{6}, \mathrm{~d}_{6}, 300 \mathrm{MHz}\right) \delta(\mathrm{ppm}): 6.87(\mathrm{~s}, 2 \mathrm{H}$,

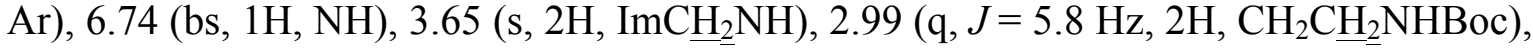

$2.52\left(\mathrm{t}, J=6.5 \mathrm{~Hz}, 2 \mathrm{H}, \mathrm{CH}_{2} \mathrm{CH}_{2} \mathrm{NHBoc}\right), 1.37$ (s, 9H, $\left.\mathrm{CH}_{3}\right) ;{ }^{13} \mathrm{C}$ NMR (DMSO-d 6 , 75 MHz) $\delta(\mathrm{ppm}): 155.6(\mathrm{NHCOO}), 140.0\left(\mathrm{C}_{\mathrm{Ar}}\right), 122.5\left(\mathrm{CH}_{\mathrm{Ar}}\right), 122.5\left(\mathrm{CH}_{\mathrm{Ar}}\right), 78.1(\mathrm{OC}), 46.3$ (ImCH $2 \mathrm{~N}), 43.1\left(\underline{\mathrm{CH}}_{2} \mathrm{CH}_{2} \mathrm{NHBoc}\right), 36.7\left(\mathrm{CH}_{2} \underline{\mathrm{CH}}_{2} \mathrm{NHBoc}\right), 28.2\left(\mathrm{CH}_{3}\right) ; \mathrm{MS}$ (ESI, positive) $m / z: 241.2[\mathrm{M}+\mathrm{H}]^{+}$

\section{Synthesis of non-commercially available $\alpha$-bromo ketones}

\section{General procedure of bromination of alkyl derivatives 28 and $29 .^{2}$}

To a solution of commercially available ketone $\mathbf{2 8}$ and $29(8.75 \mathrm{mmol})$ dissolved in methanol $(15 \mathrm{ml})$ was added dropwise $\mathrm{Br}_{2}(0.45 \mathrm{ml}, 8.75 \mathrm{mmol})$ at $0{ }^{\circ} \mathrm{C}$ with stirring. Then, the mixture was allowed to consume $\mathrm{Br}_{2}$ until disappearance of color ( $3 \mathrm{~h}$ approx) at room temperature. $30 \mathrm{ml}$ of water was added and the crude was also diluted with $30 \mathrm{ml}$ of ethyl ether. The organic layer was separated and the aqueous phase was extract with ethyl ether $(2 \times 30 \mathrm{ml})$. The organic extracts were put together, dried over $\mathrm{Na}_{2} \mathrm{SO}_{4}$ and carefully evaporated in vacuo. It was obtained pure colorless tear oils $\mathbf{3 2}$ and $\mathbf{3 3}$

1-bromo-4-methylpentan-2-one (32). Yield $68 \%{ }^{1} \mathrm{H} \mathrm{NMR}\left(\mathrm{CDCl}_{3}, 400 \mathrm{MHz}\right) \delta(\mathrm{ppm})$ : $3.85\left(\mathrm{~s}, 2 \mathrm{H}, \mathrm{CH}_{2} \mathrm{Br}\right), 2.51\left(\mathrm{~d}, J=6.9 \mathrm{~Hz}, 2 \mathrm{H}, \mathrm{CH}_{2} \mathrm{CO}\right), 2.16$ (sept, $1 \mathrm{H}, J=6.7 \mathrm{~Hz}$, $\left.\left(\mathrm{CH}_{3}\right)_{2} \mathrm{CH}\right), 0.93\left(\mathrm{~d}, J=6.7 \mathrm{~Hz}, 6 \mathrm{H}, \mathrm{CH}_{3}\right) ;{ }^{13} \mathrm{C} \mathrm{NMR}\left(\mathrm{CDCl}_{3}, 75 \mathrm{MHz}\right) \delta(\mathrm{ppm}): 201.7$ (CO), $48.8\left(\mathrm{CH}_{2} \mathrm{Br}\right), 34.8\left(\underline{\mathrm{CH}}_{2} \mathrm{CO}\right), 24.8\left(\left(\mathrm{CH}_{3}\right)_{2} \underline{\mathrm{CH}}\right), 22.5\left(\mathrm{CH}_{3}\right)$ 
1-bromo-4,4-dimethylpentan-2-one (33). Yield 52\% ${ }^{1} \mathrm{H} \mathrm{NMR}\left(\mathrm{CDCl}_{3}, 400 \mathrm{MHz}\right) \delta(\mathrm{ppm})$ : $3.86\left(\mathrm{~s}, 2 \mathrm{H}, \mathrm{CH}_{2} \mathrm{Br}\right), 2.52\left(\mathrm{~s}, 2 \mathrm{H}, \mathrm{CH}_{2} \mathrm{CO}\right), 1.03\left(\mathrm{~s}, 9 \mathrm{H}, \mathrm{CH}_{3}\right) ;{ }^{13} \mathrm{C} \mathrm{NMR}\left(\mathrm{CDCl}_{3}, 75 \mathrm{MHz}\right)$ $\delta$ (ppm): $201.1(\mathrm{CO}), 52.0\left(\mathrm{CH}_{2} \mathrm{Br}\right), 36.3\left(\mathrm{CH}_{2} \mathrm{CO}\right), 31.3\left(\left(\mathrm{CH}_{3}\right)_{3} \mathrm{C}\right), 29.6\left(\mathrm{CH}_{3}\right)$

General procedure of Claisen-Schmidt condensation (30 and 31). The commercially available corresponding arylcarboxaldehyde $(6.4 \mathrm{mmol})$ was reacted with acetone (32 mmol) in methanol $(15 \mathrm{~mL})$ in the presence of $\mathrm{NaOH} 10 \%$ aq $(32 \mathrm{mmol})$ as the base. The reaction mixture was stirred at room temperature for $2 \mathrm{~h}$ observing the appearance of a yellow precipitate. Then, the solid was filtered and recrystallize in EtOH to give intermediates $\mathbf{3 0}$ and $\mathbf{3 1}$ as yellow solids.

(E)-4-(Naphthalen-2-yl)but-3-en-2-one (30). ${ }^{3}$ Yield: 91\% M.p: $88-91{ }^{\circ} \mathrm{C} ;{ }^{1} \mathrm{H}$ NMR $\left(\mathrm{CDCl}_{3}, 400 \mathrm{MHz}\right) \delta(\mathrm{ppm}): 7.96(\mathrm{~d}, J=1.8 \mathrm{~Hz}, 1 \mathrm{H}, \mathrm{Ar}), 7.90-7.82(\mathrm{~m}, 3 \mathrm{H}, \mathrm{Ar}$, $\mathrm{C} \underline{\mathrm{H}}=\mathrm{CHCO}), 7.74-7.63(\mathrm{~m}, 2 \mathrm{H}, \mathrm{Ar}), 7.55-7.40(\mathrm{~m}, 2 \mathrm{H}, \mathrm{Ar}), 6.84(\mathrm{~d}, J=16.2 \mathrm{~Hz}, 1 \mathrm{H}$, $\mathrm{CH}=\mathrm{CHCO}), 2.43\left(\mathrm{~s}, 3 \mathrm{H}, \mathrm{CH}_{3}\right) ;{ }^{13} \mathrm{C} \mathrm{NMR}\left(\mathrm{CDCl}_{3}, 75 \mathrm{MHz}\right) \delta(\mathrm{ppm}): 198.5(\mathrm{CO}), 143.7$ $(\mathrm{Ar} \underline{\mathrm{CH}}=\mathrm{CH}), 134.5\left(\mathrm{C}_{\mathrm{Ar}}\right), 133.4\left(\mathrm{C}_{\mathrm{Ar}}\right), 132.1\left(\mathrm{C}_{\mathrm{Ar}}\right), 130.5\left(\mathrm{CH}_{\mathrm{Ar}}\right), 128.9\left(\mathrm{CH}_{\mathrm{Ar}}\right), 128.7$ $\left(\mathrm{CH}_{\mathrm{Ar}}\right), 128.0\left(\mathrm{CH}_{\mathrm{Ar}}\right), 127.5(\mathrm{ArCH}=\underline{\mathrm{CH}}), 127.4\left(\mathrm{CH}_{\mathrm{Ar}}\right), 126.9\left(\mathrm{CH}_{\mathrm{Ar}}\right), 123.6\left(\mathrm{CH}_{\mathrm{Ar}}\right), 27.8$ $\left(\mathrm{CH}_{3}\right) ; \mathrm{MS}\left(\mathrm{ESI}\right.$, positive) $\mathrm{m} / \mathrm{z}: 219.0[\mathrm{M}+\mathrm{Na}]^{+}, 197.0[\mathrm{M}+\mathrm{H}]^{+}$ (E)-4-([1,1'-Biphenyl]-4-yl)but-3-en-2-one (31).

Yield: 83\%. M.p: $129-137{ }^{\circ} \mathrm{C} ;{ }^{1} \mathrm{H}$ NMR $\left(\mathrm{CDCl}_{3}, 400 \mathrm{MHz}\right) \delta(\mathrm{ppm}): 7.67-7.60(\mathrm{~m}, 6 \mathrm{H}$, Ar), $7.56(\mathrm{~d}, J=16.3 \mathrm{~Hz}, 1 \mathrm{H}, \mathrm{CH}=\mathrm{CHCO}), 7.46(\mathrm{t}, J=7.6 \mathrm{~Hz}, 2 \mathrm{H}, \mathrm{Ar}), 7.45-7.31$ (m, $1 \mathrm{H}, \mathrm{Ar}), 6.76(\mathrm{~d}, J=16.2 \mathrm{~Hz}, 1 \mathrm{H}, \mathrm{CH}=\mathrm{CHCO}), 2.40\left(\mathrm{~s}, 3 \mathrm{H}, \mathrm{CH}_{3}\right)$.

General procedure of bromination of $\alpha, \beta$-unsatured aromatic derivatives 30 and 31 . A solution of the corresponding $\alpha$-methyl ketones $\mathbf{3 0}$ and $\mathbf{3 1}$ in acetonitrile $(80 \mathrm{~mL}$ ) was reacted with $N$-bromosuccinimide in the presence of $p$-toluensulfonic acid at room temperature for $5 \mathrm{~h}$. Then, the reaction mixture was quenched with water $(20 \mathrm{~mL})$ and the 
solvent was removed at reduced pressure. The aqueous residue was extracted with ethyl acetate $(3 \times 20 \mathrm{~mL})$ and the combined organic layers were dried $\left(\mathrm{Na}_{2} \mathrm{SO}_{4}\right)$, filtered and evaporated to dryness. The final residue was purified by flash column chromatography using silica gel (hexane/ethyl acetate, $8: 2$ ) to give the appropriate $\alpha$-bromo methyl ketones compounds $\mathbf{3 4}$ and $\mathbf{3 5 .}$

(E)-1-bromo-4-(naphthalen-2-yl)but-3-en-2-one (34). ${ }^{4}$ Yield $46 \%{ }^{1} \mathrm{H}$ NMR $\left(\mathrm{CDCl}_{3}, 400\right.$ MHz) $\delta(\mathrm{ppm}): 8.00$ (d, $J=1.8 \mathrm{~Hz}, 1 \mathrm{H}, \mathrm{Ar}), 7.94-7.83$ (m, 4H, Ar, $\mathrm{C} \underline{H}=\mathrm{CHCO}), 7.71$ (dd, $J=8.5,1.8 \mathrm{~Hz}, 1 \mathrm{H}, \mathrm{Ar}), 7.66-7.49(\mathrm{~m}, 2 \mathrm{H}, \mathrm{Ar}), 7.07(\mathrm{~d}, J=15.9 \mathrm{~Hz}, 1 \mathrm{H}, \mathrm{CH}=\mathrm{C} \underline{\mathrm{HCO}})$, $4.12\left(\mathrm{~s}, 2 \mathrm{H}, \mathrm{CH}_{2} \mathrm{Br}\right) ;{ }^{13} \mathrm{C} \mathrm{NMR}\left(\mathrm{CDCl}_{3}, 75 \mathrm{MHz}\right) \delta(\mathrm{ppm}): 191.4(\mathrm{CO}), 145.6(\mathrm{Ar} \underline{\mathrm{CH}}=\mathrm{CH})$, $134.8\left(\mathrm{C}_{\mathrm{Ar}}\right), 133.4\left(\mathrm{C}_{\mathrm{Ar}}\right), 131.6\left(\mathrm{C}_{\mathrm{Ar}}\right), 131.3\left(\mathrm{CH}_{\mathrm{Ar}}\right), 129.0\left(\mathrm{CH}_{\mathrm{Ar}}\right), 128.9\left(\mathrm{CH}_{\mathrm{Ar}}\right), 128.0$ $\left(\mathrm{CH}_{\mathrm{Ar}}\right), 127.8(\mathrm{ArCH}=\underline{\mathrm{CH}}), 127.0\left(\mathrm{CH}_{\mathrm{Ar}}\right), 123.6\left(\mathrm{CH}_{\mathrm{Ar}}\right), 122.4\left(\mathrm{CH}_{\mathrm{Ar}}\right), 33.3\left(\mathrm{CH}_{2} \mathrm{Br}\right) ; \mathrm{MS}$ (ESI, positive) $m / z: 297[\mathrm{M}+\mathrm{Na}]^{+}, 275.0[\mathrm{M}+\mathrm{H}]^{+}$, both with a $\mathrm{Br}$ isotopic pattern

(E)-4-([1,1'-biphenyl]-4-yl)-1-bromobut-3-en-2-one (35). Yield 42\% ${ }^{1} \mathrm{H}$ NMR $\left(\mathrm{CDCl}_{3}\right.$, $400 \mathrm{MHz}) \delta(\mathrm{ppm}): 7.74(\mathrm{~d}, J=16.0 \mathrm{~Hz}, 1 \mathrm{H}, \mathrm{CH}=\mathrm{CHCO}), 7.67-7.55$ (m, 6H, Ar), 7.52 7.43 (m, 2H, Ar), 7.41 - 7.32 (m, 1H, Ar), 6.99 (d, J=16.0 Hz, 1H, CH=CㅍCO), 4.10 (s, $\left.2 \mathrm{H}, \mathrm{CH}_{2} \mathrm{Br}\right) ;{ }^{13} \mathrm{C} \mathrm{NMR}\left(\mathrm{CDCl}_{3}, 75 \mathrm{MHz}\right) \delta(\mathrm{ppm}): 191.1(\mathrm{CO}), 145.1(\mathrm{ArCH}=\mathrm{CH}), 144.0$ $\left(\mathrm{C}_{\mathrm{Ar}}\right), 140.1\left(\mathrm{C}_{\mathrm{Ar}}\right), 133.0\left(\mathrm{C}_{\mathrm{Ar}}\right), 129.3\left(\mathrm{CH}_{\mathrm{Ar}}\right), 129.1\left(\mathrm{CH}_{\mathrm{Ar}}\right), 128.2\left(\mathrm{CH}_{\mathrm{Ar}}\right), 127.8$ $(\mathrm{ArCH}=\underline{\mathrm{CH}}), 127.2\left(\mathrm{CH}_{\mathrm{Ar}}\right), 122.1\left(\mathrm{CH}_{\mathrm{Ar}}\right), 33.3\left(\mathrm{CH}_{2} \mathrm{Br}\right)$

\section{General procedure for the Hantzsch thiazole synthesis (36a-f)}

To a pressure flask was added the intermediate thioamide 27 ( 1 equiv) and the appropriated commercially available $\alpha$-haloketones or synthetic analogues 32-35 (1.1 equiv) in isopropanol $(25 \mathrm{~mL})$. The pressure flask was stirred at $70{ }^{\circ} \mathrm{C}$ for $4 \mathrm{~h}$ and the solvent was eliminated to dryness. The residue was purified by flash column chromatography using silica gel (DCM/MeOH, $9: 1)$ to give the thiazoles 36a-f as a colorless oil. 
tert-Butyl (2-((tert-butoxycarbonyl)aminoethyl)((1-(4-(4-isobutylthiazol-2-yl)-3-(2-(2oxoimidazolidin-1-yl)ethoxy)phenyl)-1H-imidazol-2-yl)methyl)carbamate (36a). Yield: 88 \%. ${ }^{1} \mathrm{H}$ NMR $\left(\mathrm{CD}_{3} \mathrm{OD}, 400 \mathrm{MHz}\right) \delta(\mathrm{ppm}): 8.41$ (d, $\left.J=7.1 \mathrm{~Hz}, 1 \mathrm{H}, \mathrm{Ar}\right), 7.42-7.35$ (m, 1H, Ar), $7.33(\mathrm{~s}, 1 \mathrm{H}, \mathrm{Ar}), 7.20(\mathrm{~s}, 1 \mathrm{H}, \mathrm{Ar}), 7.12-7.05(\mathrm{~m}, 2 \mathrm{H}, \mathrm{Ar}), 4.76-4.52(\mathrm{~m}, 2 \mathrm{H}$, $\left.\operatorname{ImC} \underline{H}_{2} \mathrm{~N}\right), 4.48-4.36\left(\mathrm{~m}, 2 \mathrm{H}, \mathrm{OCH}_{2} \mathrm{CH}_{2}\right), 3.74\left(\mathrm{t}, J=5.4 \mathrm{~Hz}, 2 \mathrm{H}, \mathrm{OCH}_{2} \underline{\mathrm{CH}}_{2}\right), 3.62(\mathrm{t}, J=$ $\left.8.0 \mathrm{~Hz}, 2 \mathrm{H}, \mathrm{CH}_{2} \mathrm{CH}_{2} \mathrm{NHCON}\right), 3.37$ (dd, $J=9.0,7.0 \mathrm{~Hz}, 2 \mathrm{H}, \mathrm{CH}_{2} \underline{\mathrm{CH}}_{2} \mathrm{NHCON}, 3.28-$ $3.05\left(\mathrm{~m}, 4 \mathrm{H}, \underline{\mathrm{CH}}_{2} \underline{\mathrm{C}}_{2}{ }_{2} \mathrm{NHBoc}\right), 2.70\left(\mathrm{~d}, J=7.1 \mathrm{~Hz}, 2 \mathrm{H}, \underline{\mathrm{CH}}_{2} \mathrm{CH}\left(\mathrm{CH}_{3}\right)_{2}\right), 2.18-2.05$ (m, $\left.1 \mathrm{H}, \mathrm{CH}_{2} \mathrm{C} \underline{\mathrm{H}}\left(\mathrm{CH}_{3}\right)_{2}\right), 1.38\left(\mathrm{~s}, 9 \mathrm{H}, \mathrm{CH}_{3}\right), 1.27\left(\mathrm{~s}, 9 \mathrm{H}, \mathrm{CH}_{3}\right), 0.97(\mathrm{~d}, J=6.6 \mathrm{~Hz}, 6 \mathrm{H}$, $\left.\mathrm{CH}_{2} \mathrm{CH}\left(\mathrm{CH}_{3}\right)_{2}\right) ;{ }^{13} \mathrm{C} \mathrm{NMR}\left(\mathrm{CD}_{3} \mathrm{OD}, 75 \mathrm{MHz}\right) \delta(\mathrm{ppm}): 165.0(\mathrm{NHCON}), 162.0\left(\mathrm{C}_{\mathrm{Ar}}\right)$, $158.2\left(\mathrm{OC}_{\mathrm{Ar}}\right), 157.1(\mathrm{NHCOO}), 156.5(\mathrm{NHCOO}), 146.3\left(\mathrm{C}_{\mathrm{Ar}}\right), 145.9\left(\mathrm{C}_{\mathrm{Ar}}\right), 139.8\left(\mathrm{CH}_{\mathrm{Ar}}\right)$, $130.3\left(\mathrm{C}_{\mathrm{Ar}}\right), 128.2\left(\mathrm{CH}_{\mathrm{Ar}}\right), 123.6\left(\mathrm{CH}_{\mathrm{Im}}\right), 123.6\left(\mathrm{CH}_{\mathrm{Im}}\right), 119.1\left(\mathrm{CH}_{\mathrm{Ar}}\right), 117.2\left(\mathrm{CH}_{\mathrm{Ar}}\right), 111.7$ $\left(\mathrm{C}_{\mathrm{Ar}}\right), 81.5\left(\mathrm{OC}\left(\mathrm{CH}_{3}\right)_{3}\right), 79.9\left(\mathrm{O} \underline{\mathrm{C}}\left(\mathrm{CH}_{3}\right)_{3}\right), 68.4\left(\mathrm{OCH}_{2}\right), 47.3\left(\underline{\mathrm{CH}_{2}} \mathrm{CH}_{2} \mathrm{NHCON}\right), 46.8$ $\left(\mathrm{OCH}_{2} \underline{\mathrm{CH}_{2}}\right), 43.8\left(\underline{\mathrm{CH}}_{2} \mathrm{CH}_{2} \mathrm{NHBoc}\right), 41.4\left(\mathrm{CH}_{2} \underline{\mathrm{CH}}_{2} \mathrm{NHBoc}\right), 39.3\left(\mathrm{CH}_{2} \underline{\mathrm{CH}}_{2} \mathrm{NHCON}\right), 29.9$ (ThiazCH $\left.\mathrm{CH}_{2}\right), 28.8\left(\mathrm{CH}_{3}\right), 28.5\left(\mathrm{CH}_{3}\right), 22.8\left(\left(\mathrm{CH}_{3}\right)_{2}\right)$; HRMS (ESI positive, $\left.m / z\right)$ : calculated for $\mathrm{C}_{34} \mathrm{H}_{49} \mathrm{~N}_{7} \mathrm{O}_{6} \mathrm{~S}$ 683.3465; found 683.3468 (0.37 ppm)

tert-Butyl (2-((tert-butoxycarbonyl)aminoethyl)((1-(4-(4-neopentylthiazol-2-yl)-3-(2-(2oxoimidazolidin-1-yl)ethoxy)phenyl)-1H-imidazol-2-yl)methyl)carbamate (36b). Yield: 75 \%. ${ }^{1} \mathrm{H}$ NMR $\left(\mathrm{CD}_{3} \mathrm{OD}, 400 \mathrm{MHz}\right) \delta(\mathrm{ppm}): 8.42(\mathrm{~d}, J=7.1 \mathrm{~Hz}, 1 \mathrm{H}, \mathrm{Ar}), 7.42-7.35(\mathrm{~m}$, 1H, Ar), $7.33(\mathrm{~s}, 1 \mathrm{H}, \mathrm{Ar}), 7.20(\mathrm{~s}, 1 \mathrm{H}, \mathrm{Ar}), 7.12-7.05(\mathrm{~m}, \mathrm{Ar}), 4.76-4.51(\mathrm{~m}, 2 \mathrm{H}$, $\left.\mathrm{ImC} \underline{\mathrm{H}}_{2} \mathrm{~N}\right), 4.48-4.37\left(\mathrm{~m}, 2 \mathrm{H}, \mathrm{OCH}_{2} \mathrm{CH}_{2}\right), 3.75\left(\mathrm{t}, J=5.4 \mathrm{~Hz}, 2 \mathrm{H}, \mathrm{OCH}_{2} \underline{\mathrm{CH}}_{2}\right), 3.63(\mathrm{t}, J=$ $\left.8.0 \mathrm{~Hz}, 2 \mathrm{H}, \mathrm{CH}_{2} \mathrm{CH}_{2} \mathrm{NHCON}\right), 3.38$ (dd, $\left.J=8.9,6.9 \mathrm{~Hz}, 2 \mathrm{H}, \mathrm{CH}_{2} \underline{\mathrm{CH}}_{2} \mathrm{NHCON}\right), 3.28-$ $3.03\left(\mathrm{~m}, 4 \mathrm{H}, \underline{\mathrm{C}}_{2} \mathrm{C}_{2}{ }_{2} \mathrm{NHBoc}\right), 2.75\left(\mathrm{~s}, 2 \mathrm{H}, \mathrm{CH}_{2} \underline{\mathrm{C}}\left(\mathrm{CH}_{3}\right)_{3}\right), 1.39$ (s, 9H, $\left.\mathrm{CH}_{3}\right), 1.26(\mathrm{~s}, 9 \mathrm{H}$, $\left.\mathrm{CH}_{3}\right), 0.99$ (s, 9H, $\left.\mathrm{CH}_{2} \mathrm{C}\left(\mathrm{CH}_{3}\right)_{3}\right) ;{ }^{13} \mathrm{C} \mathrm{NMR}\left(\mathrm{CD}_{3} \mathrm{OD}, 75 \mathrm{MHz}\right) \delta(\mathrm{ppm}): 166.0(\mathrm{NHCON})$, $165.1\left(\mathrm{C}_{\mathrm{Ar}}\right), 158.2\left(\mathrm{C}_{\mathrm{Ar}}\right), 157.1(\mathrm{NHCOO}), 156.5(\mathrm{NHCOO}), 155.8\left(\mathrm{C}_{\mathrm{Ar}}\right), 145.9\left(\mathrm{C}_{\mathrm{Ar}}\right)$, $139.8\left(\mathrm{CH}_{\mathrm{Ar}}\right), 130.3\left(\mathrm{C}_{\mathrm{Ar}}\right), 128.3\left(\mathrm{CH}_{\mathrm{Ar}}\right), 124.1\left(\mathrm{CH}_{\mathrm{Im}}\right), 123.6\left(\mathrm{CH}_{\mathrm{Im}}\right), 119.2\left(\mathrm{CH}_{\mathrm{Ar}}\right), 118.4$ 
$\left(\mathrm{CH}_{\mathrm{Ar}}\right), \quad 111.7 \quad\left(\mathrm{C}_{\mathrm{Ar}}\right), \quad 81.5 \quad\left(\mathrm{OC}\left(\mathrm{CH}_{3}\right)_{3}\right), \quad 79.9 \quad\left(\mathrm{OC}\left(\mathrm{CH}_{3}\right)_{3}\right), \quad 68.3 \quad\left(\mathrm{OCH}_{2}\right), \quad 47.3$ $\left(\underline{\mathrm{CH}}_{2} \mathrm{CH}_{2} \mathrm{NHCON}\right), 46.8\left(\mathrm{OCH}_{2} \underline{\mathrm{CH}_{2}}\right), 45.7\left(\underline{\mathrm{CH}}_{2} \mathrm{CH}_{2} \mathrm{NHBoc}\right), 43.9\left(\mathrm{CH}_{2} \mathrm{CH}_{2} \mathrm{NHBoc}\right), 39.3$ $\left(\mathrm{CH}_{2} \mathrm{CH}_{2} \mathrm{NHCON}\right), 32.4$ (ThiazCH $\left.2 \mathrm{C}\right), 30.0\left(\mathrm{ThiazCH}_{2}\right), 28.8\left(\mathrm{CH}_{3}\right), 28.6\left(\mathrm{CH}_{3}\right) ; \mathrm{HRMS}$ (ESI positive, $m / z$ ): calculated for $\mathrm{C}_{35} \mathrm{H}_{51} \mathrm{~N}_{7} \mathrm{O}_{6} \mathrm{~S}$ 697.3621; found 697.3644 (3.19 ppm)

tert-Butyl ((1-(4-(4-benzylthiazol-2-yl)-3-(2-(2-oxoimidazolidin-1-yl)ethoxy)phenyl)-1Himidazol-2-yl)methyl)(2-((tert-butoxycarbonyl)amino)ethyl)carbamate (36c). Yield: $95 \%$. ${ }^{1} \mathrm{H}$ NMR $\left(\mathrm{CD}_{3} \mathrm{OD}, 400 \mathrm{MHz}\right) \delta(\mathrm{ppm}): 8.39(\mathrm{~d}, J=7.1 \mathrm{~Hz}, 1 \mathrm{H}, \mathrm{Ar}), 7.36-7.26(\mathrm{~m}, 5 \mathrm{H}$, Ar), $7.24-7.16(\mathrm{~m}, 1 \mathrm{H}, \mathrm{Ar}), 7.33(\mathrm{~s}, 1 \mathrm{H}, \mathrm{Ar}), 7.20(\mathrm{~s}, 1 \mathrm{H}, \mathrm{Ar}), 7.12-7.05$ (m, 2H, Ar), $4.72-4.53\left(\mathrm{~m}, 2 \mathrm{H}, \mathrm{ImCH}_{2} \mathrm{~N}\right), 4.45-4.38\left(\mathrm{~m}, 2 \mathrm{H}, \mathrm{OCH}_{2} \mathrm{CH}_{2}\right), 4.17\left(\mathrm{~s}, 2 \mathrm{H}, \mathrm{CH}_{2} \mathrm{Ph}\right), 3.72$ (t, $\left.J=5.4 \mathrm{~Hz}, 2 \mathrm{H}, \mathrm{OCH}_{2} \mathrm{CH}_{2}\right), 3.58\left(\mathrm{t}, J=8.1 \mathrm{~Hz}, 2 \mathrm{H}, \mathrm{CH}_{2} \mathrm{CH}_{2} \mathrm{NHCON}, 3.35\right.$ (dd, $J=$ 8.8, $\left.6.8 \mathrm{~Hz}, 2 \mathrm{H}, \mathrm{CH}_{2} \underline{\mathrm{C}}_{2} \mathrm{NHCON}\right), 3.29-3.06$ (m, 4H, $\underline{\mathrm{CH}}_{2} \mathrm{CH}_{2} \mathrm{NHBoc}, 1.39$ (s, 9H, $\left.\mathrm{CH}_{3}\right), 1.25\left(\mathrm{~s}, 9 \mathrm{H}, \mathrm{CH}_{3}\right) ;{ }^{13} \mathrm{C} \mathrm{NMR}\left(\mathrm{CD}_{3} \mathrm{OD}, 75 \mathrm{MHz}\right) \delta(\mathrm{ppm}): 165.0(\mathrm{NHCON}), 162.5$ $\left(\mathrm{C}_{\mathrm{Ar}}\right), 158.2\left(\mathrm{C}_{\mathrm{Ar}}\right), 157.1(\mathrm{NHCOO}), 156.9(\mathrm{NHCOO}), 156.5\left(\mathrm{C}_{\mathrm{Ar}}\right), 145.8\left(\mathrm{C}_{\mathrm{Ar}}\right), 140.9$ $\left(\mathrm{C}_{\mathrm{Ar}}\right), 140.0\left(\mathrm{CH}_{\mathrm{Ar}}\right), 130.3\left(\mathrm{C}_{\mathrm{Ar}}\right), 129.5\left(\mathrm{CH}_{\mathrm{Ar}}\right), 128.3\left(\mathrm{CH}_{\mathrm{Ar}}\right), 127.3\left(\mathrm{CH}_{\mathrm{Ar}}\right), 123.6\left(\mathrm{CH}_{\mathrm{Im}}\right)$, $123.6\left(\mathrm{CH}_{\mathrm{Im}}\right), \quad 119.1\left(\mathrm{CH}_{\mathrm{Ar}}\right), \quad 117.6\left(\mathrm{CH}_{\mathrm{Ar}}\right), \quad 111.7\left(\mathrm{C}_{\mathrm{Ar}}\right), 81.5 \quad\left(\mathrm{OC}\left(\mathrm{CH}_{3}\right)_{3}\right), \quad 79.9$ $\left(\mathrm{OC}\left(\mathrm{CH}_{3}\right)_{3}\right), \quad 68.3 \quad\left(\mathrm{OCH}_{2}\right), \quad 47.2 \quad\left(\underline{\mathrm{CH}}_{2} \mathrm{CH}_{2} \mathrm{NHCON}\right), \quad 46.8 \quad\left(\mathrm{OCH}_{2} \underline{\mathrm{CH}}_{2}\right), \quad 43.8$ $\left(\mathrm{CH}_{2} \mathrm{CH}_{2} \mathrm{NHBoc}\right), 39.2\left(\mathrm{CH}_{2} \mathrm{CH}_{2} \mathrm{NHCON}\right), 38.4\left(\mathrm{ThiazCH}_{2}\right), 28.8\left(\mathrm{CH}_{3}\right), 28.5\left(\mathrm{CH}_{3}\right)$; HRMS (ESI positive, $m / z$ ): calculated for $\mathrm{C}_{33} \mathrm{H}_{47} \mathrm{~N}_{7} \mathrm{O}_{6} \mathrm{~S}$ 717.3308; found 717.3343 (4.85 ppm)

tert-Butyl (2-((tert-butoxycarbonyl)amino)ethyl)((1-(3-(2-(2-oxoimidazolidin-1-yl)ethoxy)(4-(4-phenetylthiazol-2-yl)phenyl)-1H-imidazol-2-yl)methyl)carbamate (36d). Yield: 94 \%. ${ }^{1} \mathrm{H}$ NMR $\left(\mathrm{CD}_{3} \mathrm{OD}, 400 \mathrm{MHz}\right) \delta(\mathrm{ppm}): 8.44(\mathrm{~d}, J=6.8 \mathrm{~Hz}, 1 \mathrm{H}, \mathrm{Ar}), 7.43-7.37(\mathrm{~m}, 1 \mathrm{H}$, Ar), $7.33(\mathrm{~s}, 1 \mathrm{H}, \mathrm{Ar}), 7.26-7.21(\mathrm{~m}, 2 \mathrm{H}, \mathrm{Ar}), 7.20-7.10(\mathrm{~m}, 5 \mathrm{H}, \mathrm{Ar}), 7.08(\mathrm{~s}, 1 \mathrm{H}, \mathrm{Ar})$, $4.75-4.55\left(\mathrm{~m}, 2 \mathrm{H}, \mathrm{ImCH}_{2} \mathrm{~N}\right), 4.47-4.41\left(\mathrm{~m}, 2 \mathrm{H}, \mathrm{OCH}_{2} \mathrm{CH}_{2}\right), 3.74(\mathrm{t}, J=4.9 \mathrm{~Hz}, 2 \mathrm{H}$, $\left.\mathrm{OCH}_{2} \mathrm{CH}_{2}\right), 3.61\left(\mathrm{t}, J=8.1 \mathrm{~Hz}, 2 \mathrm{H}, \mathrm{CH}_{2} \mathrm{CH}_{2} \mathrm{NHCON}\right), 3.38(\mathrm{dd}, J=9.1,7.0 \mathrm{~Hz}, 2 \mathrm{H}$, 
$\left.\mathrm{CH}_{2} \underline{\mathrm{CH}}_{2} \mathrm{NHCON}\right), 3.16$ - 3.06 (m, 8H, $\left.\underline{\mathrm{CH}}_{2} \underline{\mathrm{CH}}_{2} \mathrm{NHBoc}, \underline{\mathrm{CH}}_{2} \underline{\mathrm{CH}}_{2} \mathrm{Ph}\right), 1.39$ (s, 9H, $\left.\mathrm{CH}_{3}\right)$, $1.26\left(\mathrm{~s}, 9 \mathrm{H}, \mathrm{CH}_{3}\right) ;{ }^{13} \mathrm{C} \mathrm{NMR}\left(\mathrm{CD}_{3} \mathrm{OD}, 75 \mathrm{MHz}\right) \delta(\mathrm{ppm}): 166.1(\mathrm{NHCON}), 165.0\left(\mathrm{C}_{\mathrm{Ar}}\right)$, $158.3\left(\mathrm{C}_{\mathrm{Ar}}\right), 157.1(\mathrm{NHCOO}), 156.9(\mathrm{NHCOO}), 156.5\left(\mathrm{C}_{\mathrm{Ar}}\right), 145.9\left(\mathrm{C}_{\mathrm{Ar}}\right), 142.8\left(\mathrm{C}_{\mathrm{Ar}}\right)$, $140.0\left(\mathrm{CH}_{\mathrm{Ar}}\right), 130.3\left(\mathrm{C}_{\mathrm{Ar}}\right), 129.5\left(\mathrm{CH}_{\mathrm{Ar}}\right), 129.3\left(\mathrm{CH}_{\mathrm{Ar}}\right), 128.3\left(\mathrm{CH}_{\mathrm{Ar}}\right), 127.0\left(\mathrm{CH}_{\mathrm{Ar}}\right), 123.6$ $\left(\mathrm{CH}_{\mathrm{Im}}\right), 123.6\left(\mathrm{CH}_{\mathrm{Im}}\right), 119.1\left(\mathrm{CH}_{\mathrm{Ar}}\right), 117.0\left(\mathrm{CH}_{\mathrm{Ar}}\right), 111.7\left(\mathrm{C}_{\mathrm{Ar}}\right), 81.5\left(\mathrm{OC}\left(\mathrm{CH}_{3}\right)_{3}\right), 80.0$ $\left(\mathrm{OC}\left(\mathrm{CH}_{3}\right)_{3}\right), \quad 68.3 \quad\left(\mathrm{OCH}_{2}\right), \quad 47.2 \quad\left(\underline{\mathrm{CH}}_{2} \mathrm{CH}_{2} \mathrm{NHCON}\right), \quad 46.8 \quad\left(\mathrm{OCH}_{2} \underline{\mathrm{CH}_{2}}\right), \quad 43.8$ $\left(\mathrm{CH}_{2} \mathrm{CH}_{2} \mathrm{NHBoc}\right), 39.3\left(\mathrm{CH}_{2} \mathrm{CH}_{2} \mathrm{NHCON}\right), 36.7\left(\mathrm{ThiazCH}_{2} \mathrm{CH}_{2}\right), 34.3\left(\mathrm{ThiazCH}_{2} \mathrm{CH}_{2}\right)$, $28.8\left(\mathrm{CH}_{3}\right), 28.6\left(\mathrm{CH}_{3}\right)$; HRMS (ESI positive, $\left.m / z\right)$ : calculated for $\mathrm{C}_{38} \mathrm{H}_{49} \mathrm{~N}_{7} \mathrm{O}_{6} \mathrm{~S}$ 731.3465; found $731.3483(2.42 \mathrm{ppm})$

tert-Butyl (E)-(2-((tert-butoxycarbonyl)amino)ethyl)((1-(4-(4-(2-(naphthalen-2yl)vinyl)thiazol-2-yl)-3-(2-(2-oxoimidazolidin-1-yl)ethoxy)phenyl)-1H-imidazol-2-

yl)methyl)carbamate (36e). Yield: $69 \% .{ }^{1} \mathrm{H}$ NMR $\left(\mathrm{CD}_{3} \mathrm{OD}, 400 \mathrm{MHz}\right) \delta(\mathrm{ppm}): 8.60(\mathrm{~d}, J$ $=8.3 \mathrm{~Hz}, 1 \mathrm{H}, \mathrm{Ar}), 8.05(\mathrm{~s}, 1 \mathrm{H}, \mathrm{Ar}), 7.93(\mathrm{~s}, 1 \mathrm{H}, \mathrm{Ar}), 7.90-7.77(\mathrm{~m}, 4 \mathrm{H}, \mathrm{Ar}), 7.73(\mathrm{~d}, J=$ $16.0 \mathrm{~Hz}, 1 \mathrm{H}, \mathrm{CH}=\mathrm{CH}), 7.58$ (s, 1H, Ar), $7.49-7.32$ (m, 5H, Ar), 7.15 (dd, $J=8.3,1.9 \mathrm{~Hz}$, $1 \mathrm{H}, \mathrm{Ar}) 7.09$ (s, 1H, Ar), $4.78-4.52\left(\mathrm{~m}, 2 \mathrm{H}, \mathrm{ImC}_{2} \underline{\mathrm{N}}\right), 4.49-4.42\left(\mathrm{~m}, 2 \mathrm{H}, \mathrm{OC}_{\underline{\mathrm{H}}} \mathrm{CH}_{2}\right)$, $3.76\left(\mathrm{t}, J=5.5 \mathrm{~Hz}, 2 \mathrm{H}, \mathrm{OCH}_{2} \mathrm{CH}_{2}\right), 3.63\left(\mathrm{t}, J=8.2 \mathrm{~Hz}, 2 \mathrm{H}, \mathrm{CH}_{2} \mathrm{CH}_{2} \mathrm{NHCON}\right), 3.37$ (dd, $J$ $\left.=9.0,6.9 \mathrm{~Hz}, 2 \mathrm{H}, \mathrm{CH}_{2} \underline{\mathrm{C}}_{2} \mathrm{NHCON}\right), 3.25-3.04$ (m, 4H, $\left.\underline{\mathrm{CH}}_{2} \underline{\mathrm{CH}}_{2} \mathrm{NHBoc}\right), 1.39$ (s, 9H, $\left.\mathrm{CH}_{3}\right), 1.28\left(\mathrm{~s}, 9 \mathrm{H}, \mathrm{CH}_{3}\right) ;{ }^{13} \mathrm{C} \mathrm{NMR}\left(\mathrm{CD}_{3} \mathrm{OD}, 75 \mathrm{MHz}\right) \delta(\mathrm{ppm}): 166.5$ (NHCOO), 165.3 $\left(\mathrm{C}_{\mathrm{Ar}}\right), 162.8\left(\mathrm{C}_{\mathrm{Ar}}\right), 158.7\left(\mathrm{C}_{\mathrm{Ar}}\right), 157.7(\mathrm{NHCOO}), 157.0(\mathrm{NHCOO}), 155.1\left(\mathrm{C}_{\mathrm{Ar}}\right), 140.6$ $\left(\mathrm{C}_{\mathrm{Ar}}\right), 136.5\left(\mathrm{C}_{\mathrm{Ar}}\right), 135.7\left(\mathrm{CH}_{\mathrm{Ar}}\right), 135.1\left(\mathrm{C}_{\mathrm{Ar}}\right), 132.7\left(\mathrm{C}_{\mathrm{Ar}}\right), 131.2\left(\mathrm{CH}_{\mathrm{Ar}}\right), 129.8\left(\mathrm{CH}_{\mathrm{Ar}}\right)$, $129.5\left(\mathrm{CH}_{\mathrm{Ar}}\right), 129.1\left(\mathrm{CH}_{\mathrm{Ar}}\right), 128.7\left(\mathrm{CH}_{\mathrm{Ar}}\right), 128.4\left(\mathrm{CH}_{\mathrm{Ar}}\right), 127.8\left(\mathrm{CH}_{\mathrm{Ar}}\right), 127.5\left(\mathrm{C}_{\mathrm{Ar}}\right), 124.8$ $\left(\mathrm{CH}_{\mathrm{Ar}}\right) .123 .9\left(\mathrm{CH}_{\mathrm{Ar}}\right), 123.4\left(\mathrm{CH}_{\mathrm{Im}}\right), 123.4\left(\mathrm{CH}_{\mathrm{Im}}\right), 119.1\left(\mathrm{CH}_{\mathrm{Ar}}\right), 112.1\left(\mathrm{C}_{\mathrm{Ar}}\right), 81.9$ $\left(\mathrm{OC}\left(\mathrm{CH}_{3}\right)_{3}\right), 80.4\left(\mathrm{OC}\left(\mathrm{CH}_{3}\right)_{3}\right), 68.7\left(\mathrm{OCH}_{2}\right), 47.2\left(\mathrm{OCH}_{2} \underline{\mathrm{CH}_{2}}\right), 44.3\left(\mathrm{CH}_{2} \mathrm{CH}_{2} \mathrm{NHBoc}\right)$,

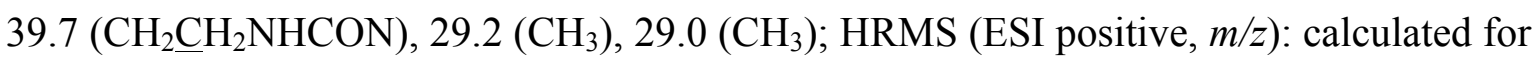
$\mathrm{C}_{42} \mathrm{H}_{49} \mathrm{~N}_{7} \mathrm{O}_{6} \mathrm{~S} \quad 779.3465$; found 779.3474 (1.20 ppm) 
tert-Butyl

(E)-((1-(4-(4-(2-([1,1'-biphenyl]-4-yl) vinyl)thiazol-2-yl)-3-(2-(2-

oxoimidazolidin-1-yl)ethoxy)phenyl)-1H-imidazol-2-yl)methyl)(2-((tert-

butoxycarbonyl)amino)ethyl)carbamate (36f). Yield: $79 \% .{ }^{1} \mathrm{H} \mathrm{NMR}\left(\mathrm{CDCl}_{3}, 400 \mathrm{MHz}\right) \delta$ (ppm): $8.62(\mathrm{~d}, J=8.3 \mathrm{~Hz}, 1 \mathrm{H}, \mathrm{Ar}), 7.84-7.54(\mathrm{~m}, 7 \mathrm{H}, \mathrm{Ar}), 7.48-7.52$ (m, 2H, Ar), 7.41 - $7.24(\mathrm{~m}, 2 \mathrm{H}, \mathrm{Ar}), 7.21(\mathrm{~d}, J=16.0 \mathrm{~Hz}, 1 \mathrm{H}, \mathrm{CH}=\mathrm{CH}), 7.15-6.90(\mathrm{~m}, 3 \mathrm{H}, \mathrm{Ar}), 4.66-4.38$ (m, 4H, ImC $\left.\underline{H}_{2} \mathrm{NH}, \mathrm{OC}_{2} \mathrm{CH}_{2}\right), 3.80\left(\mathrm{t}, J=5.5 \mathrm{~Hz}, 2 \mathrm{H}, \mathrm{OCH}_{2} \underline{\mathrm{CH}}_{2}\right), 3.61(\mathrm{t}, J=7.8 \mathrm{~Hz}$, $\left.2 \mathrm{H}, \mathrm{CH}_{2} \mathrm{CH}_{2} \mathrm{NHCON}\right), 3.46-3.33$ (m, 4H, $\mathrm{CH}_{2} \mathrm{CH}_{2} \mathrm{NHCON}, \mathrm{CH}_{2} \mathrm{CH}_{2} \mathrm{NHBoc}$, 3.27 $3.19\left(\mathrm{~m}, 2 \mathrm{H}, \mathrm{C}_{2} \mathrm{CH}_{2} \mathrm{NHBoc}\right), 1.43\left(\mathrm{~s}, 9 \mathrm{H}, \mathrm{CH}_{3}\right), 1.37\left(\mathrm{~s}, 9 \mathrm{H}, \mathrm{CH}_{3}\right) ;{ }^{13} \mathrm{C} \mathrm{NMR}\left(\mathrm{CDCl}_{3}, 75\right.$ MHz) $\delta(\mathrm{ppm}): 162.7(\mathrm{NHCOO}), 161.0\left(\mathrm{C}_{\mathrm{Ar}}\right), 156.3\left(\mathrm{C}_{\mathrm{Ar}}\right), 155.9(\mathrm{NHCOO}), 155.4$ (NHCOO), $153.4\left(\mathrm{C}_{\mathrm{Ar}}\right), 144.9\left(\mathrm{C}_{\mathrm{Ar}}\right), 140.7\left(\mathrm{C}_{\mathrm{Ar}}\right), 140.7\left(\mathrm{C}_{\mathrm{Ar}}\right), 139.0\left(\mathrm{CH}_{\mathrm{Ar}}\right), 136.2\left(\mathrm{C}_{\mathrm{Ar}}\right)$, $131.0\left(\mathrm{CH}_{\mathrm{Ar}}\right), 130.0\left(\mathrm{CH}_{\mathrm{Ar}}\right), 128.9\left(\mathrm{CH}_{\mathrm{Ar}}\right), 128.3\left(\mathrm{CH}_{\mathrm{Ar}}\right), 127.5\left(\mathrm{CH}_{\mathrm{Ar}}\right), 127.2\left(\mathrm{CH}_{\mathrm{Ar}}\right)$, $127.0\left(\mathrm{CH}_{\mathrm{Ar}}\right), 122.6\left(\mathrm{CH}_{\mathrm{Im}}\right), 121.5\left(\mathrm{CH}_{\mathrm{Im}}\right), 118.3\left(\mathrm{CH}_{\mathrm{Ar}}\right), 117.2\left(\mathrm{CH}_{\mathrm{Ar}}\right), 110.2\left(\mathrm{C}_{\mathrm{Ar}}\right), 80.5$ $\left(\mathrm{OC}\left(\mathrm{CH}_{3}\right)_{3}\right), 78.9\left(\mathrm{OC}\left(\mathrm{CH}_{3}\right)_{3}\right), 67.9\left(\mathrm{OCH}_{2}\right), 48.4\left(\mathrm{CH}_{2} \mathrm{CH}_{2} \mathrm{NHCON}\right), 46.3\left(\mathrm{OCH}_{2} \mathrm{CH}_{2}\right)$, $43.2\left(\mathrm{CH}_{2} \mathrm{CH}_{2} \mathrm{NHBoc}\right), 39.4\left(\mathrm{CH}_{2} \mathrm{CH}_{2} \mathrm{NHCON}\right), 38.4\left(\mathrm{CH}_{2} \mathrm{CH}_{2} \mathrm{NHBoc}\right), 28.6\left(\mathrm{CH}_{3}\right), 28.3$ $\left(\mathrm{CH}_{3}\right)$; HRMS (ESI positive, $m / z$ ): calculated for $\mathrm{C}_{44} \mathrm{H}_{51} \mathrm{~N}_{7} \mathrm{O}_{6} \mathrm{~S}$ 805.3622; found 805.3628 (0.83 ppm).

\section{General procedure for hydrogenation of the double bond of $36 e, f$}

A solution of the unsaturated analogues 36e,f (1 equiv) in THF/MeOH, 1:1 (30 mL) containing $\mathrm{Pd} / \mathrm{C}(10 \%)(20 \% \mathrm{wt} / \mathrm{wt})$ was hydrogenated at room temperature for $2 \mathrm{~h}$ under atmospheric pressure using a balloon filled with hydrogen gas (3 cycles of vacuum + hydrogen). The $\mathrm{Pd} / \mathrm{C}$ was filtered through Whatman PTFE filter paper and the solvent was removed under reduced pressure. The residue was purified by CCTLC on the Chromatotron (DCM/MeOH, 9.5:0.5) to afford 37e,f as a colorless oil.

tert-Butyl

(2-((tert-butoxycarbonyl)amino)ethyl)((1-(4-(4-(2-(naphthalen-2-

yl)ethyl)thiazol-2-yl)-3-(2-(2-oxoimidazolidin-1-yl)ethoxy)phenyl)-1H-imidazol-2- 
yl)methyl) carbamate (37e). Yield: $70 \% .{ }^{1} \mathrm{H}$ NMR $\left(\mathrm{CD}_{3} \mathrm{OD}, 400 \mathrm{MHz}\right) \delta(\mathrm{ppm}): 8.43(\mathrm{~d}, J$ $=8.3 \mathrm{~Hz}, 1 \mathrm{H}), 7.87-7.69(\mathrm{~m}, 3 \mathrm{H}), 7.62(\mathrm{~d}, J=1.7 \mathrm{~Hz}, 1 \mathrm{H}), 7.46-6.93(\mathrm{~m}, 8 \mathrm{H}), 4.72-$ $4.52\left(\mathrm{~m}, 2 \mathrm{H}, \mathrm{ImCH}_{2} \mathrm{~N}\right), 4.49-4.42\left(\mathrm{~m}, 2 \mathrm{H}, \mathrm{OC}_{2} \mathrm{CH}_{2}\right), 3.71(\mathrm{t}, J=5.1 \mathrm{~Hz}, 2 \mathrm{H}$, $\left.\mathrm{OCH}_{2} \mathrm{CH}_{2}\right), 3.57$ (t, $\left.J=8.0 \mathrm{~Hz}, 2 \mathrm{H}, \mathrm{CH}_{2} \mathrm{CH}_{2} \mathrm{NHCON}\right), 3.34-3.28(\mathrm{~m}, 2 \mathrm{H}$, $\left.\mathrm{CH}_{2} \mathrm{CH}_{2} \mathrm{NHCON}\right), 3.25-3.22$ (m, 4H, $\left.\mathrm{CH}_{2} \mathrm{CH}_{2} \mathrm{NHBoc}\right), 3.19-3.06$ (m, $4 \mathrm{H}$, $\left.\mathrm{CH}_{2} \mathrm{CH}_{2} \mathrm{Naft}\right), 1.38$ (s, 9H, CH $), 1.26\left(\mathrm{~s}, 9 \mathrm{H}, \mathrm{CH}_{3}\right) ;{ }^{13} \mathrm{C} \mathrm{NMR}\left(\mathrm{CD}_{3} \mathrm{OD}, 75 \mathrm{MHz}\right) \delta(\mathrm{ppm})$ : $166.0(\mathrm{NHCON}), 165.0\left(\mathrm{C}_{\mathrm{Ar}}\right), 162.1\left(\mathrm{C}_{\mathrm{Ar}}\right), 158.2\left(\mathrm{C}_{\mathrm{Ar}}\right), 157.1(\mathrm{NHCOO}), 156.9(\mathrm{NHCOO})$, $156.5\left(\mathrm{C}_{\mathrm{Ar}}\right), 140.3\left(\mathrm{C}_{\mathrm{Ar}}\right), 139.9\left(\mathrm{C}_{\mathrm{Ar}}\right), 135.1\left(\mathrm{C}_{\mathrm{Ar}}\right), 133.6\left(\mathrm{C}_{\mathrm{Ar}}\right), 130.3\left(\mathrm{CH}_{\mathrm{Ar}}\right), 128.8\left(\mathrm{CH}_{\mathrm{Ar}}\right)$, 128.6 $\left(\mathrm{CH}_{\mathrm{Ar}}\right), 128.5\left(\mathrm{CH}_{\mathrm{Ar}}\right), 128.3\left(\mathrm{CH}_{\mathrm{Ar}}\right), 127.6\left(\mathrm{C}_{\mathrm{Ar}}\right), 126.9\left(\mathrm{CH}_{\mathrm{Ar}}\right), 126.2\left(\mathrm{CH}_{\mathrm{Ar}}\right), 123.5$ $\left(\mathrm{CH}_{\mathrm{Im}}\right), 123.5\left(\mathrm{CH}_{\mathrm{Im}}\right), 119.1\left(\mathrm{CH}_{\mathrm{Ar}}\right), 117.1\left(\mathrm{CH}_{\mathrm{Ar}}\right), 111.7\left(\mathrm{C}_{\mathrm{Ar}}\right), 81.5\left(\mathrm{OC}\left(\mathrm{CH}_{3}\right)_{3}\right), 79.9$ $\left(\mathrm{OC}\left(\mathrm{CH}_{3}\right)_{3}\right), \quad 68.4 \quad\left(\mathrm{OCH}_{2}\right), \quad 47.4 \quad\left(\mathrm{CH}_{2} \mathrm{CH}_{2} \mathrm{NHCON}\right), \quad 46.8 \quad\left(\mathrm{OCH}_{2} \mathrm{CH}_{2}\right), \quad 43.8$ $\left(\mathrm{CH}_{2} \mathrm{CH}_{2} \mathrm{NHBoc}\right), 39.2\left(\mathrm{CH}_{2} \mathrm{CH}_{2} \mathrm{NHCON}\right), 36.8\left(\mathrm{ThiazCH}_{2} \mathrm{CH}_{2}\right), 34.0\left(\mathrm{ThiazCH}_{2} \mathrm{CH}_{2}\right)$, $28.8\left(\mathrm{CH}_{3}\right), 28.6\left(\mathrm{CH}_{3}\right)$; HRMS (ESI positive, $\mathrm{m} / \mathrm{z}$ ): calculated for $\mathrm{C}_{42} \mathrm{H}_{51} \mathrm{~N}_{7} \mathrm{O}_{6} \mathrm{~S}$ 781.3622; found $781.3643(2.72 \mathrm{ppm})$

tert-Butyl ((1-(4-(4-(2-([1,1'-biphenyl]-4-yl)ethyl)thiazol-2-yl)-3-(2-(2-oxoimidazolidin-1yl)ethoxy)phenyl)-1H-imidazol-2-yl)methyl)(2-((tert-

butoxycarbonyl)amino)ethyl)carbamate (37f). Yield: $80 \%$ \%. ${ }^{1} \mathrm{H}$ NMR (DMSO- $\mathrm{d}_{6}, 400$ $\left.\mathrm{MHz}, 80^{\circ} \mathrm{C}\right) \delta(\mathrm{ppm}): 8.39(\mathrm{~d}, J=8.3 \mathrm{~Hz}, 1 \mathrm{H}), 7.67-7.60(\mathrm{~m}, 2 \mathrm{H}), 7.56(\mathrm{~d}, J=8.2 \mathrm{~Hz}$, 2H), $7.48-7.40(\mathrm{~m}, 2 \mathrm{H}), 7.38-7.29(\mathrm{~m}, 6 \mathrm{H}), 7.11(\mathrm{dd}, J=8.4,2.0 \mathrm{~Hz}, 1 \mathrm{H}), 7.03(\mathrm{~d}, J=$ $1.4 \mathrm{~Hz}, 1 \mathrm{H}), 6.54$ (bs, 1H, NHBoc), 6.06 (bs, 1H, NCONH), $4.55\left(\mathrm{~s}, 2 \mathrm{H}, \mathrm{ImCH}_{2} \mathrm{~N}\right), 4.42$ (t, $\left.J=5.8 \mathrm{~Hz}, 2 \mathrm{H}, \mathrm{OC}_{2} \mathrm{CH}_{2}\right), 3.61\left(\mathrm{t}, J=5.8 \mathrm{~Hz}, 2 \mathrm{H}, \mathrm{OCH}_{2} \mathrm{CH}_{2}\right), 3.48(\mathrm{dd}, J=8.9,6.6 \mathrm{~Hz}$, $2 \mathrm{H}, \mathrm{C}_{2} \mathrm{CH}_{2} \mathrm{NHCON}$ ), 3.26 (dd, $J=8.5,6.3 \mathrm{~Hz}, 4 \mathrm{H}, \mathrm{CH}_{2} \mathrm{C}_{2} \mathrm{NHCON}_{2} \mathrm{CH}_{2} \mathrm{CH}_{2} \mathrm{NHBoc}_{\text {), }}$ 3.18 - 3.02 (m, $\left.6 \mathrm{H}, \mathrm{C}_{2} \mathrm{CH}_{2} \mathrm{NHBoc}, \mathrm{C}_{2} \mathrm{C}_{2} \mathrm{BiPh}\right), 1.37$ (s, 9H, $\left.\mathrm{CH}_{3}\right), 1.26$ (s, 9H, $\left.\mathrm{CH}_{3}\right)$; ${ }^{13} \mathrm{C}$ NMR (DMSO-d $\left.\mathrm{d}_{6}, 100 \mathrm{MHz}, 80^{\circ} \mathrm{C}\right) \delta(\mathrm{ppm}): 161.5(\mathrm{NHCON}), 159.3\left(\mathrm{C}_{\mathrm{Ar}}\right), 155.0$ (NHCOO), 154.9 (NHCOO), $154.0\left(\mathrm{C}_{\mathrm{Ar}}\right), 144.1\left(\mathrm{C}_{\mathrm{Ar}}\right), 140.3\left(\mathrm{C}_{\mathrm{Ar}}\right), 139.8\left(\mathrm{C}_{\mathrm{Ar}}\right), 138.2$ 
$\left(\mathrm{C}_{\mathrm{Ar}}\right), 137.5\left(\mathrm{CH}_{\mathrm{Ar}}\right), 128.4\left(\mathrm{CH}_{\mathrm{Ar}}\right), 128.3\left(\mathrm{CH}_{\mathrm{Ar}}\right), 128.3\left(\mathrm{CH}_{\mathrm{Ar}}\right), 127.1\left(\mathrm{CH}_{\mathrm{Ar}}\right), 126.6\left(\mathrm{C}_{\mathrm{Ar}}\right)$, $126.0\left(\mathrm{CH}_{\mathrm{Ar}}\right), 126.0\left(\mathrm{CH}_{\mathrm{Ar}}\right), 121.6\left(\mathrm{CH}_{\mathrm{Im}}\right), 121.2\left(\mathrm{CH}_{\mathrm{Im}}\right), 117.5\left(\mathrm{CH}_{\mathrm{Ar}}\right), 115.2\left(\mathrm{CH}_{\mathrm{Ar}}\right)$,

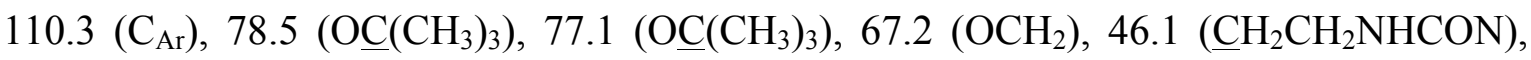
$44.9\left(\mathrm{OCH}_{2} \mathrm{CH}_{2}\right), 42.8\left(\mathrm{ImCH}_{2} \mathrm{~N}\right), 42.2\left(\mathrm{CH}_{2} \mathrm{CH}_{2} \mathrm{NHBoc}\right), 38.0\left(\mathrm{CH}_{2} \mathrm{CH}_{2} \mathrm{NHCON}\right), 37.1$ $\left(\mathrm{CH}_{2} \mathrm{CH}_{2} \mathrm{NHBoc}\right), 33.9$ (ThiazCH $\left.\mathrm{CH}_{2}\right), 32.0\left(\mathrm{ThiazCH}_{2} \mathrm{CH}_{2}\right), 27.8\left(\mathrm{CH}_{3}\right), 27.5\left(\mathrm{CH}_{3}\right)$; HRMS (ESI positive, $m / z$ ): calculated for $\mathrm{C}_{44} \mathrm{H}_{53} \mathrm{~N}_{7} \mathrm{O}_{6} \mathrm{~S} \quad 807.3778$; found 807.3804 (3.2 ppm)

\section{REFERENCES:}

(1) Ismail, M. A., Anbazhagan, M., Stephens, C. E., Boykin, D. W. (2004) Synthesis of benzyloxybromobenzonitriles. Synth. Commun. 34, 751-758 DOI 10.1081/SCC-120028347. (2) Zav'yalov, S. I., Kravchenko, N. E. (1984) Effect of amides on the bromination of methyl alkyl ketones. Izvestiya Akademii Nauk SSSR, Seriya Khimicheskaya. 12, 2756-2759.

(3) Barker, J. J., Barker, O., Boggio, R., Chauhan, V., Cheng, R. K. Y., Corden, V., Courtney, S. M., Edwards, N., Falque, V. M., Fusar, F., Gardiner, M., Hamelin, E., Hesterkamp, T., Ichihara, O., Jones, R. S., Mather, O., Mercurio, C., Minucci, S., Montalbetti, C., Muller, A., Patel, D., Phillips, B. G., Varasi, M., Whittaker, M., Winkler, D., Yarnold, C. J. (2009) Fragment-based identification of Hsp90 inhibitors. ChemMedChem. 4, 963-966. DOI: 10.1002/cmdc.200900011

(4) Ngo, Q. A., Nguyen, L. A., Vo, N. B., Nguyen, T. H., Rous, F., Nguyen, T. H., Nguyen, V. T. (2015) Synthesis and antiproliferative activity of new vinca alkaloids containing an $\alpha, \beta$-unsaturated aromatic side chain. Bioorg. Med. Chem. Lett. 25, 5597-5600. DOI: $\underline{10.1016 / \mathrm{j} . \mathrm{bmcl} \cdot 2015.10 .040}$ 
Table S1. Experimental water solubility and other physicochemical parameters of imidazo-phenyl-thiazole compounds 3a-f

\begin{tabular}{|c|c|c|c|c|}
\hline Comp & $\mathrm{S}(\mathrm{mg} / \mathrm{mL})^{\mathrm{a}}$ & $\log S$ & $\operatorname{cLog} P^{b}$ & MW \\
\hline $3 \mathbf{a}$ & 170 & -0.70 & -3.2 & 681.7 \\
\hline $3 b$ & $>25$ & $>-1.5$ & -2.6 & 695.7 \\
\hline $3 c$ & - & - & -2.8 & 715.7 \\
\hline 3d & 97 & -0.97 & -2.4 & 729.7 \\
\hline $3 e$ & 57 & -1.19 & -1.2 & 779.8 \\
\hline $3 f$ & 84 & -1.08 & -0.7 & 805.8 \\
\hline
\end{tabular}

${ }^{\mathrm{a}}$ Thermodynamic water solubilities measure at $25{ }^{\circ} \mathrm{C}$ after $24 \mathrm{~h}$. Values are typically means of three measurements

b $\operatorname{LogP}$ values were estimated by using OSIRIS property explorer (https://www.organicchemistry.org/prog/peo/)

Figure S1. Absorbance and emission spectra of compound 3d in acetonitrile.

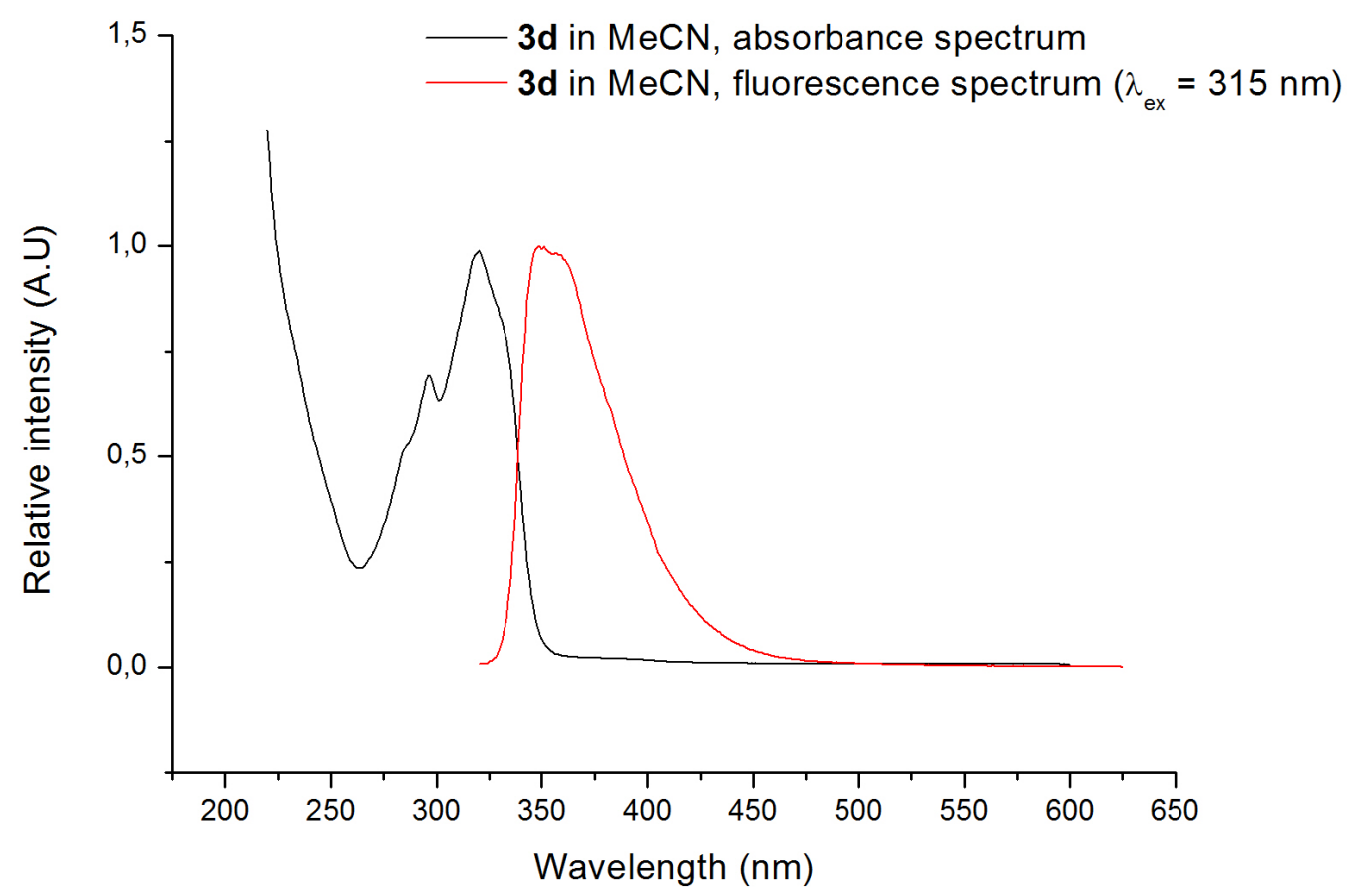


Table S2: Crystallographic data for $L i$ TryR:2f complex* .

\begin{tabular}{|c|c|}
\hline & $L i$-TryR:2f complex \\
\hline \multicolumn{2}{|c|}{ Data Collection Statistics } \\
\hline Wavelength $(\AA)$ & 0.979260 \\
\hline Space group & P $2{ }_{1} 2_{1} 2_{1}$ \\
\hline$a, b, c(\AA)$ & $94.18,106.39,186.55$ \\
\hline$\alpha, \beta, g\left({ }^{\circ}\right)$ & $90,90,90$ \\
\hline Resolution range $(\AA)$ & $\begin{array}{c}46.64-3.30(3.56- \\
3.30)\end{array}$ \\
\hline Total reflections & $78272(11212)$ \\
\hline Unique reflections & $23420(4304)$ \\
\hline Completeness (\%) & $81.7(74)$ \\
\hline Multiplicity & $3.3(2.6)$ \\
\hline $\mathrm{CC} 1 / 2$ & $0.99(0.79)$ \\
\hline $\mathrm{R}_{\text {pim }}$ & $0.07(0.39)$ \\
\hline Avg. I/ $\sigma(\mathrm{I})$ & $5.2(1.3)$ \\
\hline \multicolumn{2}{|l|}{ Refinement Statistics } \\
\hline Resolution range $(\AA)$ & $46.64-3.30$ \\
\hline $\mathrm{R}_{\mathrm{work}} / \mathrm{R}_{\text {free }}$ & $0.24 / 0.27$ \\
\hline \multicolumn{2}{|l|}{ No. atoms } \\
\hline Protein & 7403 \\
\hline Water & 195 \\
\hline Ligand & 170 \\
\hline Bond length $(\AA)$ & 0.0075 \\
\hline Bond angles $\left({ }^{\circ}\right)$ & 1.61 \\
\hline $\begin{array}{l}\text { Ramachandran } \\
\text { favored/outliers (\%) }\end{array}$ & $99.5 / 0.5$ \\
\hline Residues in the AU & 978 \\
\hline PDB code & $617 \mathrm{~N}$ \\
\hline
\end{tabular}

*Values between parentheses correspond to the highest resolution shells 
Figure S2: Electron density for $\mathbf{2 f}$ ligand

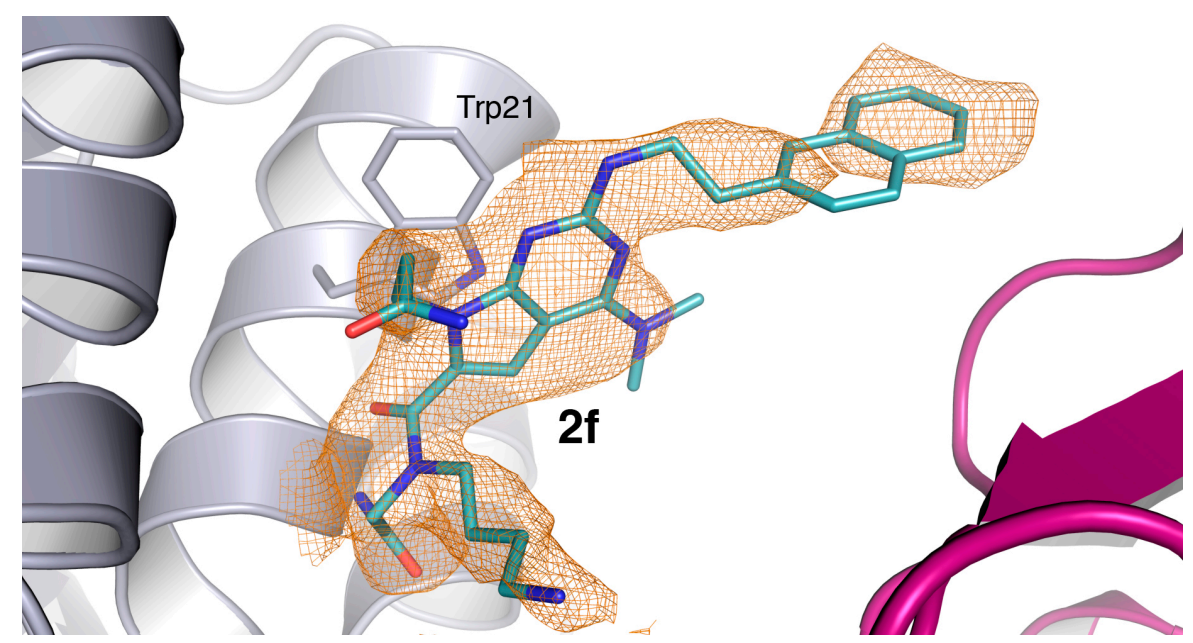

$2 \mathrm{~F}_{\mathrm{o}}-\mathrm{F}_{\mathrm{c}}$ electron density map (orange mesh) contoured at $1 \sigma$ for $\mathbf{2 f}$ (stick representation with $\mathrm{C}$ atoms colored in cyan) in the LiTryR:2f complex. $L i$-TryR is shown as a cartoon, with each monomer coloured differently. Side chain of residue Trp21 is represented as capped sticks and labeled.

Figure S3: Structural changes in $L i$-TryR upon $2 \mathbf{f}$ binding

A

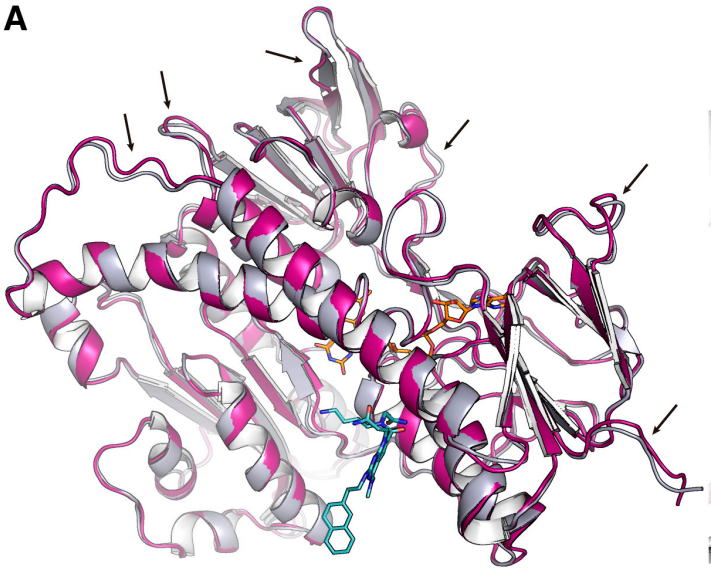

B

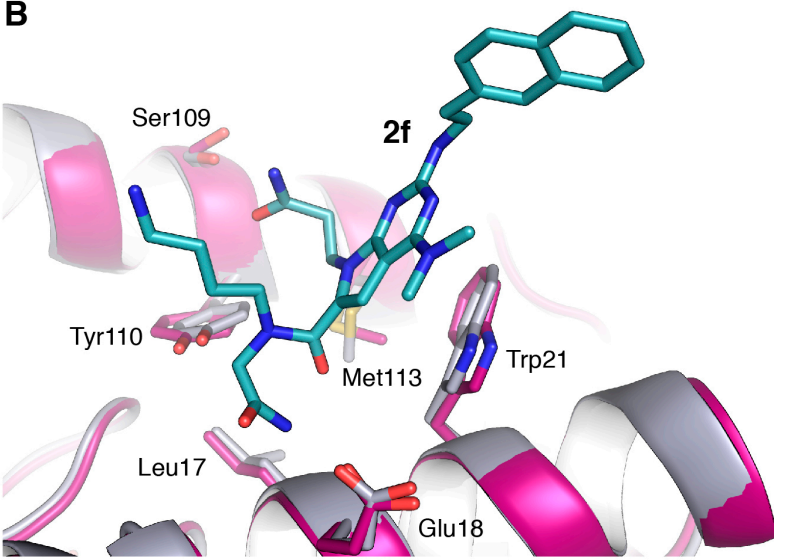

(A) Structural superimposition of chain A (pink) and chain B (gray) of the Li-TryR:2f complex. Main changes in the backbone between both chains are highlighted with arrows. (B) Upon 2 f (cyan sticks) binding on chain B of $L i$-TryR, changes are observed in the orientation of side chains of the residues stabilizing the ligand. Relevant residues are depicted as capped sticks and have been labeled for reference. 
Figure S4. Comparison of active site occupancy in $L i$-TryR by $\mathbf{2 f}$ and other $T b$-TryR described inhibitors

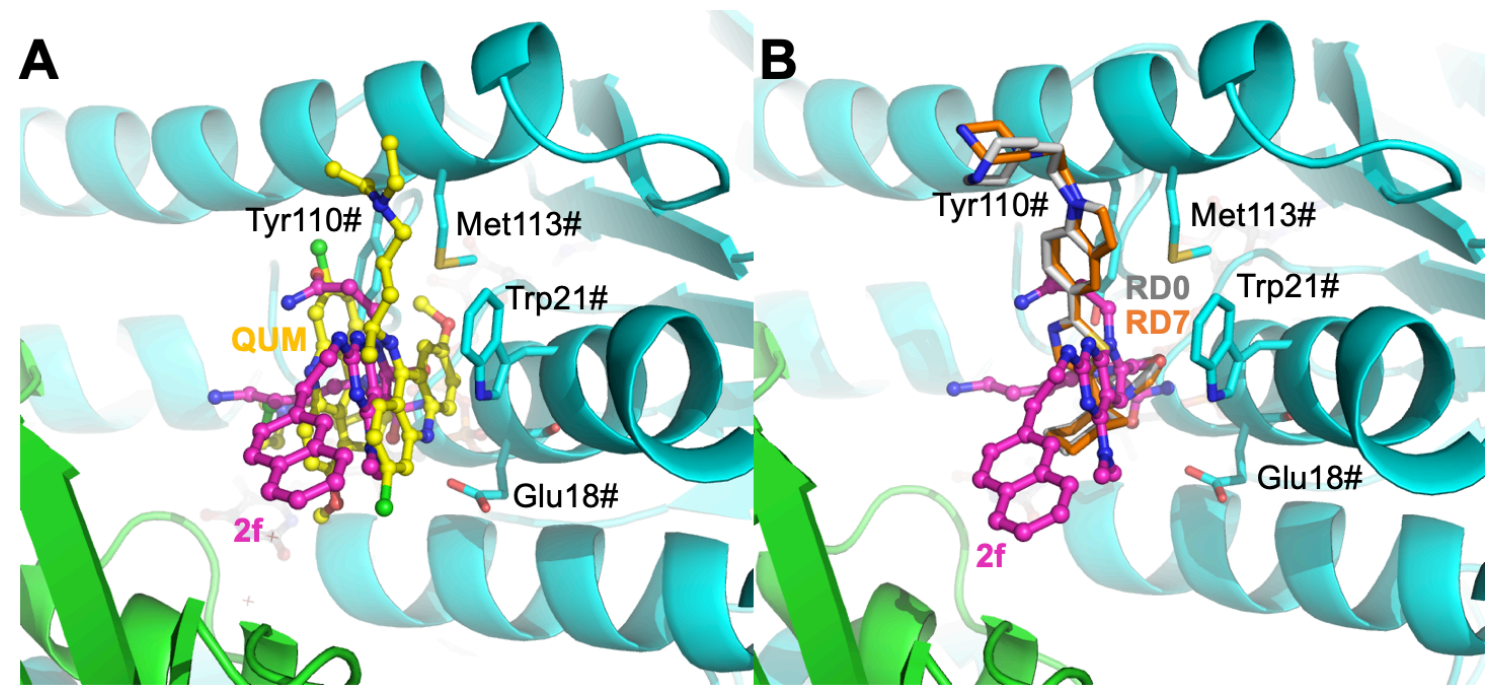

Comparison of the $L i$-TryR:2f complex (ligand $\mathrm{C}$ atoms colored in magenta, PDB id. $6 \mathrm{I7N})$ with $T b$-TryR in complex with other described inhibitors upon best-fit $\mathrm{C} \alpha$ superimposition of the highly homologous proteins. For simplicity, only $L i$-TryR is shown, with chains A and B colored in green and cyan, respectively. A. Comparison with the covalent adduct of quinacrine mustard (ligand $\mathrm{C}$ atoms colored in yellow) with $T b$-TryR (PDB id. 1GXF). B. Comparison with the two cyclohexylpyrrolidine inhibitors RD0 (PDB id. 6BTL, ligand $\mathrm{C}$ atoms colored in grey) and $\mathrm{RD} 7$ (PDB id. 6BTU, ligand $\mathrm{C}$ atoms colored in orange) bound to $T b$-TryR. ${ }^{1}$ 
Figure S5. Pharmacophore model for the series 2 compounds.

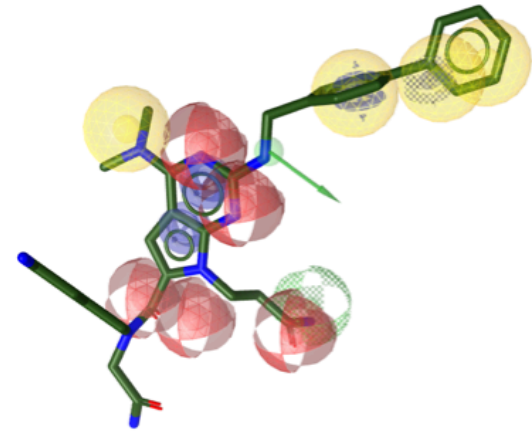

2g (active)

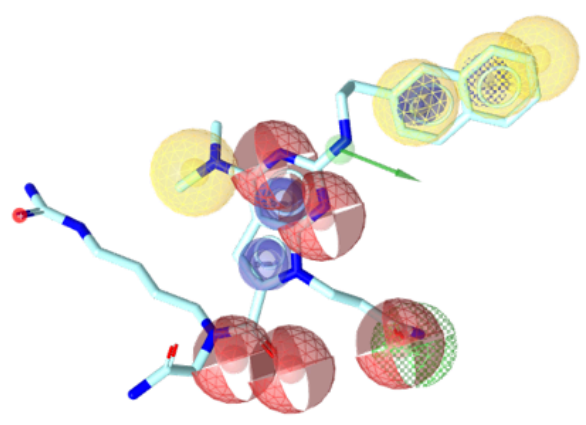

2i

(active)

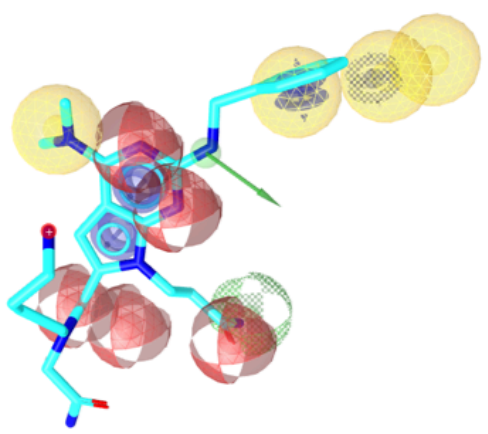

2c (inactive)

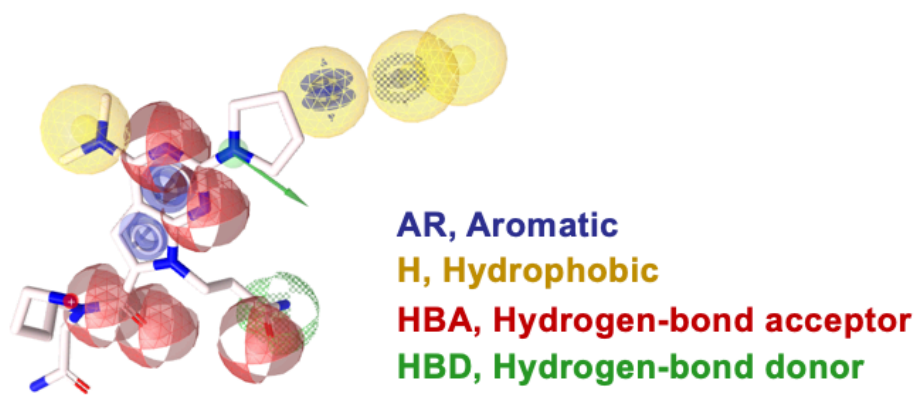

2h

(inactive)

Superimposition of four representative compounds onto the 3D pharmacophore model, which consists of four features: aromatic (AR), hydrophobic $(\mathrm{H})$, hydrogen-bond acceptor (HBA) and hydrogen-bond donor (HBD). Active compounds $2 \mathbf{g}(\mathrm{C}$ atoms in green) and $\mathbf{2} \mathbf{i}(\mathrm{C}$ atoms in turquoise) cover the $\mathrm{H}+\mathrm{AR}$ region on the top right-hand corner of each figure whereas inactive compounds $\mathbf{2 c}$ (C atoms in cyan) and $\mathbf{2 h}$ ( $\mathrm{C}$ atoms in light grey) do not. 
Figure S6. Calculated binding energies $\left(\mathrm{kcal} \mathrm{mol}^{-1}\right)$ per residue and component contributions (Table below) for the binding of $\mathbf{3 f}$ and peptide $\mathbf{1 a}$ to $L i$-TryR monomer. ${ }^{2}$

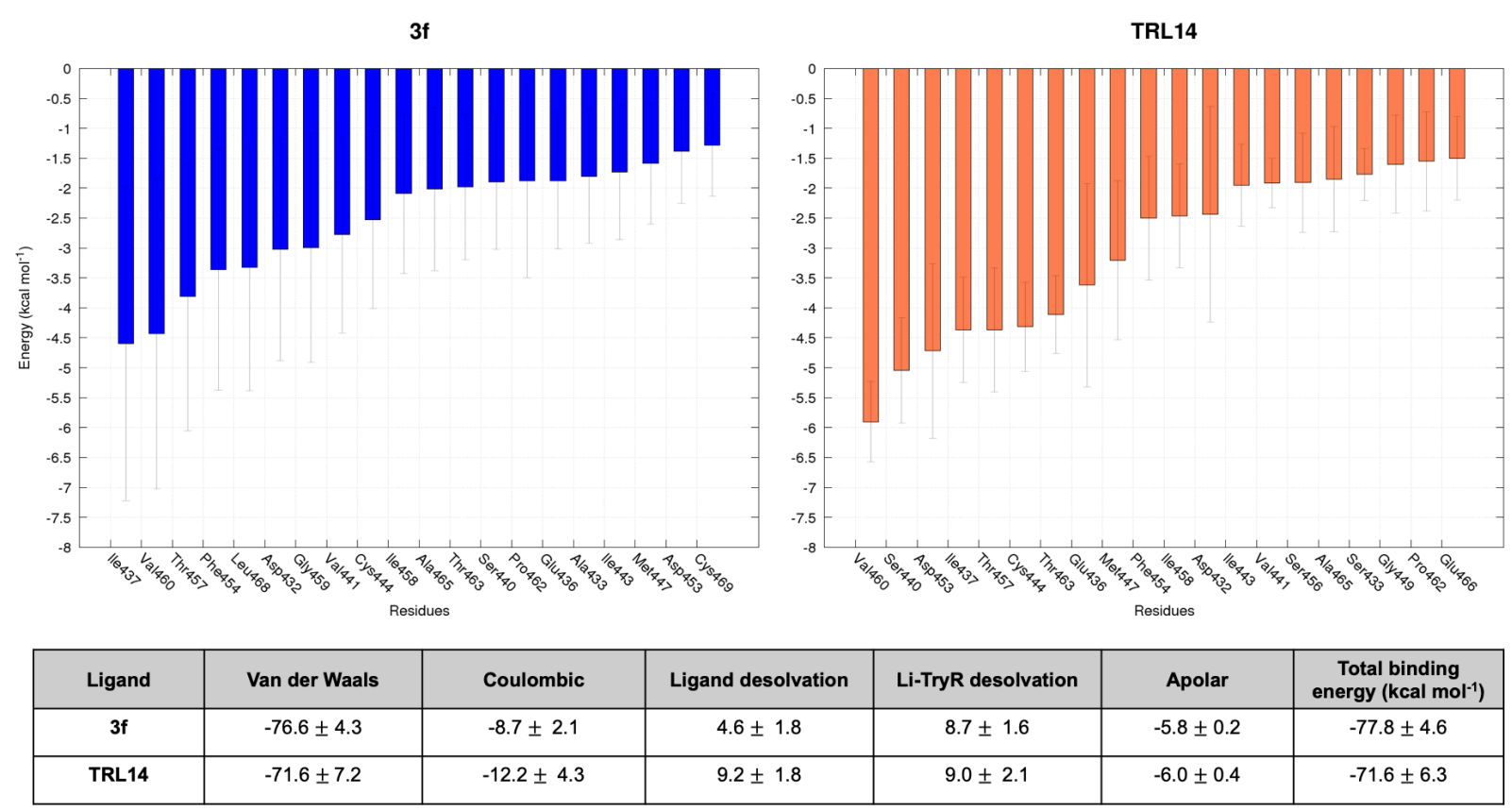

\section{REFERENCES:}

(1) De Gasparo, R., Brodbeck-Persch, E., Bryson, S., Hentzen, N. B., Kaiser, M., Pai, E. F., Krauth-Siegel, R. L., Diederich, F. (2018) Biological evaluation and X-ray co-crystal structures of cyclohexylpyrrolidine ligands for trypanothione reductase, an enzyme from the redox metabolism of Trypanosoma. ChemMedChem 13, 957-967. DOI: 10.1002/cmdc.201800067.

(2) Klett, J., Núñez-Salgado, A., Dos Santos, H. G., Cortés-Cabrera, A., Perona, A., GilRedondo, R., Abia, D., Gago, F., Morreale, A. (2012) MM-ISMSA: an ultrafast and accurate scoring function for protein-protein docking. J. Chem. Theory Comput. 8 (9), 3395-3408 DOI 10.1021/ct300497z. 
${ }^{1} \mathrm{H}$-NMR spectra of pyrrolopyrimidines $2 a-i$

\section{Compound $2 a$}

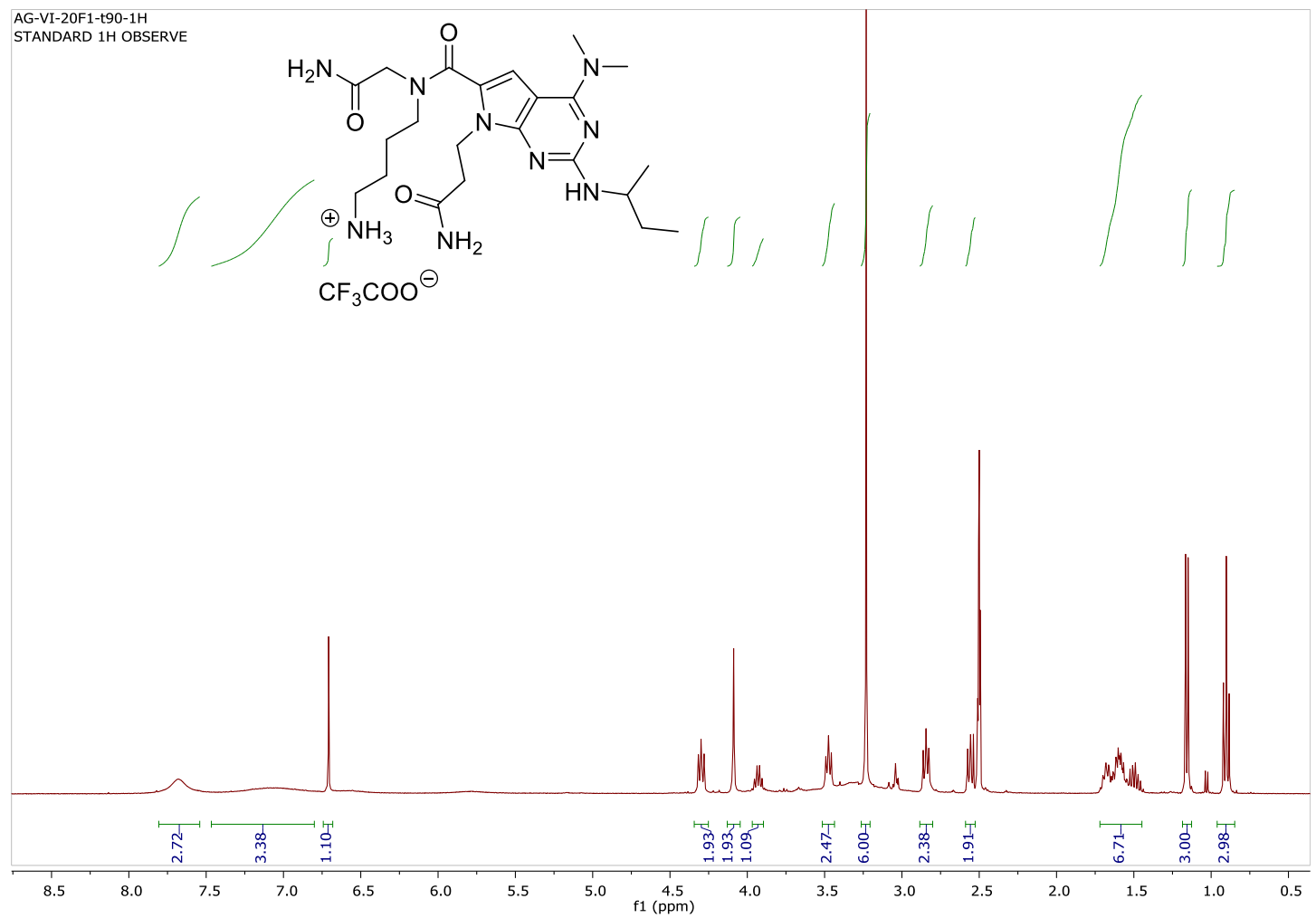

Compound $2 b$ 


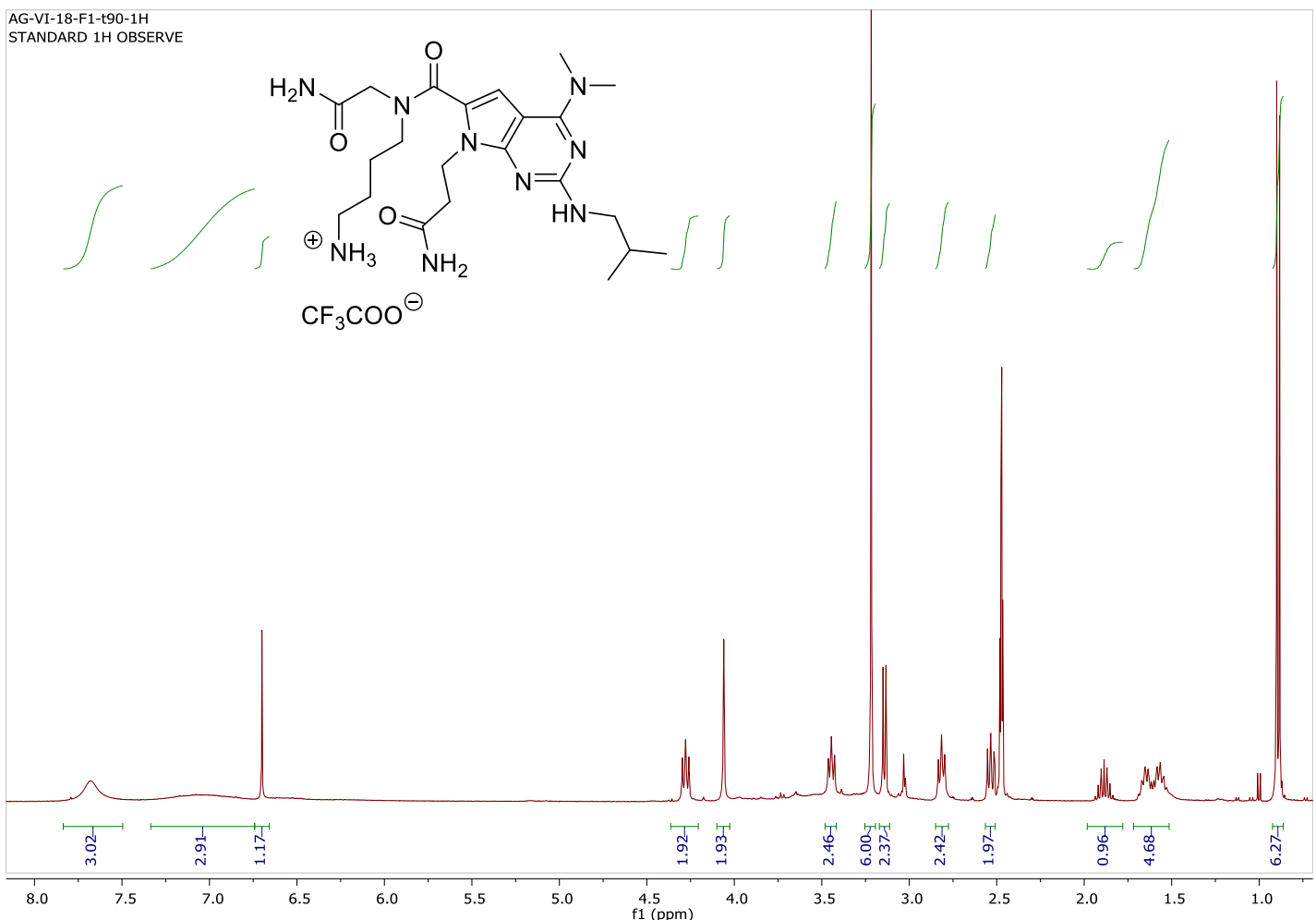

\section{Compound $2 \mathrm{c}$}

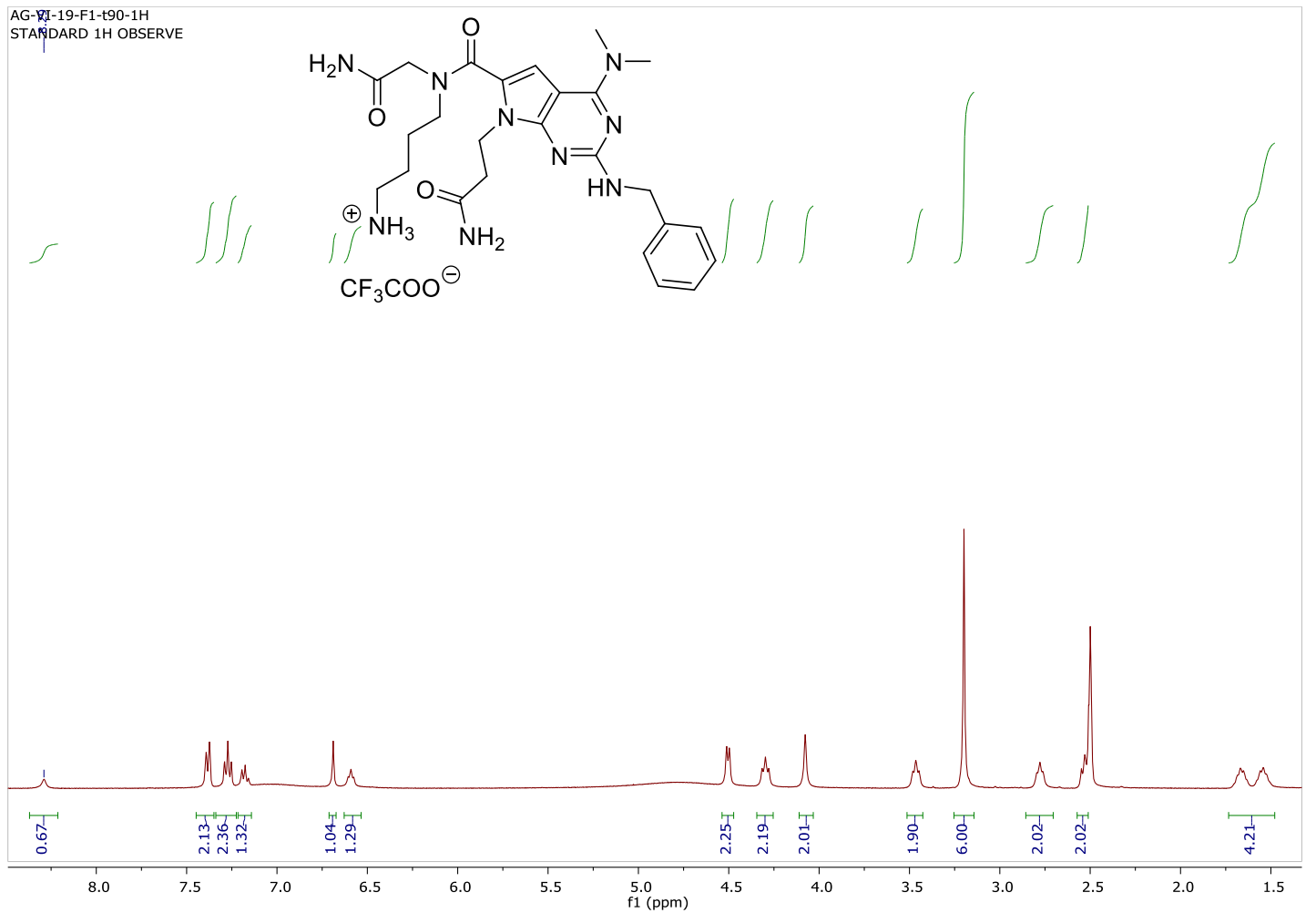

Compound 2d 


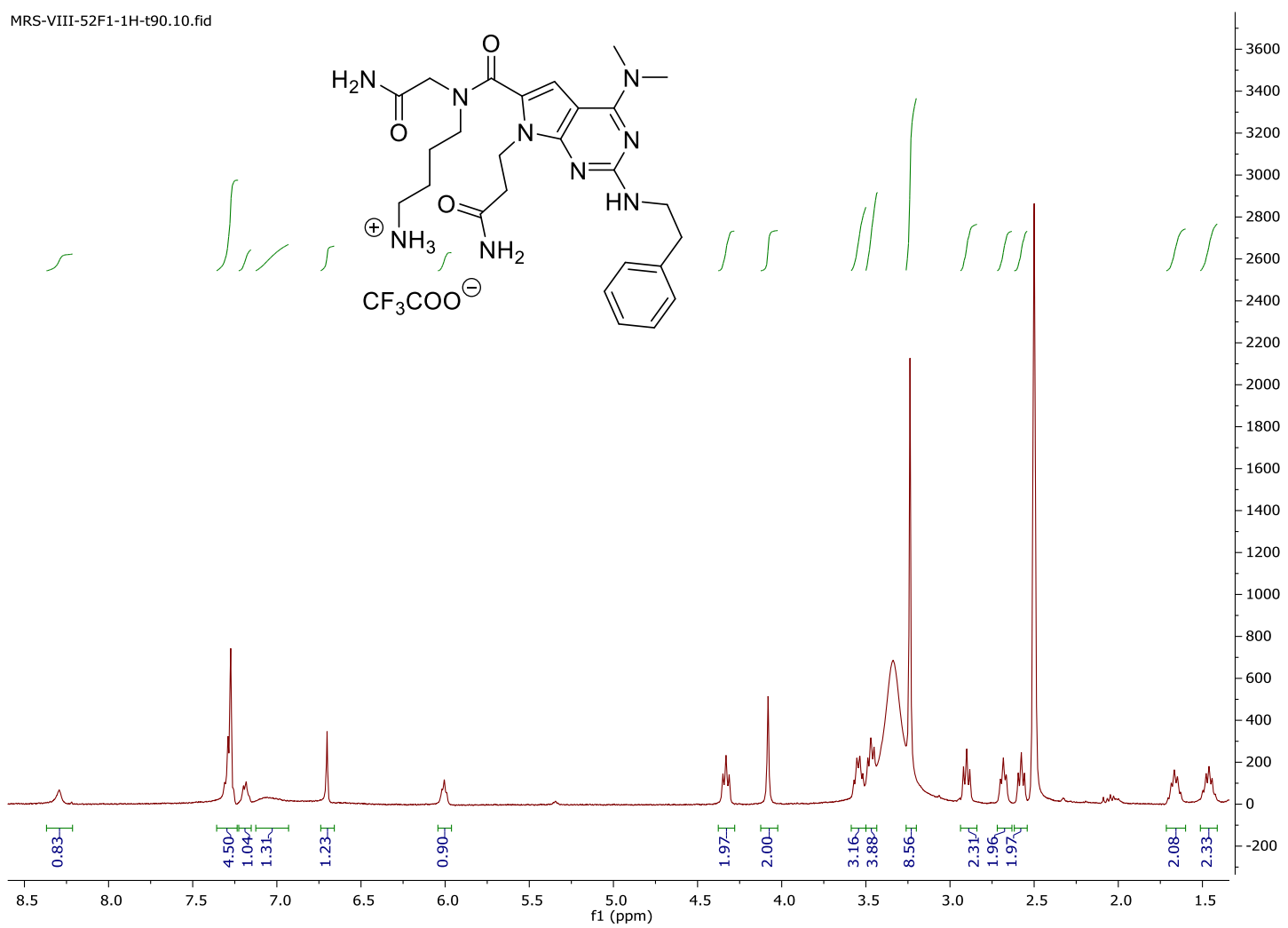

\section{Compound $2 e$}

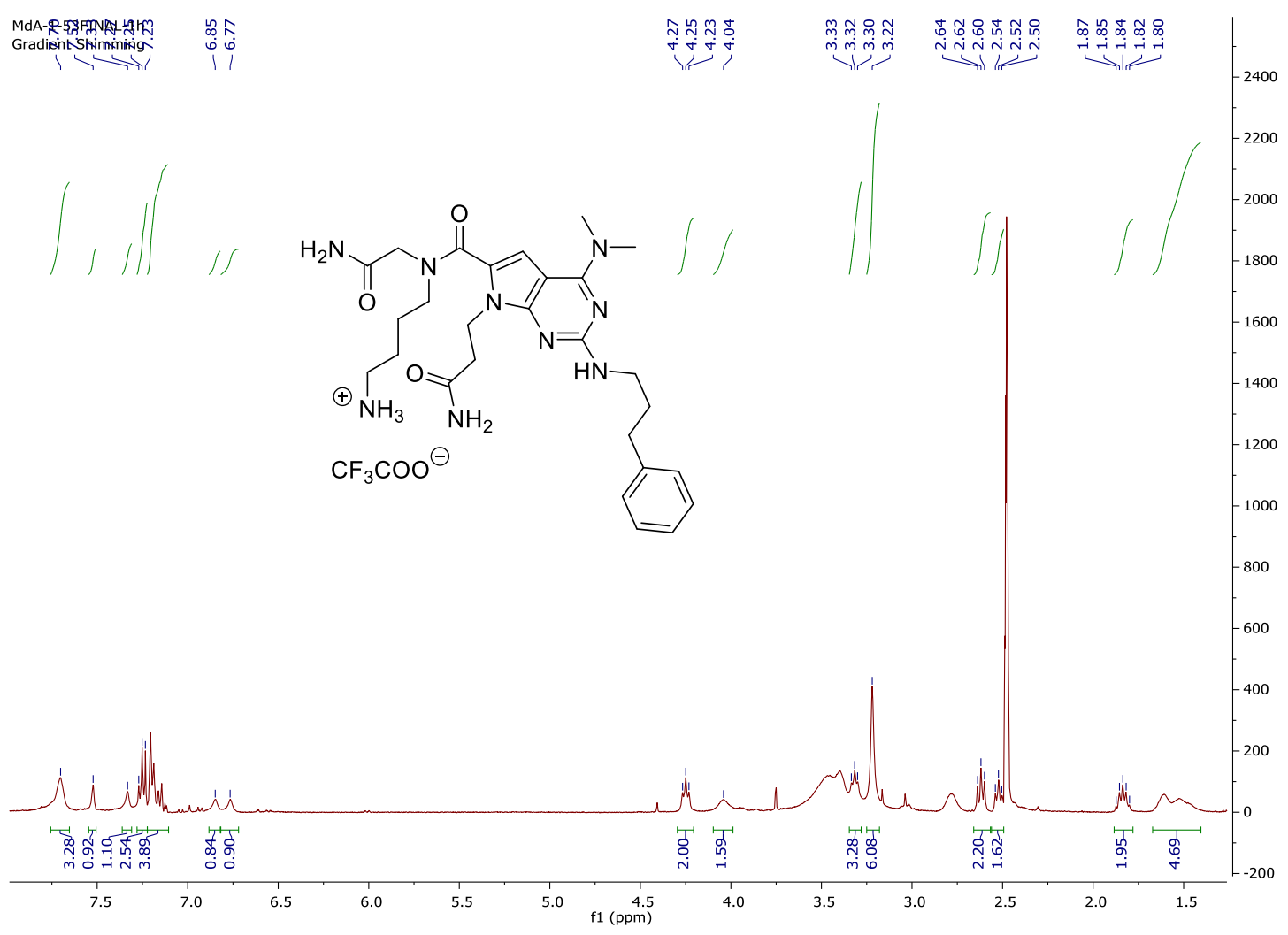

\section{Compound $2 f$}




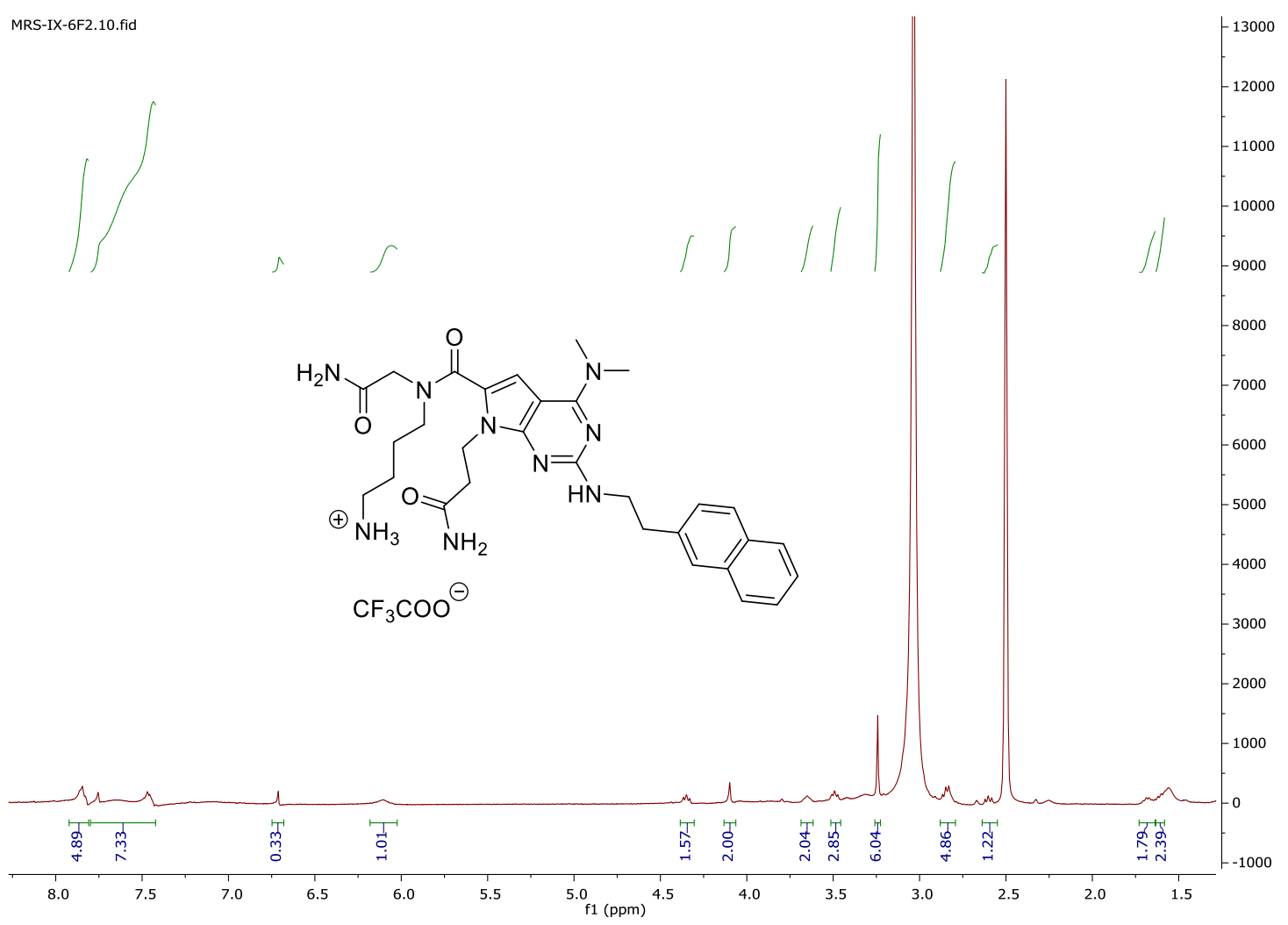

Compound $2 g$

MRS-IX-42T1-t90-1h

STANDARD PROTON PARAMETERS
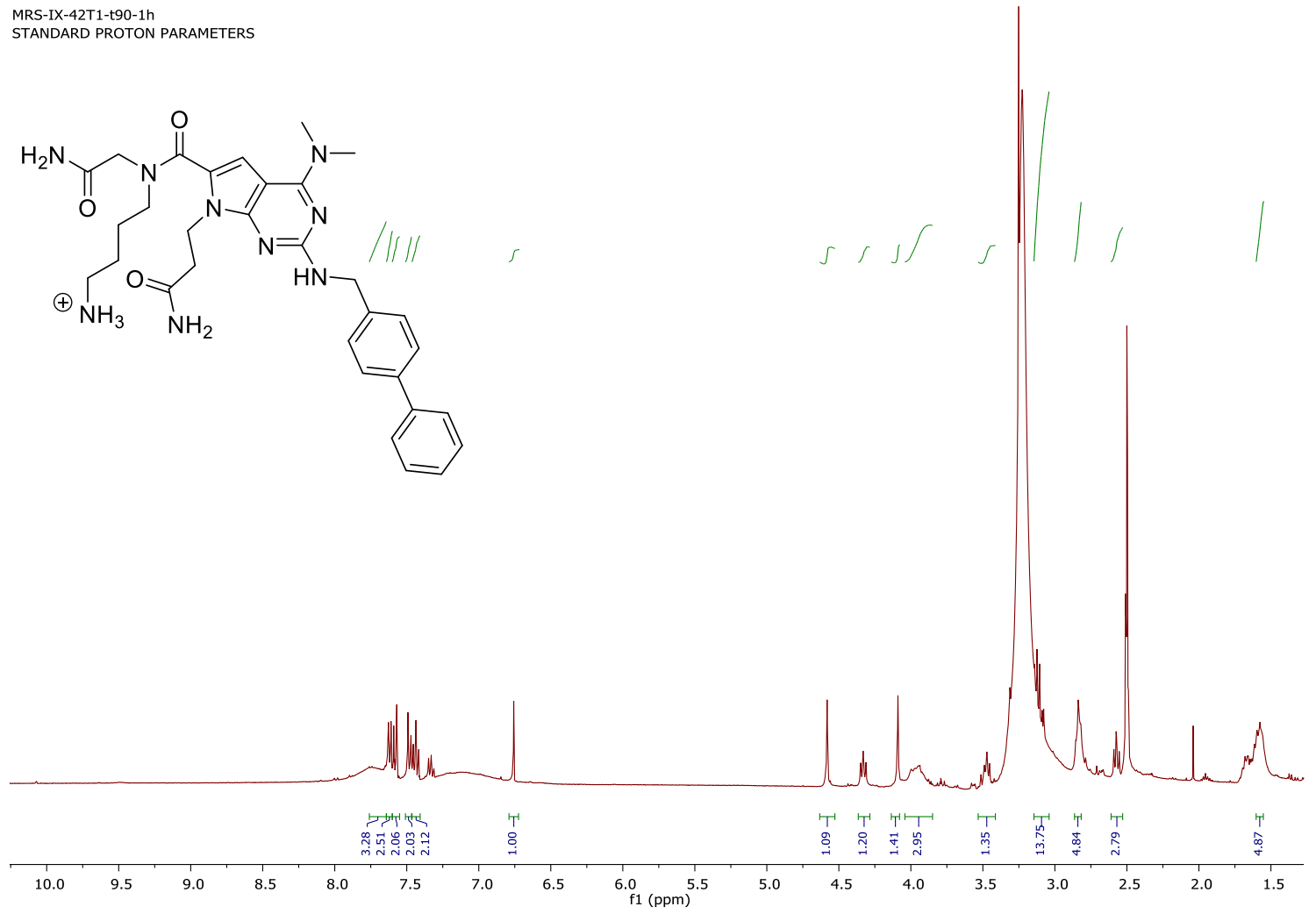

S28 
Compound $2 \mathrm{~h}$

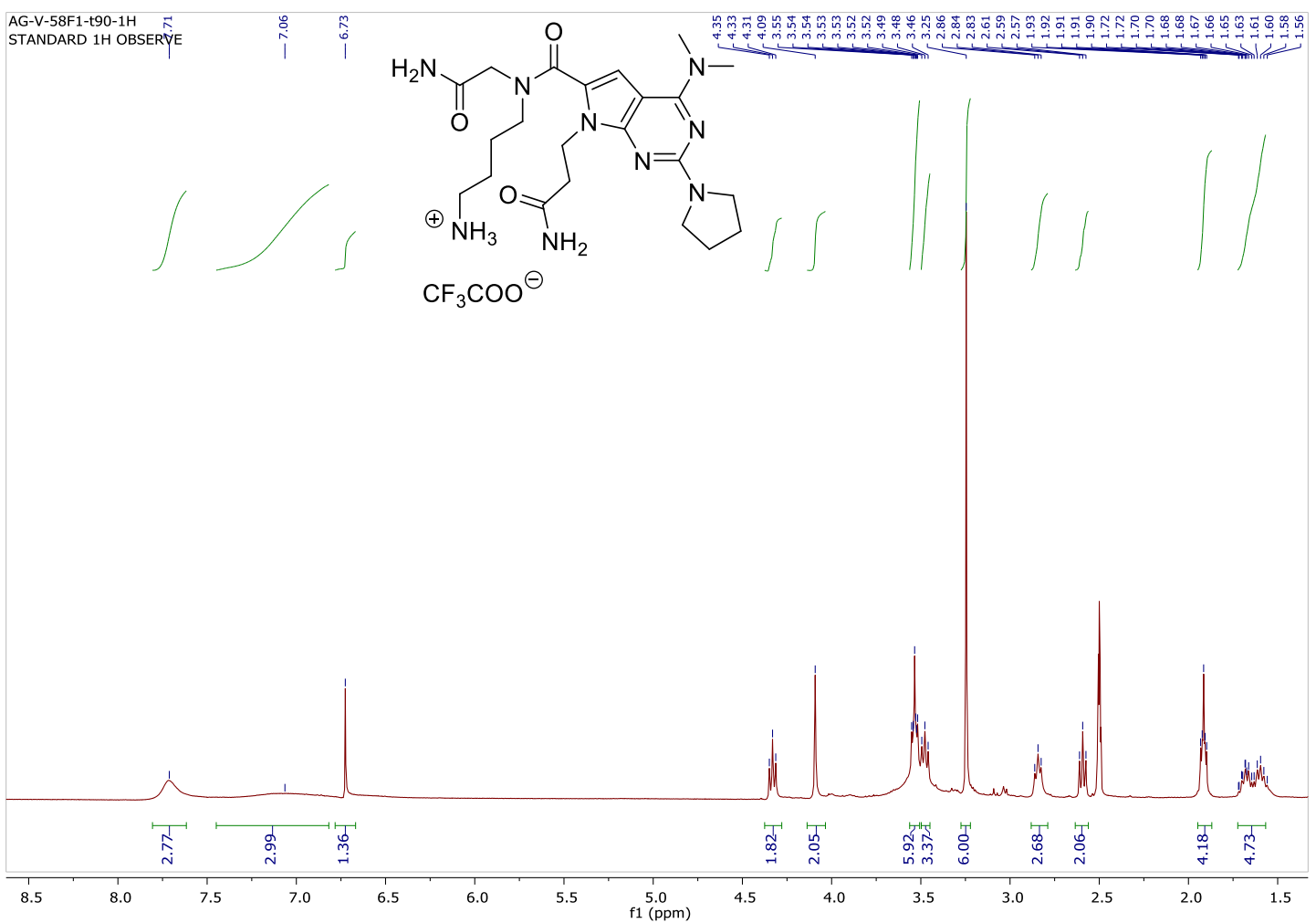

\section{Compound $2 i$}

MRS-IX-45T8-

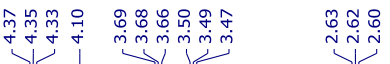

24000

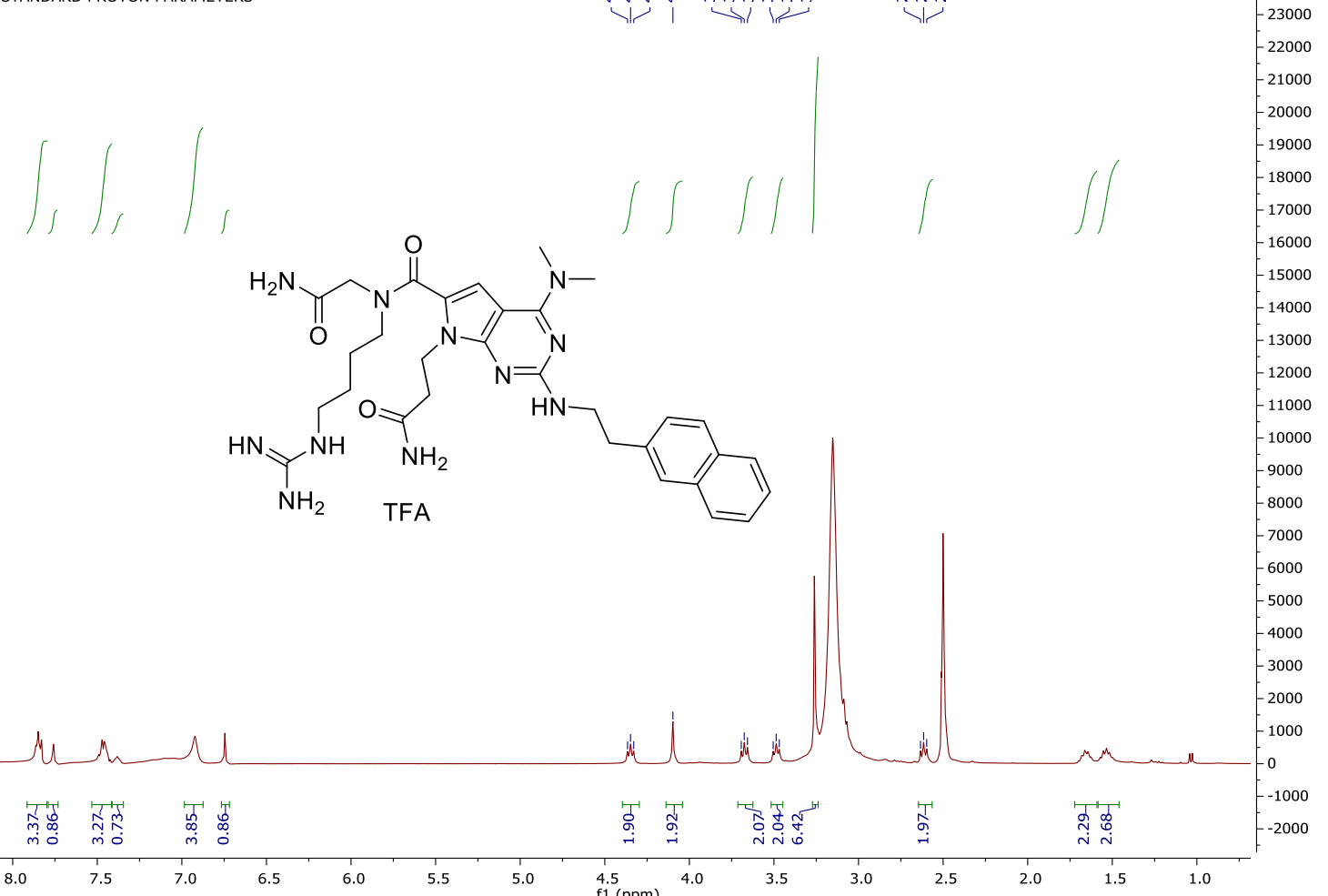


${ }^{1} \mathrm{H}$ NMR spectra of selected intermediates and imidazole-phenyl-thiazoles 3a-f

Compound 26

ARP-II-59ColF15-27

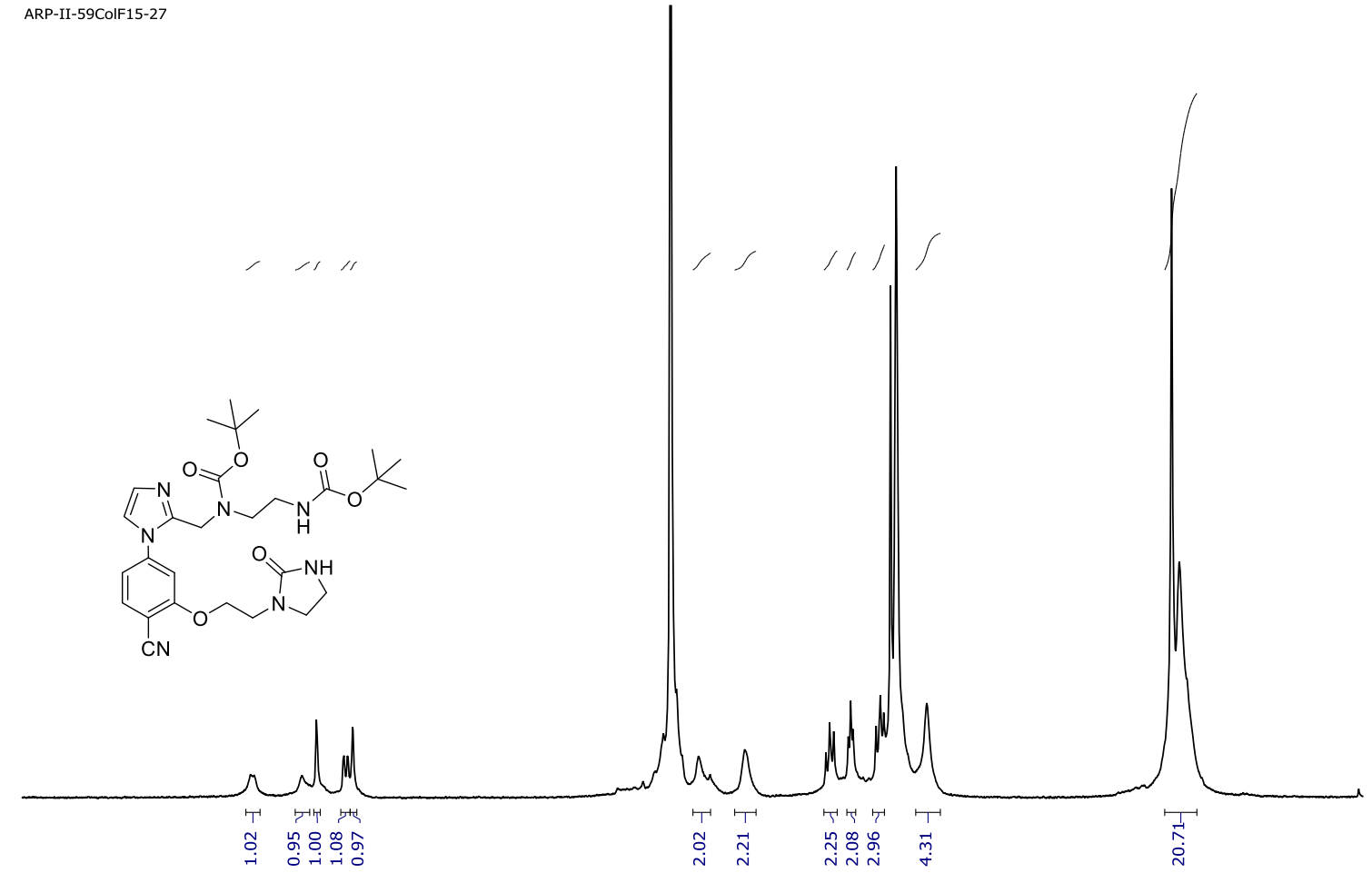

$\begin{array}{rrrrrrrrr}9.0 & 8.5 & 8.0 & 7.5 & 7.0 & 6.5 & 6.0 & 5.5 & \underset{f 1}{1}(\mathrm{ppm})\end{array}$

Compound 27

ARP-III-9-t30<smiles>CC(C)(C)OC(=O)NCCN(Cc1nccn1-c1ccc(C(N)=S)c(OCCN2CCNC2=O)c1)C(=O)OC(C)(C)C</smiles>

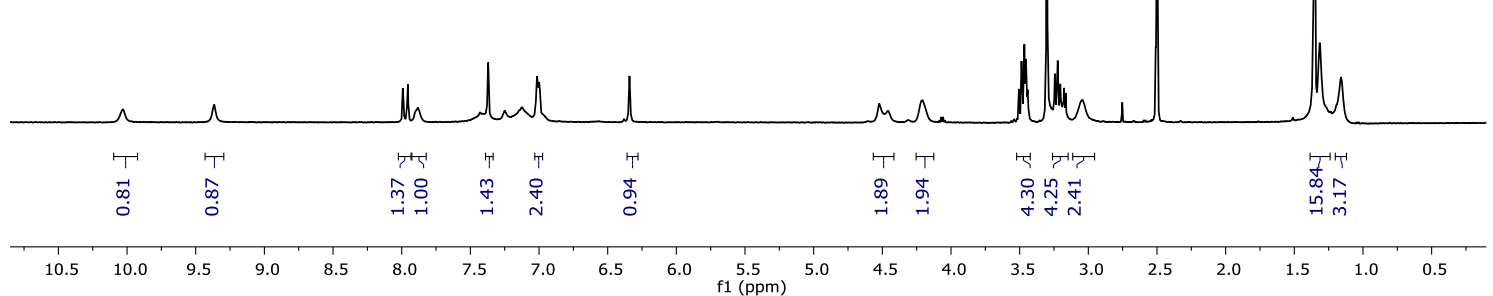




\section{Compound $36 a$}

ARP-III-36ColF7-17

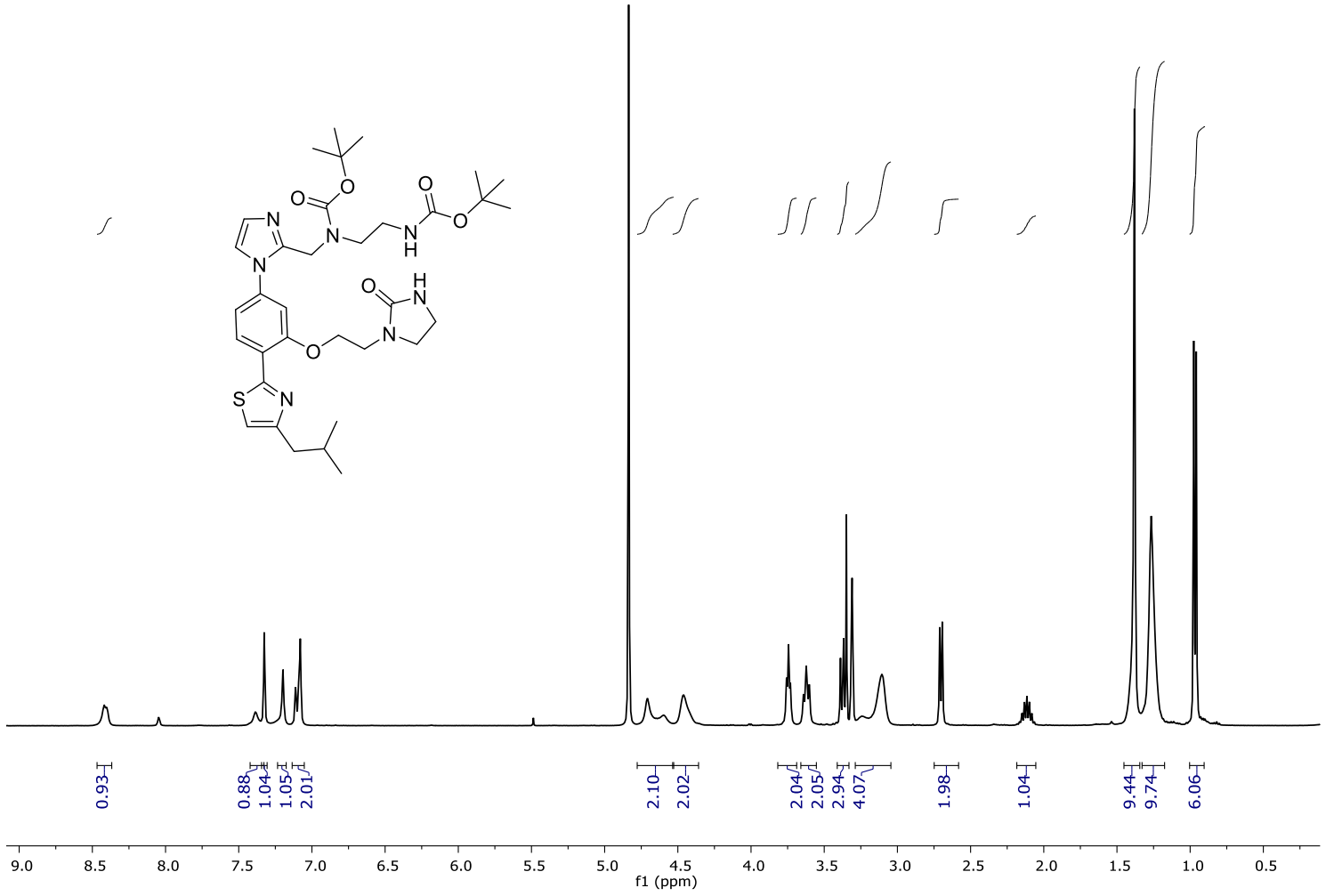

\section{Compound $36 \mathrm{~b}$}

ARP-III-38ColF12-22

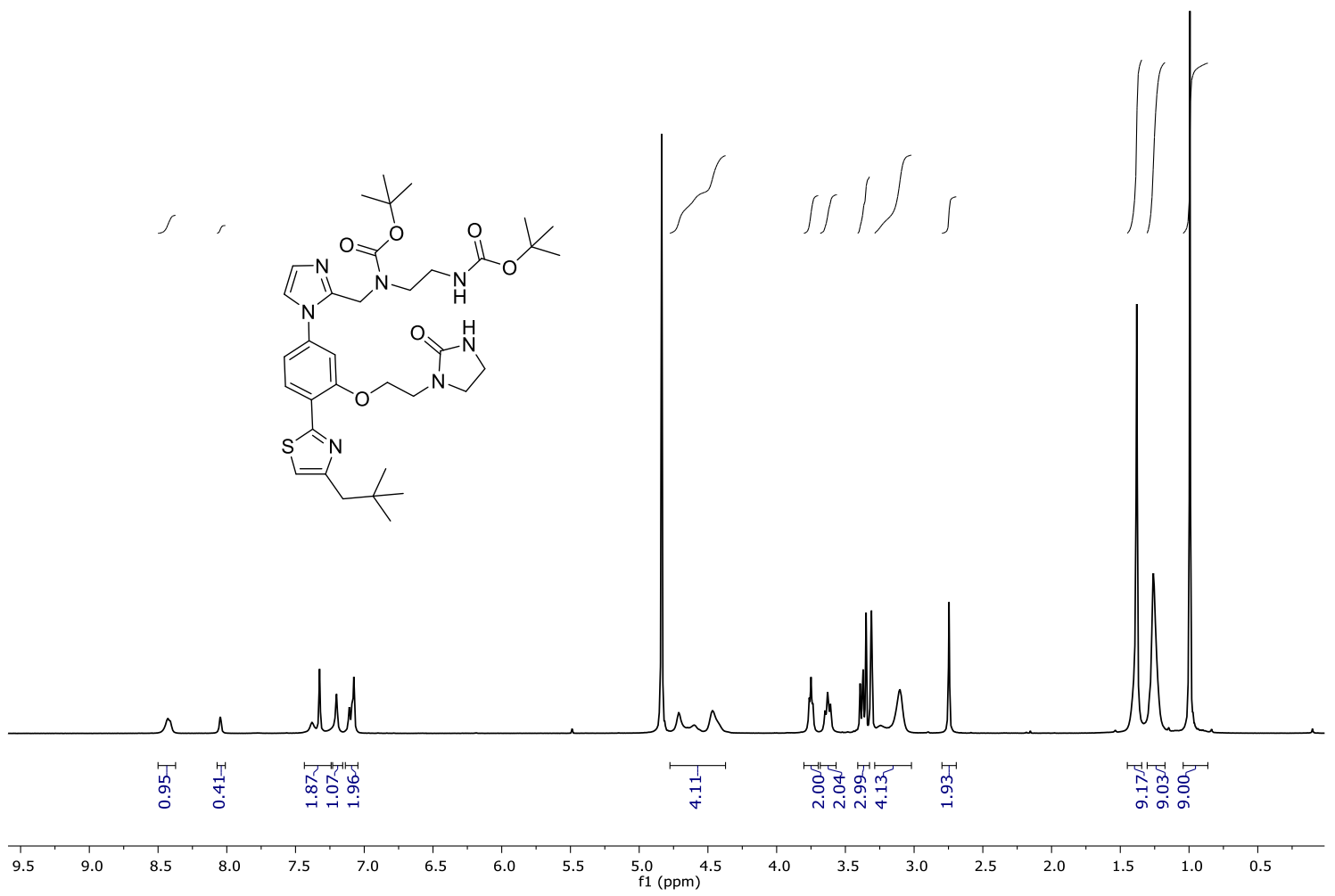




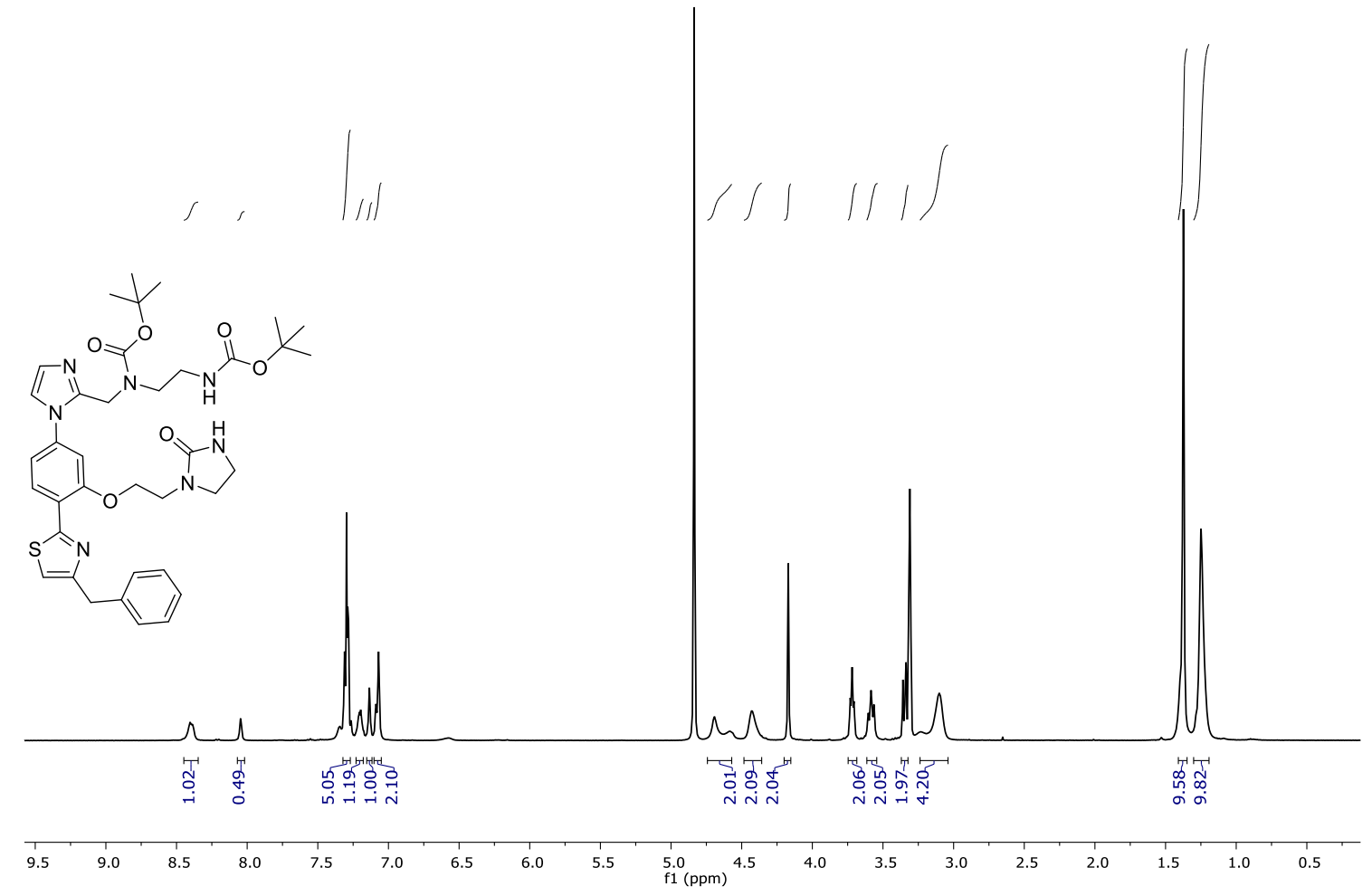

\section{Compound 36d}

ARP-III-48ColF10-22conc

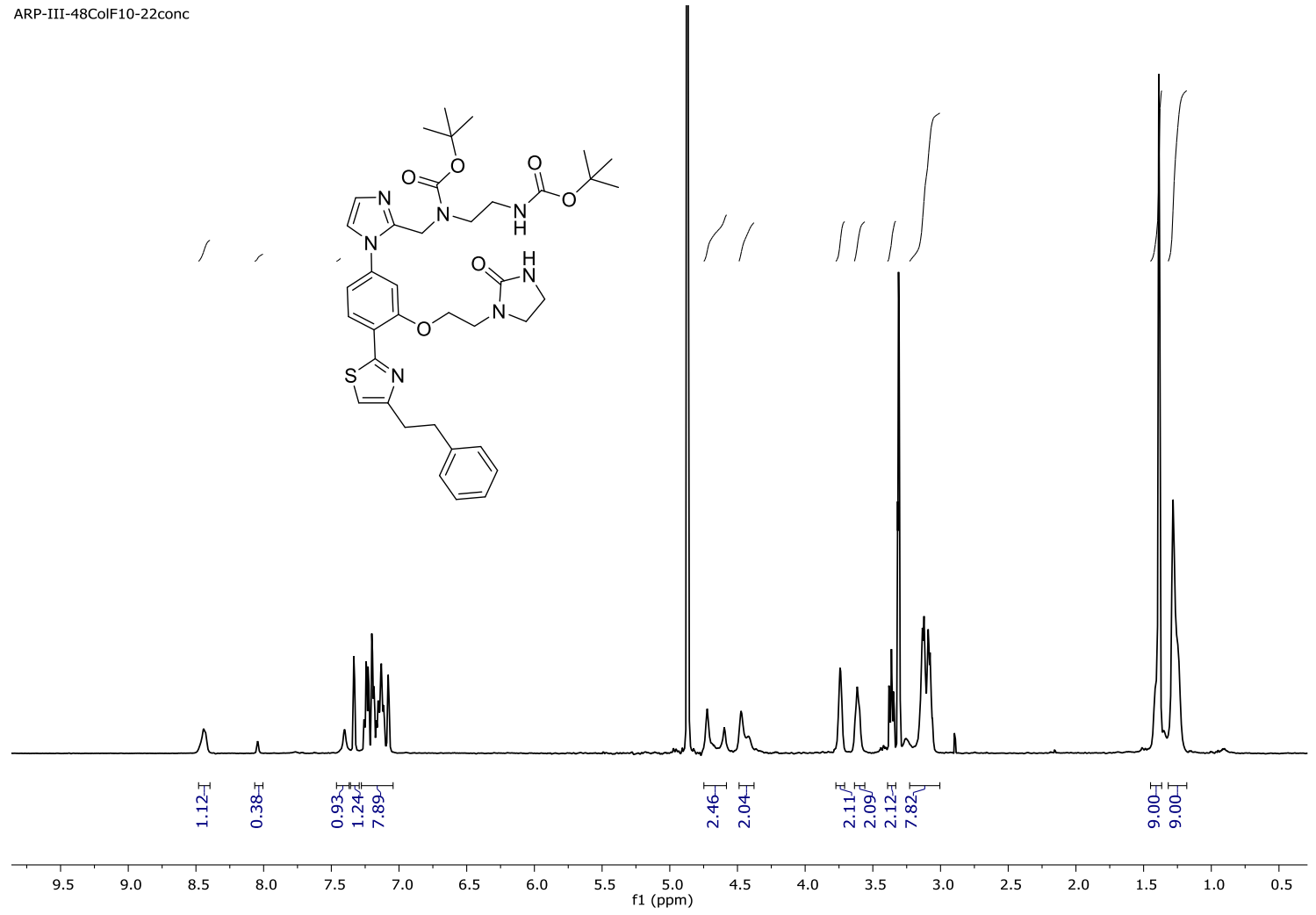




\section{Compound $36 e$}

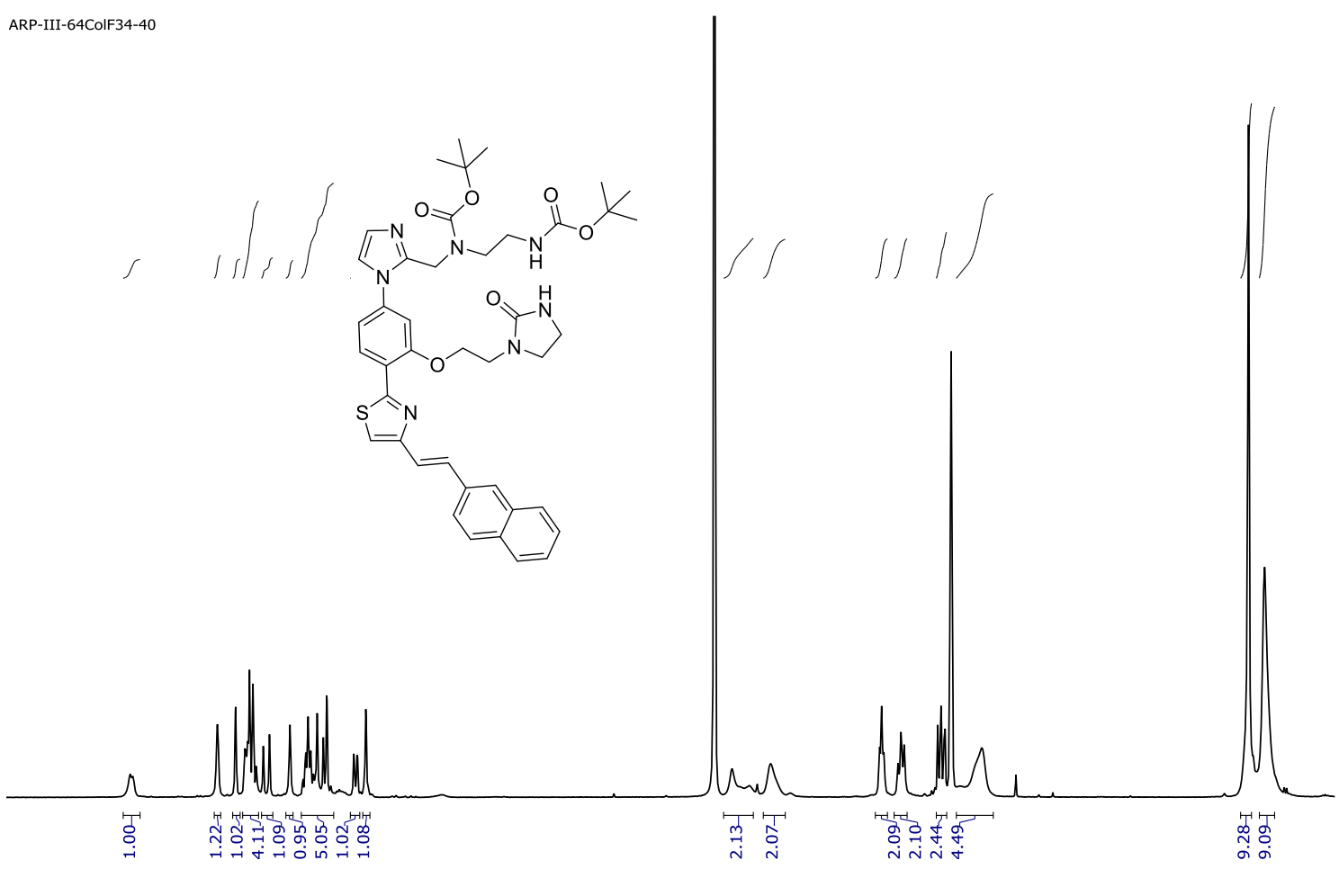

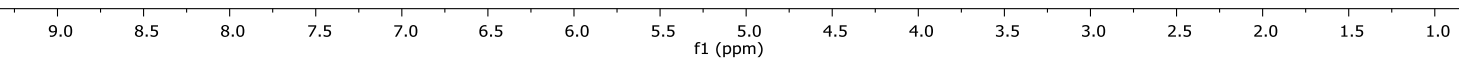

\section{Compound $36 f$}

ARP-VI-31ColF45-50

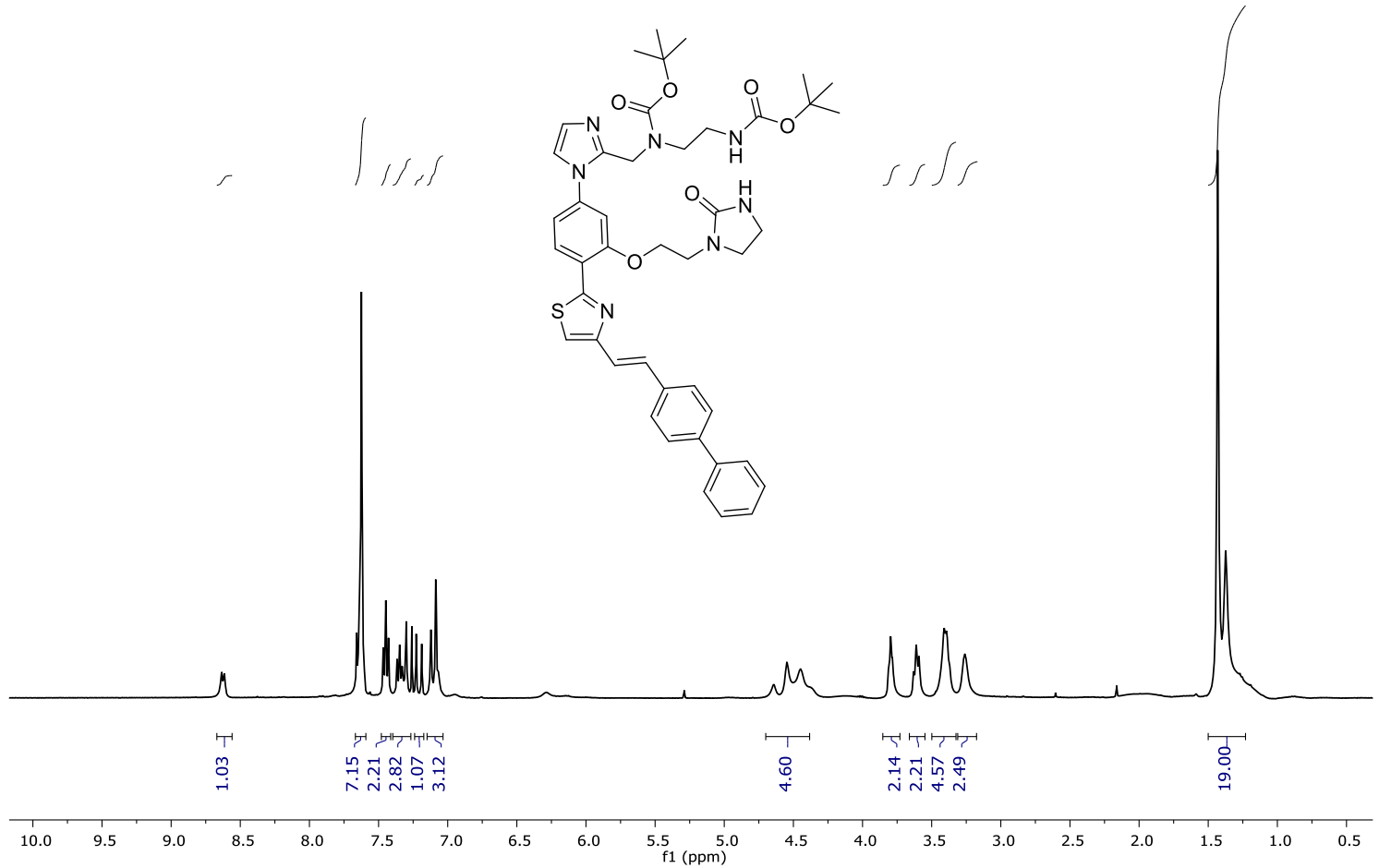




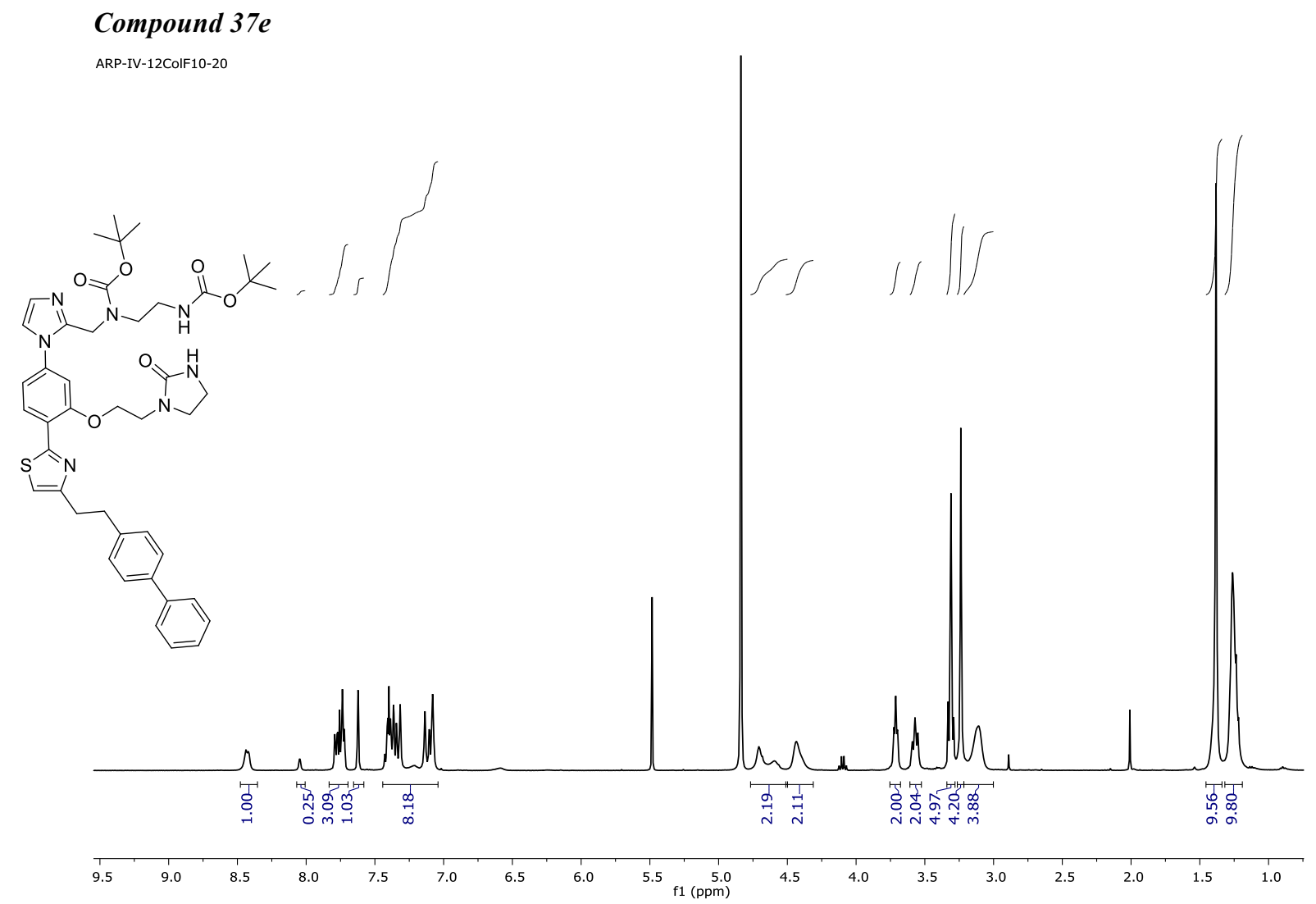

Compound $37 f$

ARP-VI-33ColF10seco

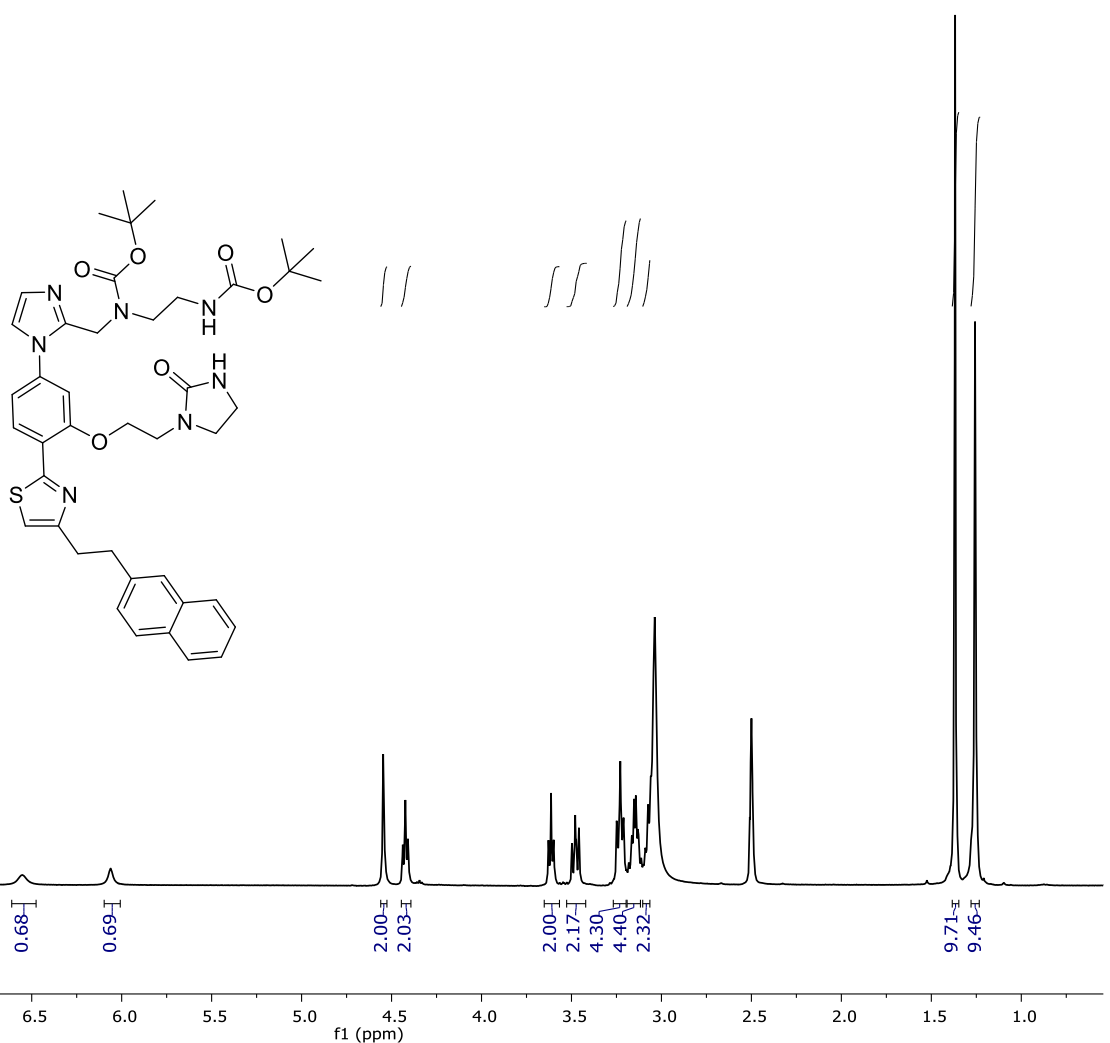




\section{Compound $3 a$}

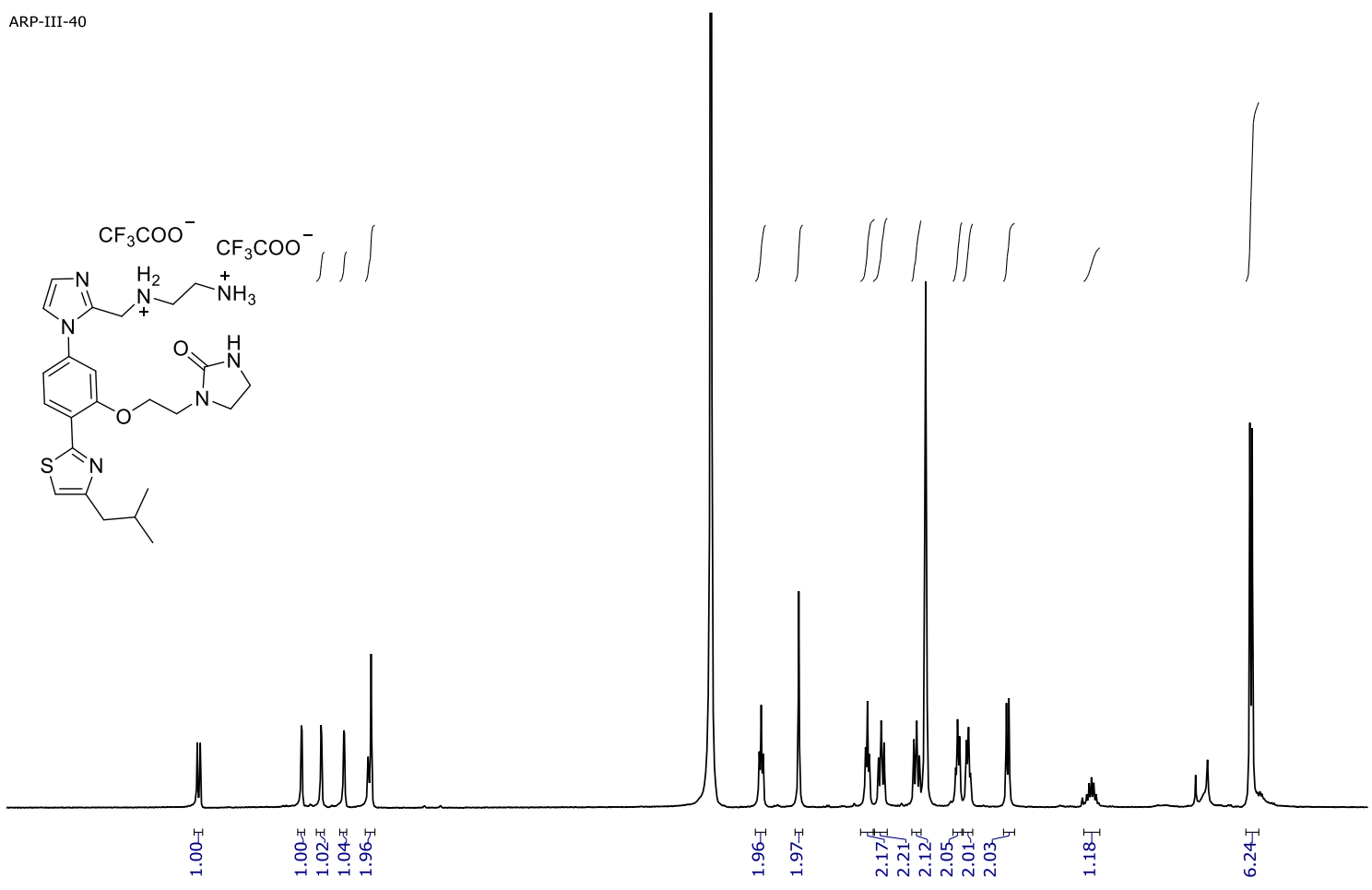

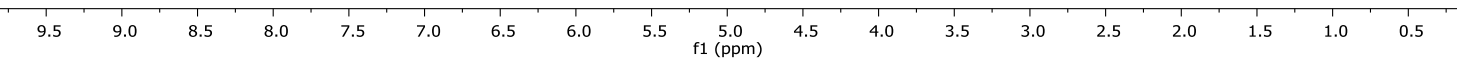

\section{Compound $3 b$}

ARP-III-42

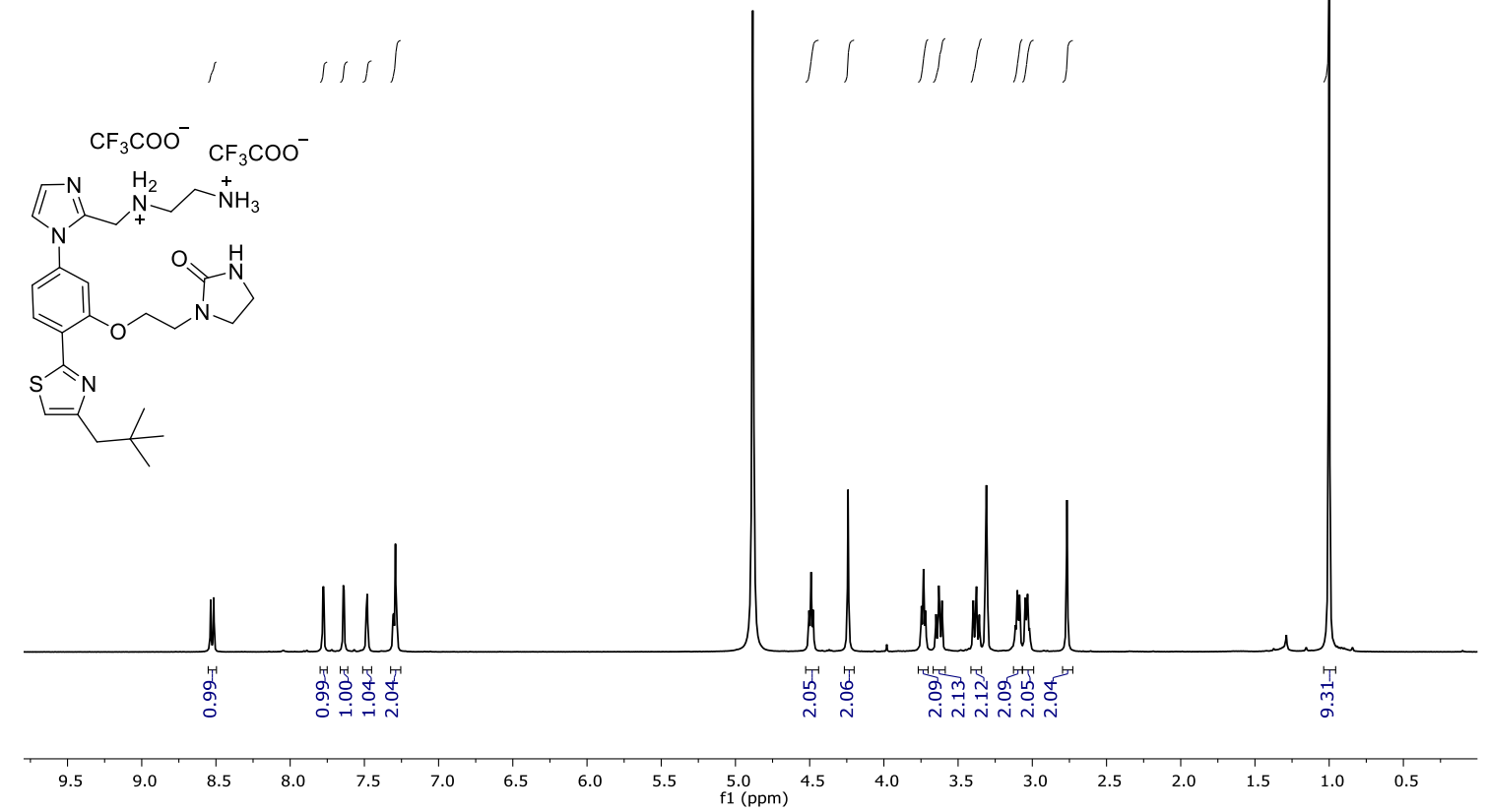


Compound $3 \mathrm{c}$

ARP-III-10CRUDO

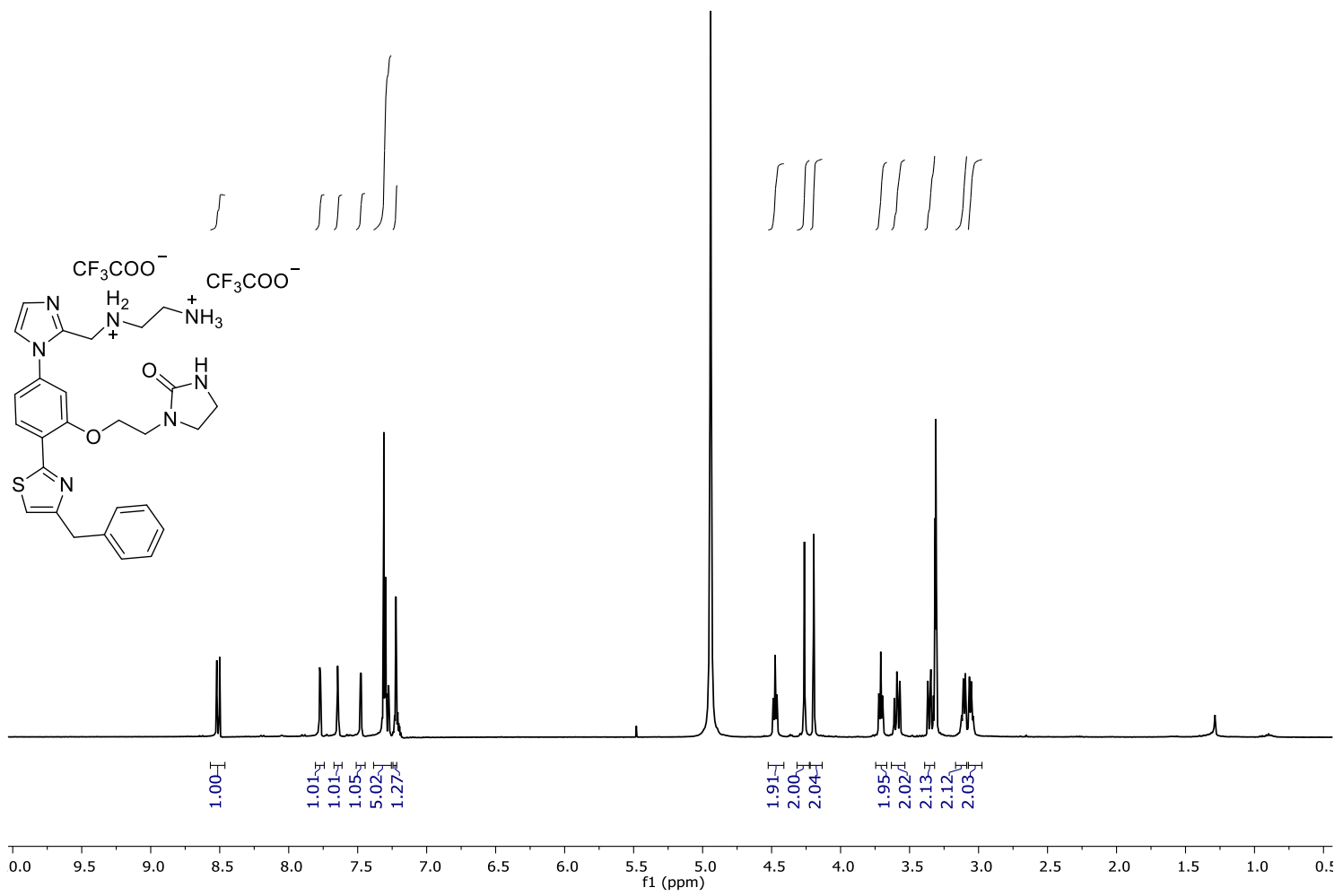

\section{Compound 3d}

ARP-III-50

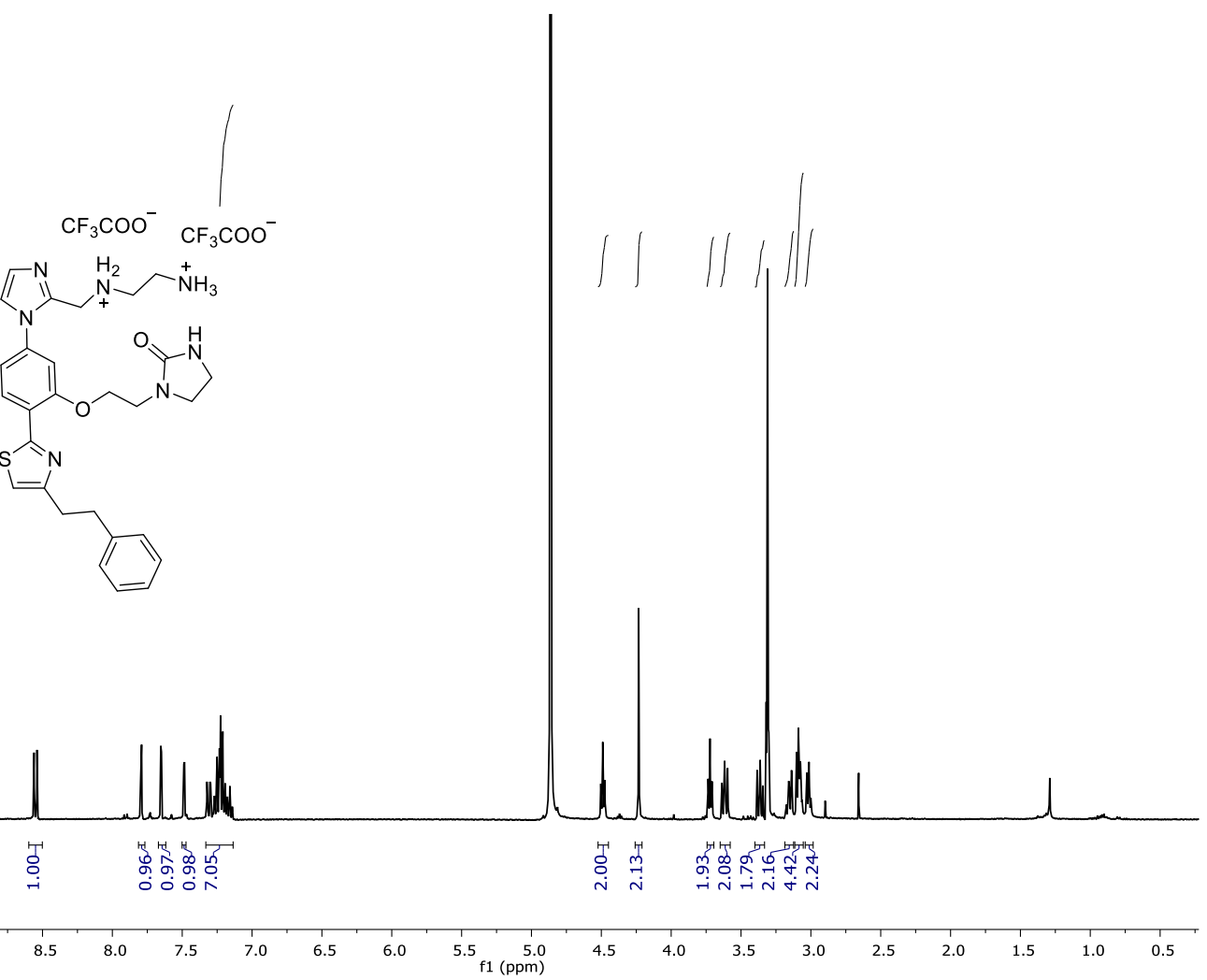




\section{Compound $3 e$}

ARP-IV-17

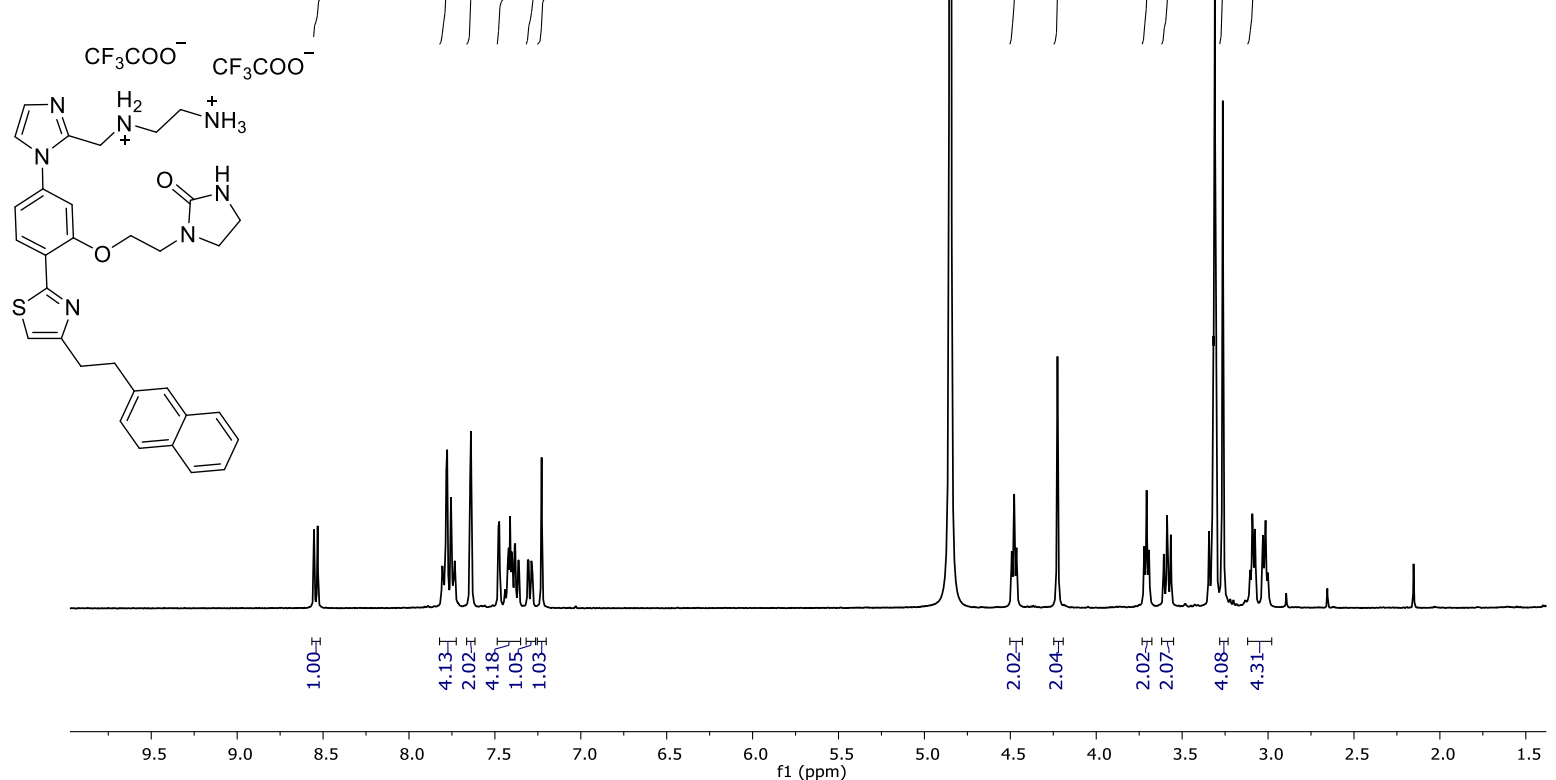

\section{Compound $3 f$}

ARP-VI-39

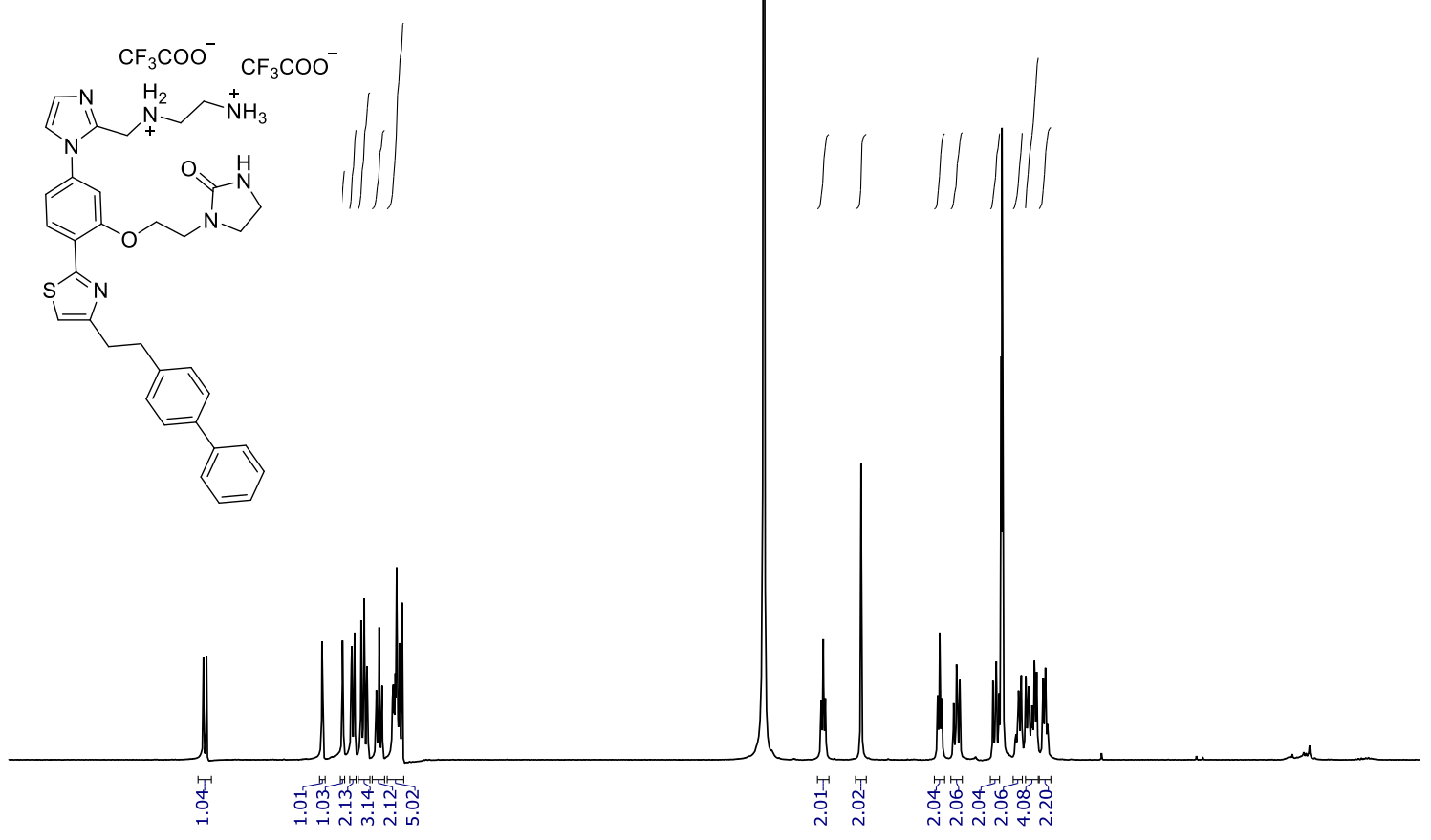

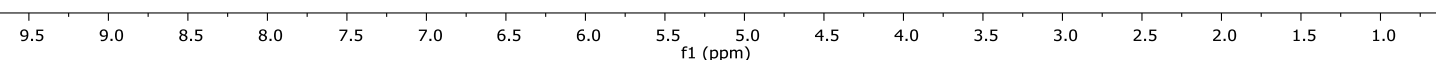


${ }^{13}$ C NMR spectra of Imidazole-phenyl-thiazole 3a-f

Compound 3a

ARP-III-40

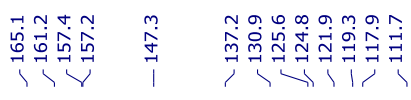
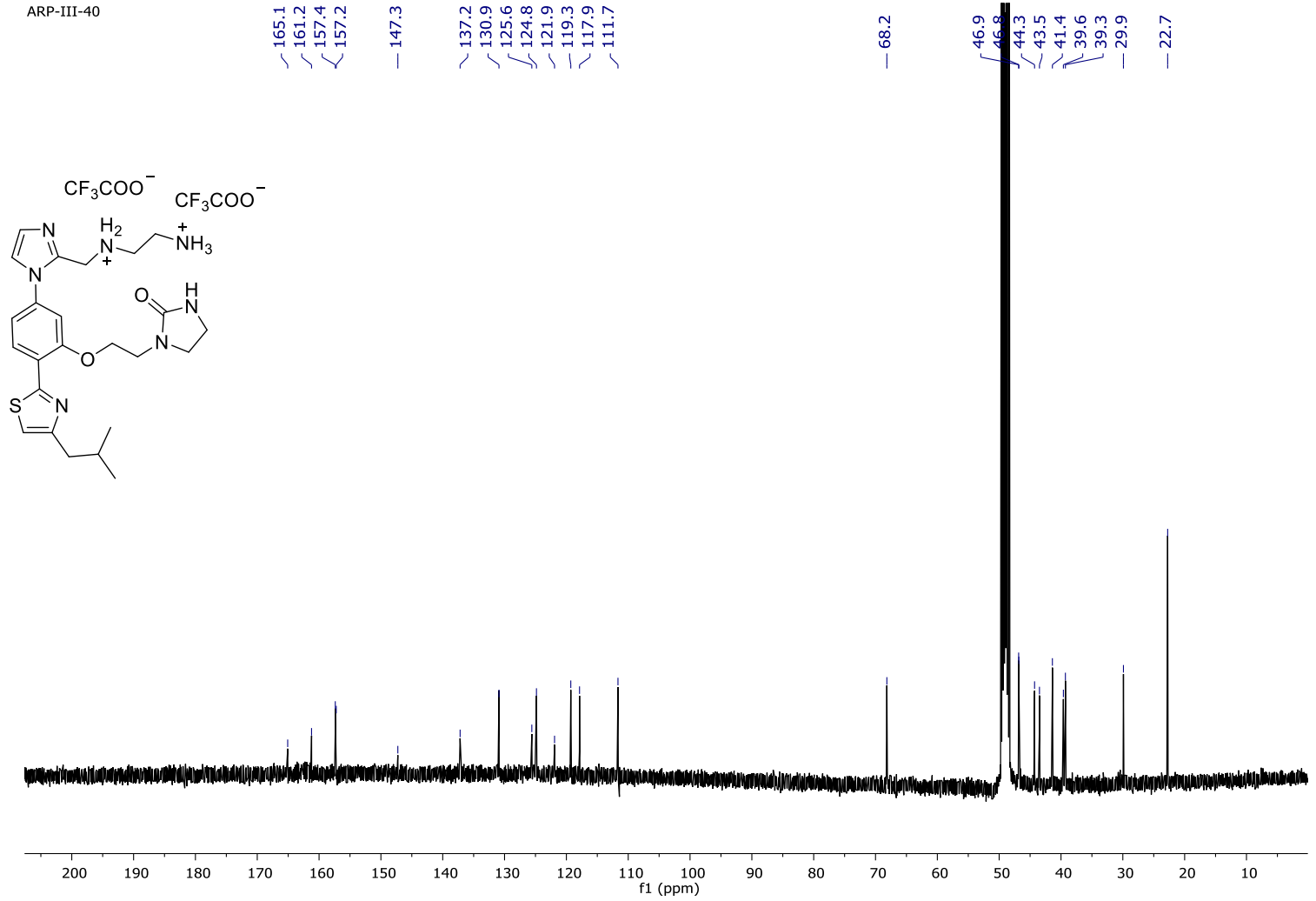

Compound 3b

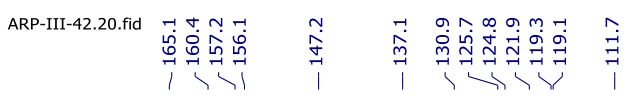
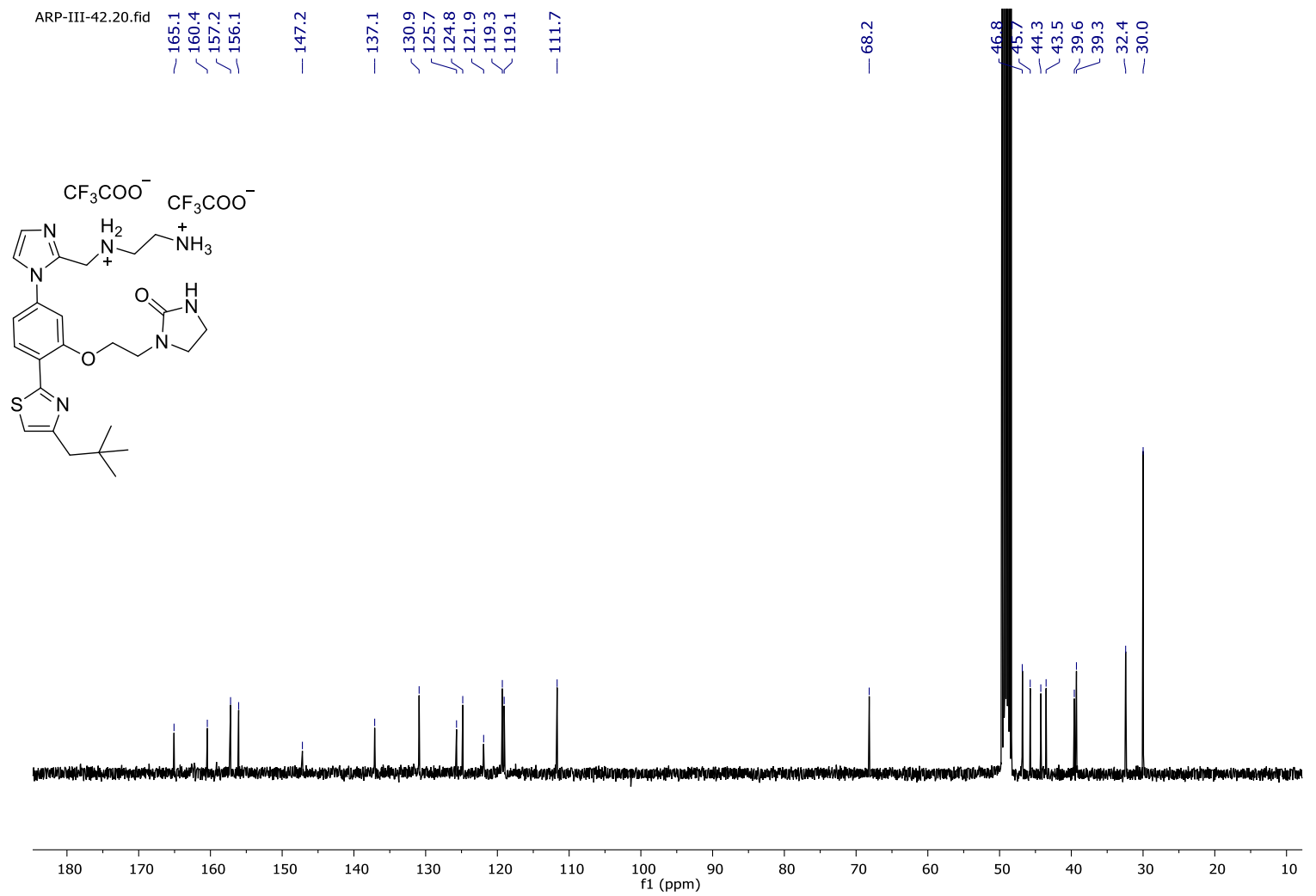

S38 


$$
\begin{array}{ll}
81 \\
\hline 1
\end{array}
$$




\section{Compound $3 e$}

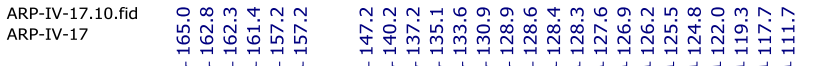

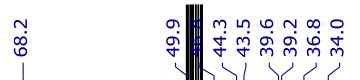

$\mathrm{CF}_{3} \mathrm{COO}^{-} \quad \mathrm{CF}_{3} \mathrm{COO}$

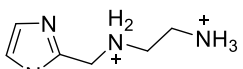

$\overbrace{0}^{2}$

今

$=$

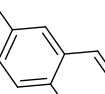

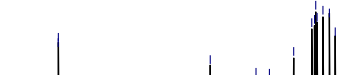

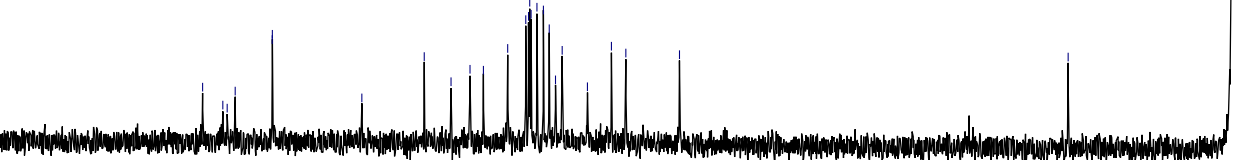

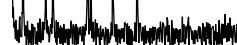

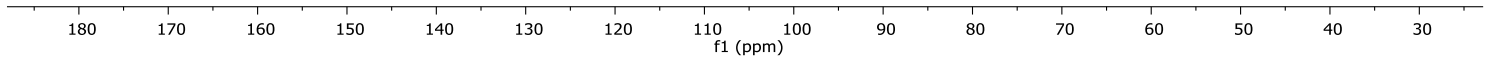

\section{Compound $3 f$}

ARP-VI-39.10.fid تُ
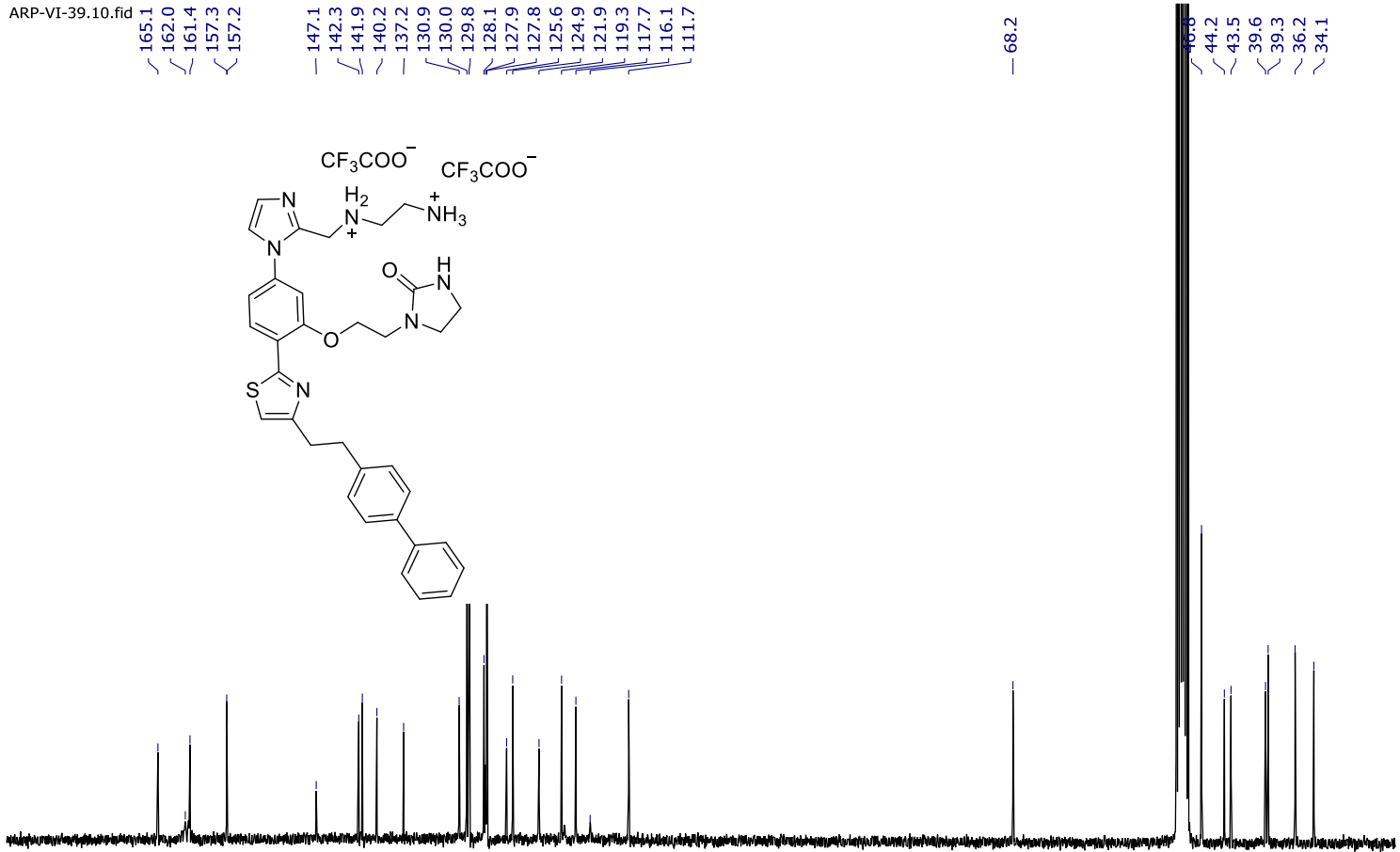

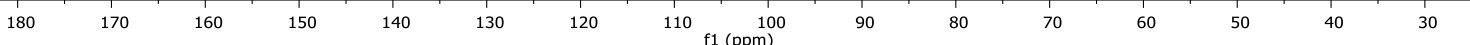

